STATE OF FLORIDA

STATE BOARD OF CONSERVATION

DIVISION OF GEOLOGY

Robert O. Vernon, Director

INFORMATION CIRCULAR NO. 52

WATER LEVELS IN ARTESIAN AND NONARTESIAN AQUIFERS OF FLORIDA, 1963-64

\author{
By \\ Henry G. Healy \\ Prepared by the \\ UNITED STATES GEOLOGICAL SURVEY \\ in cooperation with the \\ DIVISION OF GEOLOGY \\ FLORIDA BOARD OF CONSERVATION \\ and \\ OTHER STATE AND LOCAL AGENCIES
}

TALLAHASSEE

1968 


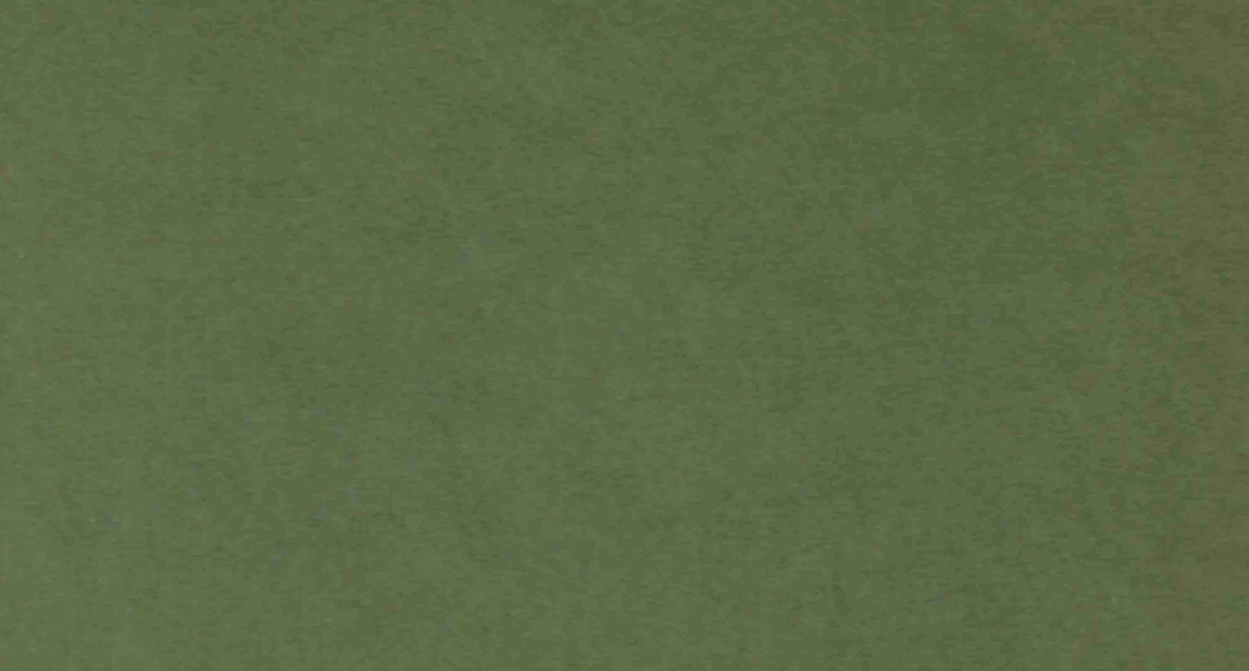

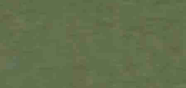

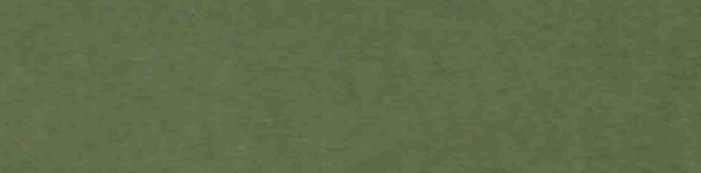

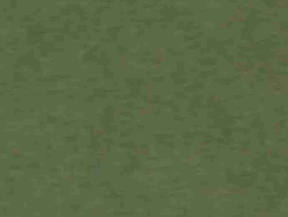

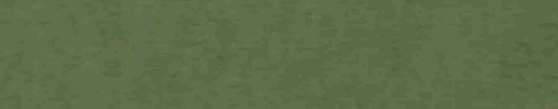

a.

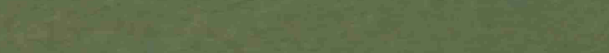

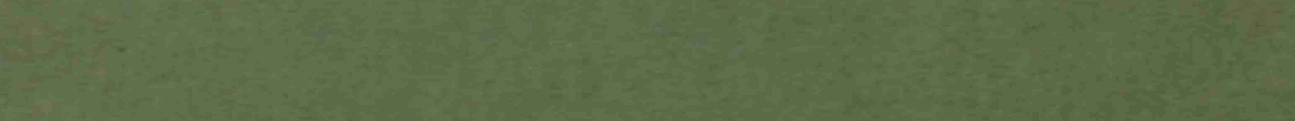
15

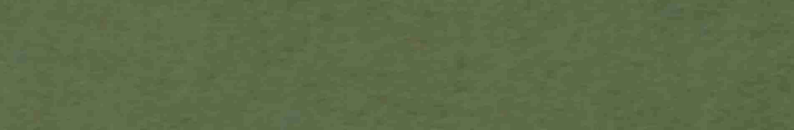

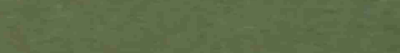

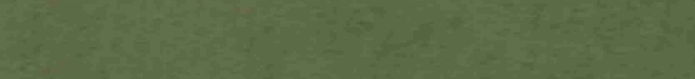


STATE OF FLORIDA

STATE BOARD OF CONSERVATION

\author{
DIVISION OF GEOLOGY \\ Robert O. Vernon, Director
}

INFORMATION CIRCULAR NO. 52

WATER LEVELS IN ARTESIAN AND NONARTESIAN AQUIFERS OF FLORIDA, 1963-64.

\author{
By \\ Henry G. Healy
}

Prepared by the

UNITED STATES GEOLOGICAL SURVEY

in cooperation with the

DIVISION OF GEOLOGY

FLORIDA BOARD OF CONSERVATION

and

OTHER STATE AND LOCAL AGENCIES

TALLAHASSEE

1968 



\section{CONTENTS}

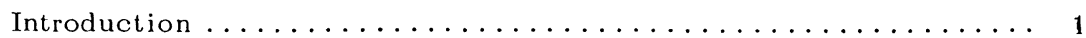

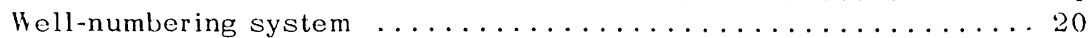

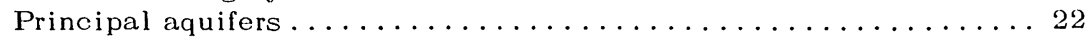

Northwestern Florida ......................... 23

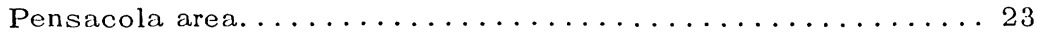

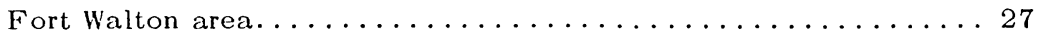

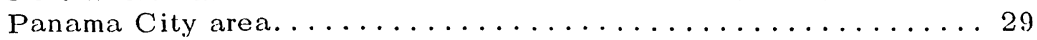

Northern and north-central Florida ................. 29

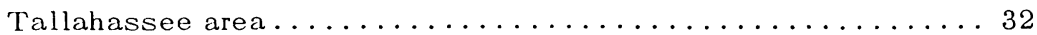

Fernandina-Jacksonville area ................. 35

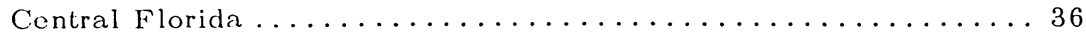

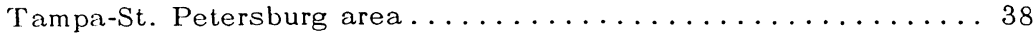

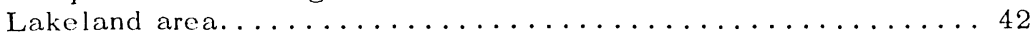

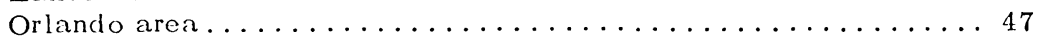

Cape Kennedy area ...................... 51

Sarasota-Bradenton area ................... 52

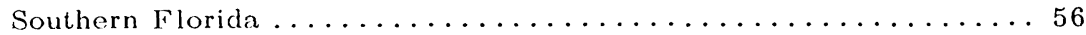

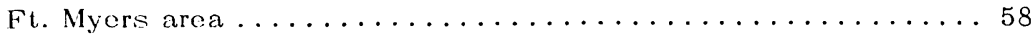

Stuart-licst Palm $\ldots \ldots \ldots$.

Stuart-West Palm Beach area ................ 58

Ft. Lauderdale area. . . . . . . . . . . . . . . . 60

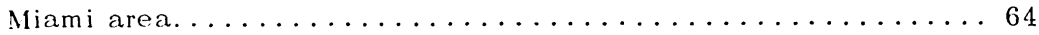

\section{ILLUSTRATIONS}

Figure

1 Observation-well network, December 1964, and the extent of principal aquifers and sources of ground-water supplies

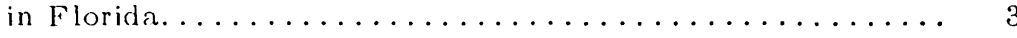

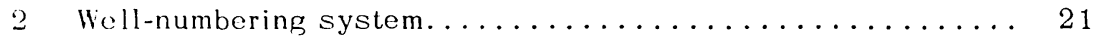

3 Piezometric surface and areas of flow of the Floridan aquifer,

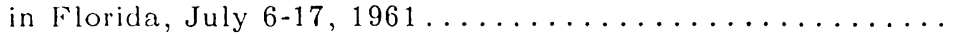

4 Locations of observation wells in northwestern Florida for which hydrographs are given................. 24

$5 \quad$ Graph of total yearly pumpage, City of Pensacola ........ 25

6 Hydrographs showing trends and fluctuations of water levels in wells Escambia 45 at Cantonment, 46 near Ensley, and 62 at Pensacola, Pensacola area................

7 Hydrographs showing trends and fluctuations of water levels in well Escambia 62 and departures from monthly normal precipitation at Pensacola, 1960-64 ..............

8 Hydrographs showing trends and fluctuations of water levels in wells Okaloosa 3,25 , and $31, \mathrm{Ft}$. Walton Beach area.... 28

9 Map showing net change of ground-water levels, Pensacola and Ft. Walton areas, May 1951 to May $1962 \ldots \ldots \ldots \ldots \ldots$

10 Map showing net change of ground-water levels, Pensacola and Ft. Walton areas, May 1962 to May $1964 \ldots \ldots \ldots \ldots \ldots . \ldots 31$

11 Graph of total yearly pumpage, Panama City ........... 32 
12 Hydrographs showing trends and fluctuations of water levels in wells Walton 13 at Point Washington, Bay 7 at Panama

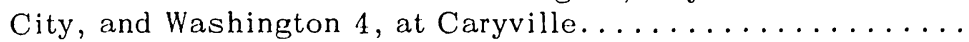

13 Map showing locations of observation wells in northern and north-central Florida for which hydrographs are given ..... 34

14 Graph of total yearly pumpage, City of Tallahassee......... 15 Hydrographs showing trends and fluctuations of water levels in well Leon 7 and departures from monthly normal precipitation at Tallahassee, $1960-64 \ldots \ldots \ldots \ldots \ldots \ldots \ldots \ldots \ldots \ldots \ldots$

16 Hydrographs showing trends and fluctuations of water levels in wells Leon 7 at Tallahassee, Madison 18 near Madison, Columbia 9 at Lake City, and Nassau 12 near Fernandina ...

17 Graph of total yearly pumpage, City of Jacksonville ........

18 Hydrographs showing trends and fluctuations of water levels in wells Nassau 51 at Callahan, Duval 122 at Jacksonville, Duval 164 near Mayport, Marion 5 near Ocala, and Putnam

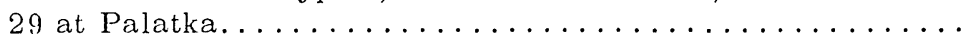

19 Maps showing net changes of ground-water levels in Jacksonville and Fernandina areas, May 1951 to May 1962

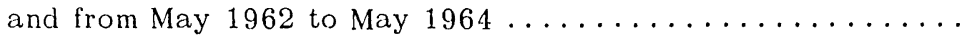

20 Map showing locations of observation wells in central Florida for which hydrographs are given.............

21 Hydrographs showing trends and fluctuations of water levels in wells Pasco 13 near Ehren and Hillsborough 13 near

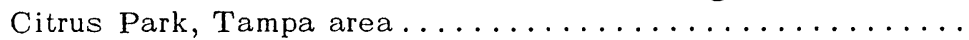

22 Hydrographs showing trends and fluctuations of water levels in well Hillsborough 13 and departures from monthly normal precipitation at Tampa, $1960-64 \ldots \ldots \ldots \ldots \ldots \ldots \ldots$

23 Hydrographs showing trends and fluctuations of water levels in wells Hillsborough 30 near Ruskin, Pinellas 13 at Tarpon Springs, and Pinellas 246 at Clearwater.............

24 Graphs showing changes in chloride content in wells Pinellas 592 at Bay Pines and 166 at Dunedin, St. Petersburg area...

25 Graph showing total yearly pumpage, City of Lakeland...... 26 Hydrographs showing trends and fluctuations of water levels in well Polk 45 near Lakeland and departures from monthly normal precipitation at Lakeland, $1960-64 \ldots \ldots \ldots \ldots \ldots$

27 Hydrographs showing trends and fluctuations of water levels in wells Polk 44 and 47 near Davenport and Polk 45 near

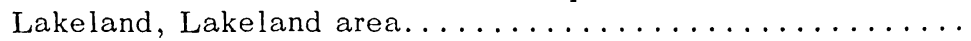

28 Hydrographs showing trends and fluctuations of water levels in wells Polk 49 near Frostproof and Polk 51 at Frostproof,

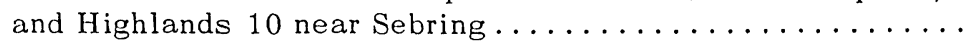

29 Hydrographs showing trends and fluctuations of water levels in wells Highlands 13 , Osceola 183, and Okeechobee 3 in

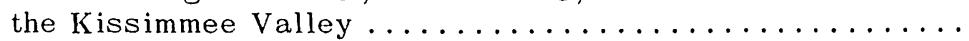

30 Hydrographs showing trends and fluctuations of water levels in wells Orange 47 and $47 \mathrm{~B}$ near Orlando and departures from monthly normal precipitation at Orlando, 1960-64...... 31 Hydrographs showing trends and fluctuations of water levels in well Orange 47 , near Orlando............... 
Hydrographs showing trends and fluctuations of water levels near Cape Kennedy and eastern-central coastal Florida .....

33 Hydrographs showing trends and fluctuations of water levels in wells Manatee 92 and Sarasota 9, Sarasota-Bradenton

34 Map showing location of wells in southern Florida for which

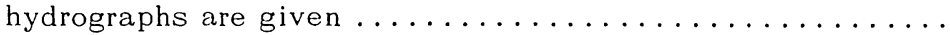

35 Hydrographs showing trends and fluctuations of water levels in well Lee 246 near Ft. Myers and departures from normal monthly precipitation at Ft. Myers, $1960-64 \ldots \ldots \ldots \ldots \ldots$ 36 Hydrographs showing trends and fluctuations of water levels in wells Lee 246 near Ft. Myers, Collier 131 near Immokalee,

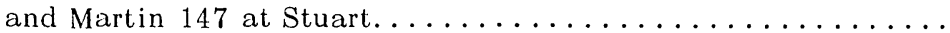

37 Graph of total yearly pumpage, City of Stuart ............ 38 Hydrographs showing trends and fluctuations of water levels in well Martin 147 and departures from monthly normal precipitation at Stuart, $1960-64 \ldots \ldots \ldots \ldots \ldots \ldots \ldots \ldots$

39 Hydrographs showing trends and fluctuations of water levels in well Palm Beach 88 and departures from monthly normal precipitation at West Palm Beach, 1960-64 ........... 40 Hydrographs showing trends and fluctuations of water levels in wells Palm Beach 88 near West Palm Beach, Broward G561 and G617 near Ft. Lauderdale, and Dade G553 near

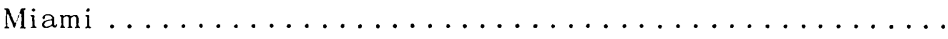

41 Hydrographs showing trends and fluctuations of water levels in wells Broward F291 at Hollywood, Dade S18 near Miami, Dade S196A near Homestead, Dade F179 at Miami, and Broward S329 near Ft. Lauderdale................ 42 Hydrographs showing changes in chloride content of water in wells Broward G5 14 and S830 near Ft. Lauderdale, and

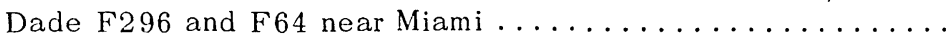
43 Hydrographs showing trends and fluctuations of water levels in well Dade S196A, and departures from monthly normal precipitation at Homestead Experimental Station, 1960-64 ... 44 Hydrographs showing trends and fluctuations of water levels in wells Dade S19 and G10 near Miami, and Dade G72 north-

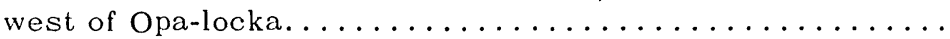
45 Hydrographs showing trends and fluctuations of water levels in wells Dade G596, G618, G613, and G620 in central Dade

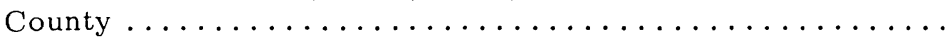
46 Hydrographs showing changes in chloride content of water in wells Dade G354 and G580 near Miami and Dade G469, S529, and G212 in southeastern Dade County.............

Table

1 Well and water-level data for selected observation wells in Florida ............................ 


\title{
WATER LEVELS IN ARTESIAN ANU NONARTESIAN AQUIFERS OF FLORIDA, 1963-64
}

\author{
By \\ Henry G. Healy \\ INTRODUCTION
}

This report summarizes the trends and fluctuations of groundwater levels in the principal aquifers in Florida during 1963-64 and includes the following: (1) hydrographs of ground-water levels in the several aquifers; (2) maps showing changes in ground-water levels during specific periods; and, (3) a table summarizing the principal data on selected observation wells. Observation wells for which records are available are listed in the "Index to Water Resources Data-Collection Stations in Florida, 1961", Florida Geological Survey Special Publication No. 11, which includes the location, aquifer, and type and period of records available for about 3,600 observation wells.

Since World War II, and particularly during the last decade, the demand for fresh water for industrial, municipal, and agricultural use in Florida has increased yearly. Although ground-water supplies have been adequate for the increased demand in most areas in Florida, water levels have declined appreciably in some areas. Because demand for ground water continues to increase, shortages will occur and may become critical in some areas. In coastal areas, declining water levels may allow salt water to encroach and shortages could result from deterioration in quality as well as from the reduction of quantity of water available. In order to prevent future shortages, the supplies of ground water must be properly appraised before they can be effectively utilized. Records of trends and fluctuations of ground-water levels have long formed a basis for such an appraisal.

The principal objective of the Water Resources Division of the U.S. Geological Survey is to appraise and to evaluate the Nation's water resources. Although many types of ground-water investigations are conducted throughout the Nation, the collection and compilation of basic hydrologic data constitute an important part of the water-resources studies.

Objectives of the hydrologic data program include: the evaluation of available ground-water supplies; the prediction of trends 
of water levels; and the delineation of present or potential areas of detrimentally high or low ground-water levels. Water levels are also used to predict the base flow of streams, to portray the effects of natural and man-induced forces that act on a water-bearing formation, and to furnish information for use in research. The hydrologic data program is an important adjunct of the several types of geologic and hydrologic methods of study used in waterresources investigations.

The hydrologic data-collection program of the U. S. Geological Survey is part of the cooperative investigations of the ground-water resources of Florida, in cooperation with the Division of Geology, Florida Board of Conservation, and other state and local agencies and municipalities. The observation-well network in 1964 included about 1,000 observation wells in the 67 counties of the State. Figure 1 shows the locations of these observation wells and Table 1 lists data for 329 observation wells selected from the statewide network.

The hydrologic-data program consists of the collection, tabulation, interpretation, evaluation, and publication of water-level and related data. Water levels for selected wells are published, at present, once every 5 years in the U. S. Geological Survey Water-Supply Papers.

Information pertinent to ground water is also published in interpretative reports of investigations published by the Florida Geological Survey and the U. S. Geological Survey. Data collected during an investigation and subsequent to publication are available from the District Chief; U. S. Geological Survey, P. O. Box 2315, Tallahassee, Florida 32304.

The water-level data used in this report represent measurements taken from automatic water-stage recorder charts, pressure gages, and made by wet tape. In general, water-level measurements made by tape and stage recorder are shown to the nearest hundredth of a foot, and those made with a pressure gage are shown to the nearest tenth of a foot.

Table 1 summarizes well-data and water-level information for the several aquifers. Well data include the aquifer, depth of well, and depth of casing. The items: "Year record began" and "Frequency of measurements" are included as part of the well data. In the water-level portion of the table, levels for May or June are used because records are available for these months for a large percentage of the wells. Also, ground-water levels generally are lowest during May or June in many areas and records during that 
UNITED STATES DEPARTMENT OF THE INTERIOR

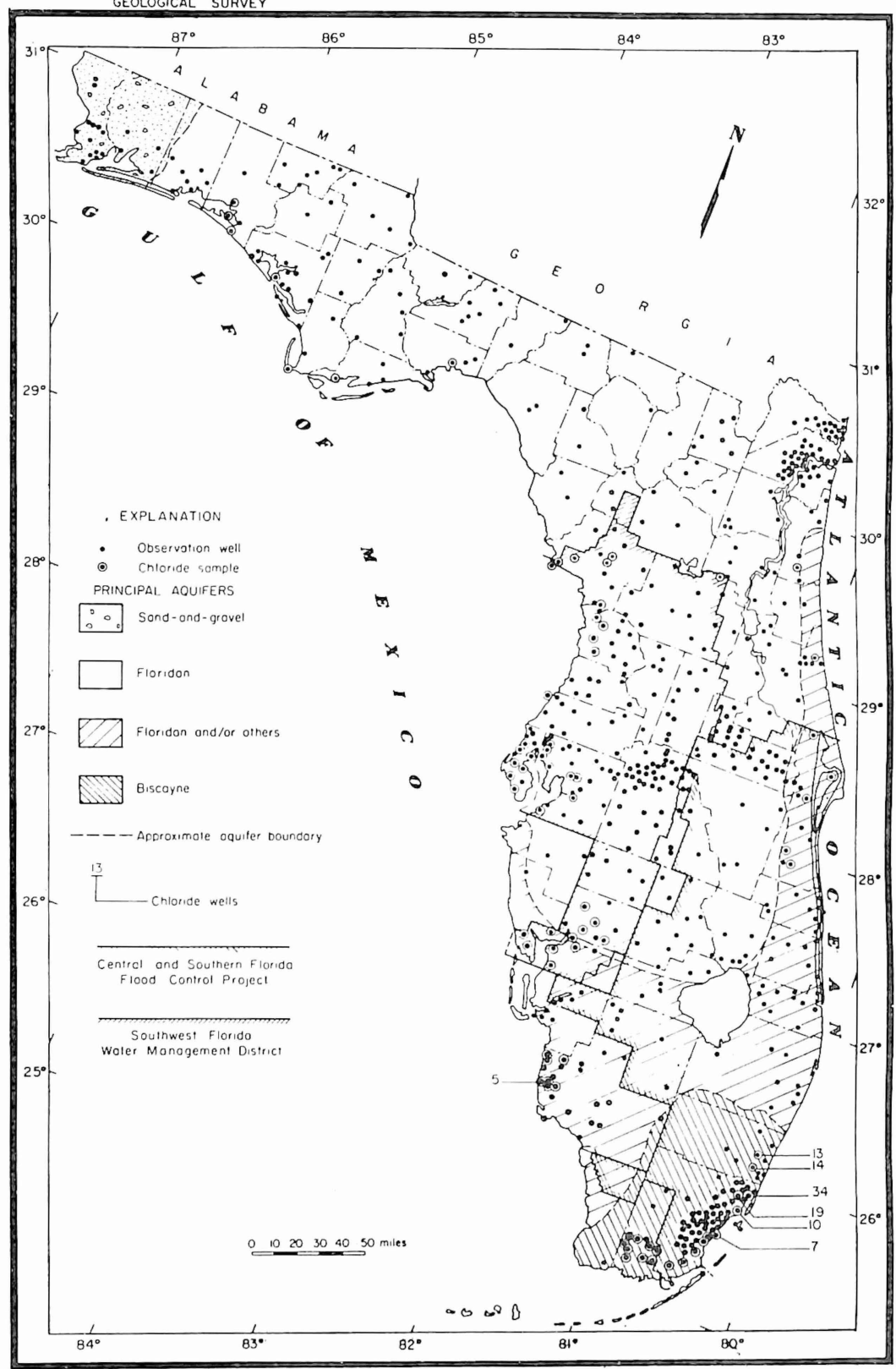

Figure 1. Observation-well network, December 1964, and the extent of principal aquifers and sources of ground-water supplies in Florida. 
Table 1.--Sumary of well data and water levelo in selected observation wells.

Well number: Well numbera are based on county numbering system e.g. Bay County well Bay 20 , or on the latitudinal and longitudinal system c.g. well 008-537-2. Both numbers 20 and 008-537-2 are given where this well has been reported previously in a publication under the county number. Letters preflxed to well numbers in Broward and Dade Counties; G, Geological Survey wells,; S, supply wells; F, fire wells; and NP, National Park Service wells. Letter suffix A, shallow well adjacent to deep well.

Aquifer: B, Biscayne; F, Floridan; G, sand-and-gravel; H, Hawthorn; NA, nonartesian; S, shallow sand.

Depth of well: Measured unless otherwise noted. $R$, reported depth.

Frequency of tneasurement: Refers to current biennium. B, bimonthly; C, continuous; M, monthly; S, semiannually; $W$, weekly.

Prior to 1963: When only one measurement is avallable prior to current biennium, measurement is arbitrarily listed as a low level.

Water level: To hundredth of a foot if measured by wet-tape method or taken from recorder chart; to nearest tenth of a foot if measured by pressure gage or airlíne.

Annual range: Based on measurements available during year. For wells equipped with recorder, range is based on every 5 th day measurements.

Remarks: $B$, water level below measuring point; $D$, measurements discontinued on date shown in Remarks; $L$, lowest water level; $M$, water level with reference to mean sea level; $P$, water level affected by pumping of ncarby wells; $R$, recorder installed on date shown in Remarks; $S$, water level affected by seasonal or regional punping; $T$, water levels affected by ocean tides.

\begin{tabular}{|c|c|c|c|c|c|c|c|c|c|c|c|c|}
\hline \multirow{4}{*}{ We 11 number } & \multirow{4}{*}{ 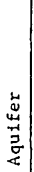 } & \multirow{4}{*}{ 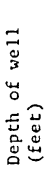 } & \multirow{4}{*}{ 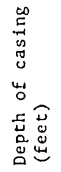 } & \multirow{4}{*}{ 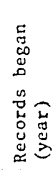 } & \multirow{4}{*}{ 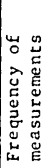 } & \multicolumn{6}{|c|}{$\begin{array}{c}\text { Water level above }(+) \text { or below }(-) \text { land surface } \\
\text { (feet) }\end{array}$} & \multirow{4}{*}{ Remarks } \\
\hline & & & & & & \multicolumn{2}{|c|}{ Prior to 1963} & \multirow{2}{*}{\multicolumn{2}{|c|}{$\begin{array}{l}\text { Highest water } \\
\text { level in May } \\
\text { or June }\end{array}$}} & \multirow{2}{*}{\multicolumn{2}{|c|}{$\begin{array}{l}\text { Annual } \\
\text { Range }\end{array}$}} & \\
\hline & & & & & & \multicolumn{2}{|c|}{ May or June } & & & & & \\
\hline & & & & & & $\begin{array}{l}\text { High } \\
\text { (year) }\end{array}$ & $\begin{array}{l}\text { Low } \\
\text { (year) }\end{array}$ & 1963 & 1964 & 1963 & 1964 & \\
\hline
\end{tabular}

ALACHUA COUNTY

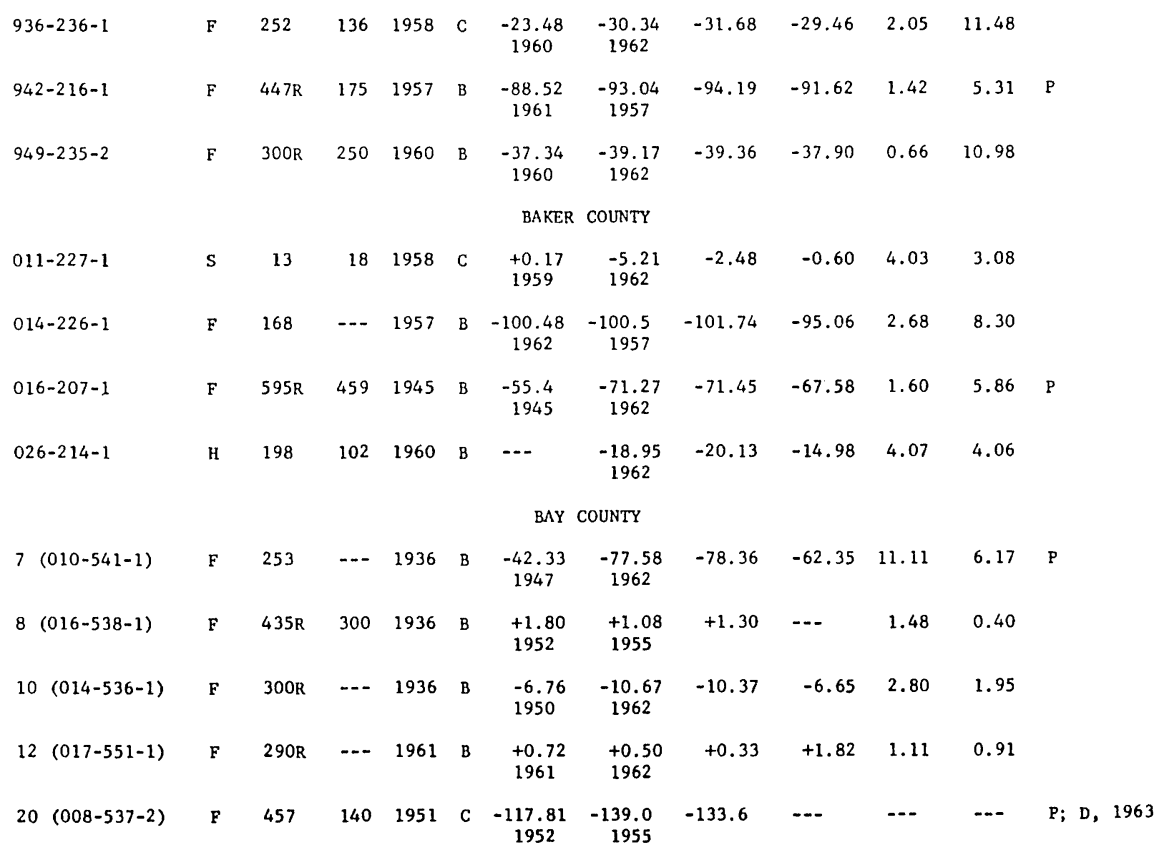




\begin{tabular}{|c|c|c|c|c|c|c|c|c|c|c|c|c|}
\hline \multirow{4}{*}{ Well number } & \multirow{4}{*}{ 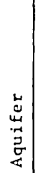 } & \multirow{4}{*}{ 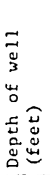 } & \multirow{4}{*}{ 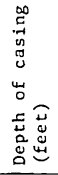 } & \multirow{4}{*}{ 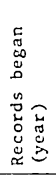 } & \multirow{4}{*}{ 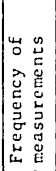 } & \multicolumn{6}{|c|}{$\begin{array}{c}\text { Water level above }(+) \text { or below }(-) \text { land surface } \\
\text { (fect) }\end{array}$} & \multirow{4}{*}{ Remarks } \\
\hline & & & & & & \multirow{2}{*}{\multicolumn{4}{|c|}{\begin{tabular}{|c|c|} 
Prior to 1963 & $\begin{array}{c}\text { Highest water } \\
\text { level in May } \\
\text { or June or June }\end{array}$ \\
\end{tabular}}} & \multirow{2}{*}{\multicolumn{2}{|c|}{$\begin{array}{l}\text { Annual } \\
\text { Range }\end{array}$}} & \\
\hline & & & & & & & & & & & & \\
\hline & & & & & & $\begin{array}{l}\text { High } \\
\text { (year) }\end{array}$ & $\begin{array}{l}\text { Low } \\
\text { (year) }\end{array}$ & 1963 & 1964 & 1963 & 1964 & \\
\hline
\end{tabular}

\begin{tabular}{|c|c|c|c|c|c|c|c|c|c|c|c|c|c|}
\hline 43 & $(004-535-1)$ & $\mathrm{F}$ & 645 & 238 & 1946 & B & $\begin{array}{r}-62.8 \\
1948\end{array}$ & $\begin{array}{r}-128.7 \\
1962\end{array}$ & -102.9 & -82.2 & 37.0 & $\cdots$ & $\mathbf{P}$ \\
\hline 53 & $(012-552-1)$ & G & 134 & 114 & 1961 & B & -- & $\begin{array}{l}-8.97 \\
1962\end{array}$ & -9.32 & -8.37 & 0.51 & -- & D, 1964 \\
\hline 65 & $(006-525-1)$ & $F$ & $200 \mathrm{R}$ & $\cdots$ & 1961 & B & $\cdots$ & $\begin{array}{l}+4.30 \\
1962\end{array}$ & +2.3 & --- & 4.0 & $\cdots$ & D, 1964 \\
\hline 68 & $(023-526-1)$ & $\mathrm{F}$ & 160 & 158 & 1961 & B & $\cdots$ & $\begin{array}{l}+1.81 \\
1962\end{array}$ & +1.6 & +3.30 & 1.57 & 1.90 & \\
\hline \multirow[t]{2}{*}{69} & $(025-525-1)$ & G & 153 & 136 & 1961 & B & $\cdots$ & $\begin{array}{c}-13.76 \\
1962\end{array}$ & -13.14 & -8.25 & 3.26 & -- & D, 1964 \\
\hline & & & & & & & BRADFORD & COUNTY & & & & & \\
\hline \multirow[t]{2}{*}{000} & $-210-2$ & $\mathbf{F}$ & 294 & 247 & 1959 & B & $\begin{array}{c}-69.52 \\
1959\end{array}$ & $\begin{array}{c}-73.96 \\
1962\end{array}$ & -75.69 & -73.47 & 1.29 & 4.95 & \\
\hline & & & & & & & BREVARD & COUNTY & & & & & \\
\hline 19 & $(805-045-1)$ & $\mathbf{F}$ & $413 k$ & 80 & 1934 & B & $\begin{array}{r}+27.3 \\
1950\end{array}$ & $\begin{array}{r}+19.6 \\
1961\end{array}$ & +15.2 & +21.6 & 8.7 & 4.2 & P; D, 1964 \\
\hline 20 & $(795-043-2)$ & $\mathbf{F}$ & $447 R$ & 125 & 1934 & B & $\begin{array}{r}+28.7 \\
1947\end{array}$ & $\begin{array}{r}+19.8 \\
1962\end{array}$ & +20.0 & +20.6 & 3.7 & 3.1 & $\mathrm{~S}$ \\
\hline 79 & $(847-051-1)$ & $\mathbf{F}$ & $160 \mathrm{R}$ & 85 & 1946 & B & $\begin{array}{l}+5.1 \\
1947\end{array}$ & $\begin{array}{l}-0,55 \\
1962\end{array}$ & -- & +3.12 & 1.78 & 1.56 & $\mathrm{~S}$ \\
\hline 148 & $3(821-045-1)$ & $\mathbf{F}$ & $206 R$ & 105 & 1946 & B & $\begin{array}{r}+10.9 \\
1953\end{array}$ & $\begin{array}{l}+4.3 \\
1962\end{array}$ & +5.04 & +7.1 & 3.62 & 2.3 & $\mathrm{~S}$ \\
\hline 159 & $(834-039-1)$ & $\mathbf{F}$ & 210 & 144 & 1946 & B & $\begin{array}{r}+14.9 \\
1953\end{array}$ & $\begin{array}{l}+8.2 \\
1958\end{array}$ & +9.2 & -- & 3.8 & 0.6 & S; D, 1964 \\
\hline \multicolumn{2}{|c|}{$759-045-1$} & $\mathrm{~S}$ & 9 & 4 & 1958 & $\mathrm{C}$ & $\begin{array}{l}-4.4 \\
1958\end{array}$ & $\begin{array}{l}-7.2 \\
1962\end{array}$ & -6.2 & -3.5 & 3.2 & 2.8 & \\
\hline \multicolumn{2}{|c|}{$807-039-2$} & $\mathrm{~S}$ & 50 & 4 & 1958 & C & $\begin{array}{l}-6.5 \\
1959\end{array}$ & $\begin{array}{l}-8.4 \\
1962\end{array}$ & -7.6 & -6.1 & 3.4 & 2.3 & \\
\hline \multicolumn{2}{|c|}{$814-048-2$} & $\mathrm{~S}$ & 9 & 4 & 1958 & C & $\begin{array}{l}-0.9 \\
1959\end{array}$ & $\begin{array}{l}-3.1 \\
1961\end{array}$ & -2.1 & 0.0 & 2.8 & 3.1 & \\
\hline \multirow{2}{*}{\multicolumn{2}{|c|}{$822-047-2$}} & $\mathrm{~F}$ & 129 & 4 & 1960 & $\mathrm{C}$ & $\begin{array}{r}+32.6 \\
1960\end{array}$ & $\begin{array}{r}+29.9 \\
1960\end{array}$ & +27.9 & +30.2 & 4.5 & 3.60 & M \\
\hline & & & & & & & BROWARD & COUNTY & & & & & \\
\hline \multicolumn{2}{|c|}{ P291 } & B & 107 & -- & 1939 & $\mathrm{C}$ & $\begin{array}{l}+4.3 \\
1958\end{array}$ & $\begin{array}{l}+0.4 \\
1952\end{array}$ & +1.61 & +2.90 & 4.09 & 2.76 & $M$ \\
\hline \multicolumn{2}{|c|}{ G561 } & B & 20 & 20 & 1948 & C & $\begin{array}{l}+4.1 \\
1958\end{array}$ & $\begin{array}{l}+0.2 \\
1956\end{array}$ & +2.05 & +2.97 & 4.40 & 3.45 & $M$ \\
\hline \multicolumn{2}{|c|}{ G616 } & B & 25 & 19 & 1952 & C & $\begin{array}{l}+12.90 \\
1957 \& 58\end{array}$ & $\begin{array}{l}+8.72 \\
1956\end{array}$ & +11.66 & +11.28 & 4.06 & 3.36 & $\mathbf{M}$ \\
\hline \multicolumn{2}{|c|}{6617} & B & 29 & 28 & 1950 & C & $\begin{array}{l}+6.6 \\
1954\end{array}$ & $\begin{array}{l}+2.57 \\
1962\end{array}$ & +4.54 & +5.96 & 2.13 & 3.40 & $M$ \\
\hline \multicolumn{2}{|c|}{$\operatorname{cs20}$} & B & 224 & 215 & 1956 & $\mathrm{C}$ & $\cdots$ & $\begin{array}{l}-0.70 \\
1962\end{array}$ & -0.70 & +1.15 & 7.45 & 5.87 & $\begin{array}{l}\text { M; Prospect } \\
\text { we } 11 \text { field }\end{array}$ \\
\hline \multicolumn{2}{|c|}{$\cos 3$} & B & 22 & 21 & 1960 & C & $\cdots$ & $\begin{array}{l}+2.80 \\
1962\end{array}$ & +3.40 & +3.75 & 5.82 & 4.15 & $\begin{array}{l}\text { M; Poopano } \\
\text { well fleld }\end{array}$ \\
\hline \multicolumn{2}{|c|}{8329} & B & 68 & -- & 1940 & C & $\begin{array}{l}+5.5 \\
1955\end{array}$ & $\begin{array}{l}+0.5 \\
1954\end{array}$ & +1.59 & +2.58 & 4.48 & 5.3 & $\begin{array}{l}\text { M; D1xic well } \\
\text { fleld }\end{array}$ \\
\hline
\end{tabular}




\begin{tabular}{|c|c|c|c|c|c|c|c|c|c|c|c|c|}
\hline \multicolumn{13}{|c|}{ CALHOUN COUNTY } \\
\hline $1(026-502-1)$ & $\mathrm{F}$ & 212 & 36 & 1961 & B & $\begin{array}{l}-2.27 \\
1961\end{array}$ & $\begin{array}{l}-3.05 \\
1962\end{array}$ & -3.00 & -0.43 & 2.40 & 1.92 & \\
\hline $7(026-509-1)$ & $\mathrm{F}$ & $188 \mathrm{R}$ & 64 & 1961 & B & $\begin{array}{l}+8.0 \\
1961\end{array}$ & $\begin{array}{l}+7.4 \\
1962\end{array}$ & +7.4 & +10.6 & 1.6 & 2.0 & \\
\hline $11(014-511-1)$ & $\mathbf{F}$ & $147 R$ & 47 & 1961 & B & $\begin{array}{r}+11.7 \\
1961\end{array}$ & $\begin{array}{r}+10.9 \\
1962\end{array}$ & +10.8 & +13.6 & 3.1 & 1.8 & \\
\hline \multicolumn{13}{|c|}{ CITRUS COUNTY } \\
\hline $15(902-228-1)$ & $F$ & 78 & -- & 1933 & B & $\begin{array}{l}-8.62 \\
1959\end{array}$ & $\begin{array}{c}-19.83 \\
1956\end{array}$ & -19.87 & -15.01 & 2.00 & 10.57 & \\
\hline $856-223-2$ & $\mathbf{F}$ & 91 & $\cdots$ & 1961 & B & $\cdots$ & $\begin{array}{c}-48.36 \\
1962\end{array}$ & -48.58 & -45.38 & 1.79 & 14.05 & \\
\hline \multicolumn{13}{|c|}{ CLAY COUNTY } \\
\hline $5(006-148-2)$ & $\mathrm{F}$ & $530 R$ & 157 & 1940 & B & $\begin{array}{r}+35.5 \\
1947\end{array}$ & $\begin{array}{r}+21.0 \\
1957\end{array}$ & +21.3 & +26.1 & 3.0 & 2.4 & \\
\hline $948-202-6$ & $\mathrm{H}$ & 144 & 80 & 1960 & B & $\begin{array}{c}-45.33 \\
1960\end{array}$ & $\begin{array}{c}-47.72 \\
1962\end{array}$ & -51.06 & -49.43 & 2.00 & 4.40 & \\
\hline $948-202-7$ & NA & 42 & 40 & 1960 & B & $\begin{array}{c}-28.38 \\
1960\end{array}$ & $\begin{array}{c}-30.94 \\
1962\end{array}$ & -35.70 & -31.53 & $1: 82$ & 6.12 & \\
\hline $948-202-8$ & $\mathbf{F}$ & 250 & 193 & 1960 & C & $\begin{array}{c}-55.02 \\
1961\end{array}$ & $\begin{array}{c}-58.15 \\
1962\end{array}$ & -59.33 & -59.80 & 2.16 & 4.72 & \\
\hline \multicolumn{13}{|c|}{ COLLIER COUNTY } \\
\hline 54 & B & 9 & 8 & 1951 & C & $\begin{array}{r}+13.1 \\
1958\end{array}$ & $\begin{array}{l}+8.05 \\
1962\end{array}$ & +11.34 & +12.96 & 3.54 & 2.18 & $M$ \\
\hline 131 & B & 54 & 22 & 1952 & C & $\begin{array}{r}+26.2 \\
1958\end{array}$ & $\begin{array}{c}+20.90 \\
1962\end{array}$ & +22.24 & +23.10 & 3.90 & 3.67 & $M$ \\
\hline 164 & B & 51 & 20 & 1958 & C & $\begin{array}{l}+5.5 \\
1959\end{array}$ & $\begin{array}{l}-0.85 \\
1962\end{array}$ & +2.60 & +0.73 & 3.95 & 3.36 & $\begin{array}{l}\text { M; Naples well } \\
\text { field }\end{array}$ \\
\hline 271 & B & 38 & -- & 1959 & $\mathrm{C}$ & $\begin{array}{l}-2.18 \\
1962\end{array}$ & $\begin{array}{l}-4.9 \\
1960\end{array}$ & -1.97 & -3.02 & 3.58 & 3.75 & B \\
\hline 296 & B & 45 & $\cdots$ & 1959 & C & $\begin{array}{l}-7.2 \\
1962\end{array}$ & $\begin{array}{l}-7.65 \\
1962\end{array}$ & -6.4 & -5.9 & 5.7 & 5.2 & \\
\hline \multicolumn{13}{|c|}{ COLUMBLA COUNTY } \\
\hline $9(010-238-1)$ & $\mathbf{F}$ & 836R & 680 & 1942 & C & $\begin{array}{c}-79.60 \\
1948\end{array}$ & $\begin{array}{c}-97.02 \\
1957\end{array}$ & -92.93 & -90.30 & 1.84 & 8.51 & \\
\hline \multicolumn{13}{|c|}{ DADE COUNTY } \\
\hline F45 & B & 85 & -- & 1939 & C & $\begin{array}{l}+3.9 \\
1960\end{array}$ & $\begin{array}{l}+1.6 \\
1960\end{array}$ & -2.32 & +2.30 & 3.15 & 3.05 & M; R, 1959 \\
\hline F179 & B & 77 & $\cdots$ & 1940 & C & $\begin{array}{l}+6.0 \\
1958\end{array}$ & $\begin{array}{l}+0.9 \\
1945\end{array}$ & +1.97 & +2.28 & 3.05 & 2.48 & $M$ \\
\hline F240 & B & 60 & -- & 1939 & C & $\cdots$ & $\cdots$ & $\cdots$ & +2.42 & 2.87 & 3.57 & $M ; R, 1961$ \\
\hline F319 & B & 17 & 13 & 1940 & C & $\begin{array}{l}+5.4 \\
1958\end{array}$ & $\begin{array}{l}+0.5 \\
1945\end{array}$ & +2.73 & +2.77 & 1.89 & 1.81 & $\mathbf{M}$ \\
\hline
\end{tabular}




\begin{tabular}{|c|c|c|c|c|c|c|c|c|c|c|c|}
\hline \multirow{4}{*}{ Well number } & \multirow{4}{*}{ 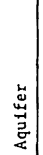 } & \multirow{4}{*}{ 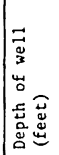 } & \multirow{4}{*}{ 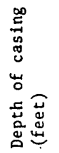 } & \multirow{4}{*}{ 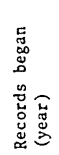 } & \multirow{4}{*}{ 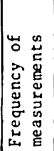 } & \multicolumn{5}{|c|}{$\begin{array}{c}\text { Water level above }(+) \text { or below }(-) \text { land surface } \\
\text { (feet) }\end{array}$} & \multirow{4}{*}{ Remarks } \\
\hline & & & & & & Prior to 1963 & \multirow{2}{*}{\multicolumn{2}{|c|}{$\begin{array}{l}\text { Highest water } \\
\text { level In May } \\
\text { or June }\end{array}$}} & \multirow{2}{*}{\multicolumn{2}{|c|}{$\begin{array}{l}\text { Annua 1 } \\
\text { Range }\end{array}$}} & \\
\hline & & & & & & May or June & & & & & \\
\hline & & & & & & $\left.\begin{array}{c|c}\text { High } \\
\text { (year) }\end{array}\right) \begin{array}{c}\text { Low } \\
\text { (year) }\end{array}$ & 1963 & 1964 & 1963 & 1964 & \\
\hline
\end{tabular}

\begin{tabular}{|c|c|c|c|c|c|c|c|c|c|c|c|c|c|}
\hline F358 & B & 54 & -- & 1940 & C & $\begin{array}{l}+6.70 \\
1954\end{array}$ & $\begin{array}{l}-0.04 \\
1962\end{array}$ & +1.27 & +2.30 & 6.10 & 4.31 & $\mathbf{M}$ & \\
\hline G3 & B & 20 & 11 & 1940 & C & $\begin{array}{l}+3.00 \\
1958\end{array}$ & $\begin{array}{l}-0.50 \\
1951\end{array}$ & +0.08 & -0.16 & 3.25 & 4.17 & M; & $\mathbf{P}$ \\
\hline $\mathrm{GlO}$ & B & 6 & 6 & 1940 & C & $\begin{array}{l}+6.00 \\
1958\end{array}$ & $\begin{array}{l}+0.50 \\
1945\end{array}$ & +2.76 & +3.00 & 2.50 & 3.31 & $M$ & \\
\hline G39 & B & 6 & 6 & 1939 & C & $\begin{array}{l}+7.20 \\
1958\end{array}$ & $\begin{array}{l}+0.94 \\
1962\end{array}$ & +2.33 & +2.73 & 3.19 & 3.07 & M; & $\mathrm{P}$ \\
\hline G72 & B & 5 & 4 & 1940 & C & $\begin{array}{l}+6.50 \\
1958\end{array}$ & $\begin{array}{l}+1.20 \\
1945\end{array}$ & +3.85 & +5.11 & 2.89 & 1.81 & $M$ & \\
\hline 6476 & B & 24 & 19 & 1947 & $\mathrm{C}$ & $\begin{array}{l}+5.50 \\
1958\end{array}$ & $\begin{array}{r}+0.40 \\
1950 \& 56\end{array}$ & +1.38 & +1.33 & 2.15 & 1.65 & M & \\
\hline G553 & B & 91 & 79 & 1947 & $\mathrm{C}$ & $\begin{array}{l}+8.60 \\
1958\end{array}$ & $\begin{array}{l}+0.97 \\
1962\end{array}$ & +2.42 & +2.53 & 5.44 & 2.71 & $\begin{array}{l}\text { M; } \\
\text { slo }\end{array}$ & $\begin{array}{l}\text { Casing } \\
\text { otted } 36^{\prime}-79^{\prime}\end{array}$ \\
\hline G580A & B & 22 & 4 & 1960 & C & $\begin{array}{l}-4.84 \\
1961\end{array}$ & $\begin{array}{l}+0.95 \\
1962\end{array}$ & +2.32 & +1.98 & 3.45 & 2.86 & M & \\
\hline G595 & B & 14 & 11 & 1949 & C & $\begin{array}{l}+8.50 \\
1958\end{array}$ & $\begin{array}{l}-1.88 \\
1962\end{array}$ & +2.09 & +1.46 & 6.34 & 3.30 & $M$ & $\mathrm{P}$ \\
\hline G596 & B & 13 & 11 & 1949 & C & $\begin{array}{l}+8.40 \\
1958\end{array}$ & $\begin{array}{l}+2.11 \\
1962\end{array}$ & +2.68 & +3.56 & 5.45 & 4.09 & $M$ & \\
\hline G613 & $B$ & 21 & 18 & 1950 & C & $\begin{array}{c}+5.50 \\
1954858\end{array}$ & $\begin{array}{l}-0.98 \\
1962\end{array}$ & +1.98 & +2.50 & 6.50 & 3.87 & $M$ & \\
\hline G614 & B & 20 & 18 & 1950 & $\mathrm{C}$ & $\begin{array}{l}+8.20 \\
1958\end{array}$ & $\begin{array}{l}+0.37 \\
1962\end{array}$ & +1.50 & +2.28 & 7.80 & 4.53 & $M$ & \\
\hline G618 & B & 20 & 11 & 1950 & C & $\begin{array}{l}+8.40 \\
1958\end{array}$ & $\begin{array}{l}+2.84 \\
1962\end{array}$ & +5.01 & +5.60 & 2.99 & 2.04 & $M$ & \\
\hline G619 & B & 12 & 6 & 1950 & C & $\begin{array}{l}+8.30 \\
1958\end{array}$ & $\begin{array}{l}+4.3 \\
1956\end{array}$ & +6.83 & +7.29 & 2.53 & 1.96 & $M$ & \\
\hline G620 & B & 16 & 6 & 1950 & C & $\begin{array}{l}+7.0 \\
1958\end{array}$ & $\begin{array}{l}+3.6 \\
1952\end{array}$ & +5.81 & +5.62 & 3.83 & 2.13 & $\mathbf{M}$ & \\
\hline G757A & B & 20 & 10 & 1957 & $\mathrm{C}$ & $\begin{array}{l}+9.30 \\
1958\end{array}$ & $\begin{array}{l}+1.50 \\
1962\end{array}$ & +1.90 & +2.80 & 6.96 & 4.35 & $\mathbf{M}$ & \\
\hline G789 & B & 20 & 10 & 1956 & C & $\begin{array}{l}+7.30 \\
1958\end{array}$ & $\begin{array}{l}+1.15 \\
1962\end{array}$ & +1.80 & +3.65 & 6.97 & 6.04 & $\mathbf{M}$ & \\
\hline G799 & B & 20 & 10 & 1956 & $\mathrm{C}$ & $\begin{array}{l}+7.80 \\
1958\end{array}$ & $\begin{array}{l}+1.65 \\
1962\end{array}$ & +2.82 & +2.80 & 3.62 & 3.53 & $M$ & $\mathrm{P}$ \\
\hline G850 & B & 20 & 10 & 1959 & C & $\begin{array}{l}+2.30 \\
1960\end{array}$ & $\begin{array}{l}+1.20 \\
1959\end{array}$ & +2.40 & $\cdots$ & 3.61 & -- & $\mathbf{M}$ & \\
\hline G851 & B & 18 & 11 & 1959 & C & $\begin{array}{l}+2.90 \\
1960\end{array}$ & $\begin{array}{l}+1.80 \\
1959\end{array}$ & +3.63 & +4.15 & 1.95 & 3.00 & $M$ & \\
\hline G852 & B & 20 & 10 & 1959 & C & $\begin{array}{l}+2.40 \\
1960\end{array}$ & $\begin{array}{l}+0.40 \\
1959\end{array}$ & +2.20 & +2.87 & 3.75 & 4.30 & $\mathbf{M}$ & \\
\hline G855 & B & 20 & 10 & 1958 & C & 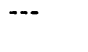 & $\begin{array}{l}-9.10 \\
1962\end{array}$ & -8.10 & -7.70 & 4.70 & 3.85 & B & \\
\hline G857 & B & 19 & 135 & 1959 & C & $\begin{array}{l}+3.70 \\
1960\end{array}$ & $\begin{array}{l}+1.30 \\
1962\end{array}$ & +2.40 & +2.75 & 3.88 & 3.10 & $\mathbf{M}$ & \\
\hline G858 & B & 20 & 11 & 1959 & C & $\begin{array}{l}+6.30 \\
1960\end{array}$ & $\begin{array}{l}+1.82 \\
1962\end{array}$ & +2.65 & +2.70 & 6.25 & 2.95 & $\mathbf{M}$ & \\
\hline
\end{tabular}




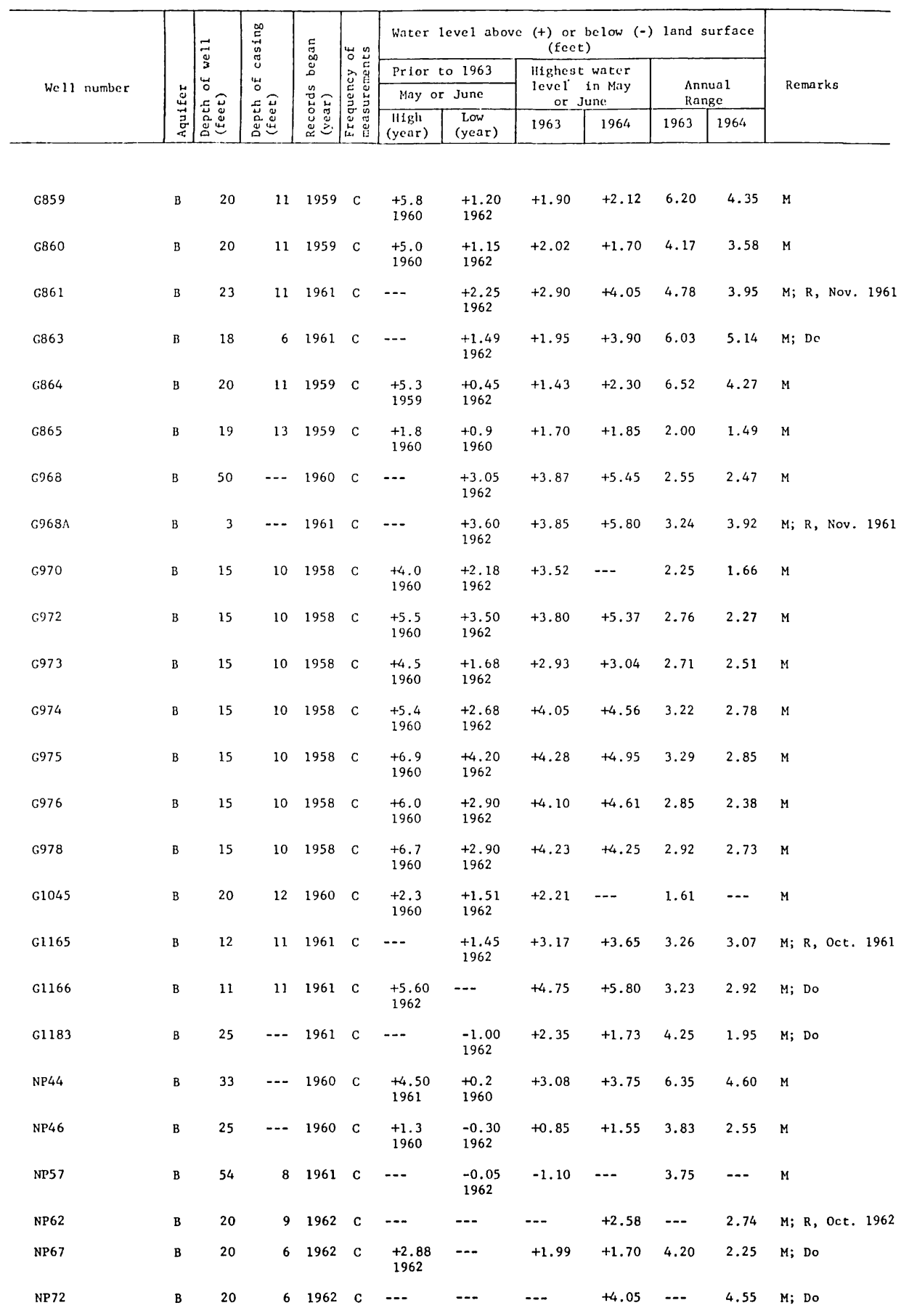




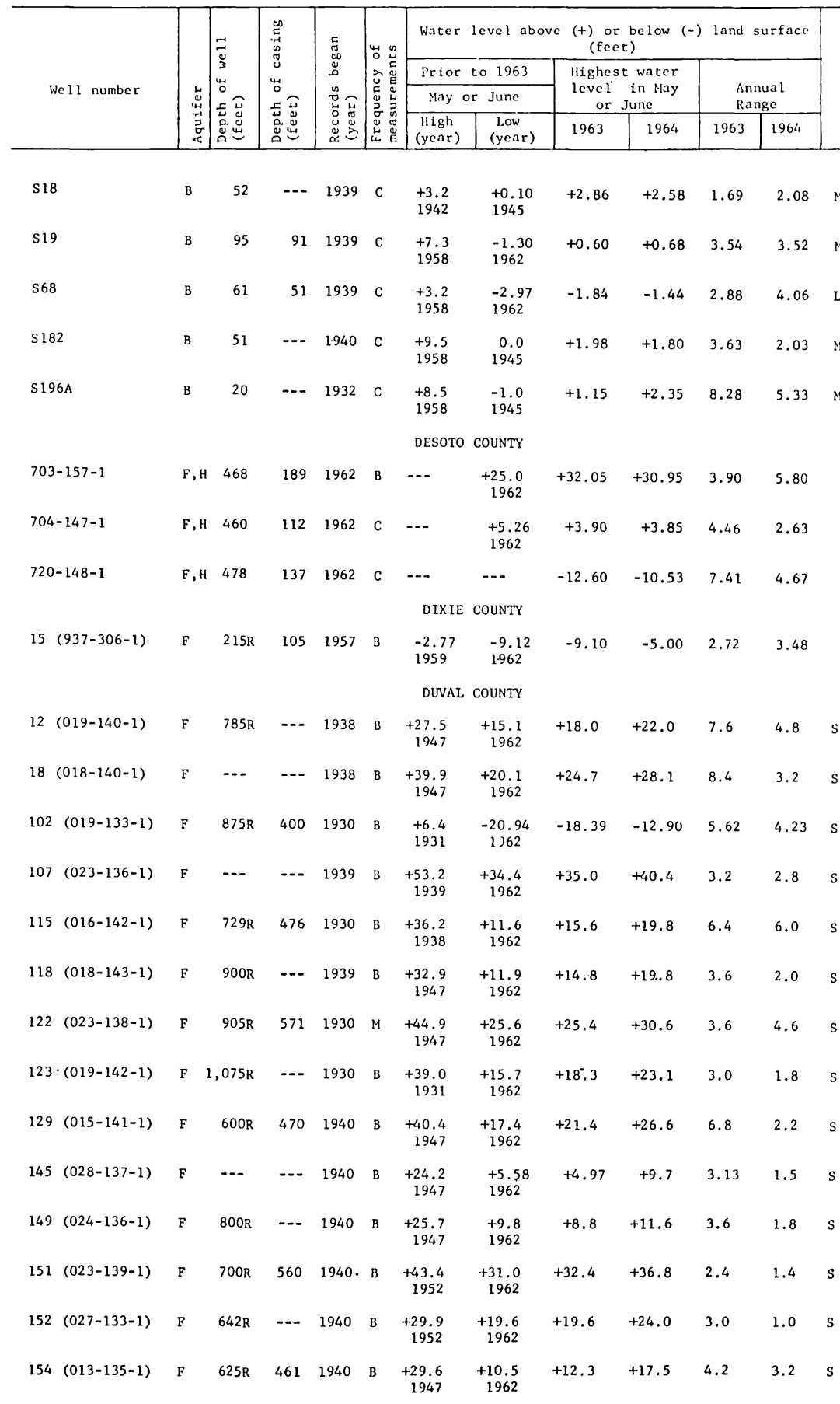




\begin{tabular}{|c|c|c|c|c|c|c|c|c|c|c|c|c|}
\hline \multirow{4}{*}{ We 11 number } & \multirow{4}{*}{. } & \multirow{4}{*}{ 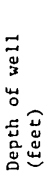 } & \multirow{4}{*}{ 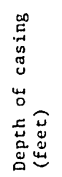 } & \multirow{4}{*}{ 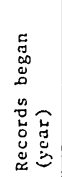 } & \multirow{4}{*}{ 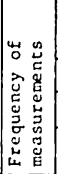 } & \multicolumn{6}{|c|}{$\begin{array}{c}\text { Water level above }(+) \text { or below }(-) \text { land surface } \\
(\text { feet })\end{array}$} & \multirow{4}{*}{ Remarks } \\
\hline & & & & & & \multirow{2}{*}{\multicolumn{2}{|c|}{$\frac{\text { Prior to } 1963}{\text { May or June }}$}} & \multirow{2}{*}{\multicolumn{2}{|c|}{$\begin{array}{c}\text { Highest water } \\
\text { level in May } \\
\text { or June }\end{array}$}} & \multirow{2}{*}{\multicolumn{2}{|c|}{$\begin{array}{l}\text { Annual } \\
\text { Range }\end{array}$}} & \\
\hline & & & & & & & & & & & & \\
\hline & & & & & & $\begin{array}{l}\text { lligh } \\
\text { (year) }\end{array}$ & $\begin{array}{l}\text { Low } \\
\text { (year) }\end{array}$ & 1963 & 1964 & 1963 & 1964 & \\
\hline
\end{tabular}

\begin{tabular}{|c|c|c|c|c|c|c|c|c|c|c|c|c|c|}
\hline 160 & $(018-123-1)$ & F & $585 R$ & 357 & 1934 & B & $\begin{array}{r}+41.7 \\
1934\end{array}$ & $\begin{array}{r}+20.2 \\
1962\end{array}$ & +23.9 & +28.9 & 6.0 & 4.2 & $\mathrm{~S}, \mathrm{~T}$ \\
\hline 164 & $(025-125-1)$ & $\mathrm{F}$ & $840 R$ & 450 & 1930 & B & $\begin{array}{r}+43.8 \\
1931\end{array}$ & $\begin{array}{r}+25.8 \\
1962\end{array}$ & +26.9 & +30.5 & 3.6 & 0.6 & $\mathrm{~S}, \mathrm{~T}$ \\
\hline 206 & $(015-145-1)$ & $\mathrm{F}$ & $1,920 \mathrm{R} \quad 1$ & 1,000 & 1941 & C & $\begin{array}{l}-2.06 \\
1948\end{array}$ & $\begin{array}{c}-16.75 \\
1962\end{array}$ & -- & -12.95 & 2.30 & 5.03 & $\mathrm{~S}$ \\
\hline 262 & $(026-135-1)$ & $\mathrm{F}$ & $1,393 \mathrm{R}$ & 584 & 1951 & B & $\begin{array}{r}+37.0 \\
1951\end{array}$ & $\begin{array}{r}+23.5 \\
1962\end{array}$ & +23.4 & +28.0 & 2.6 & 3.6 & $S, T$ \\
\hline 263 & $(026-135-2)$ & F & $1,025 \mathrm{R}$ & 850 & 1951 & B & $\begin{array}{r}+35.5 \\
1952\end{array}$ & $\begin{array}{r}+24.2 \\
1962\end{array}$ & +24.0 & +28.6 & 3.0 & 1.8 & $S, T$ \\
\hline 264 & $(026-135-3)$ & F & $700 \mathrm{R}$ & 450 & 1951 & B & $\begin{array}{r}+35.3 \\
1952\end{array}$ & $\begin{array}{r}+23.2 \\
1962\end{array}$ & +23.2 & +28.0 & 2.6 & 1.6 & S, T \\
\hline 265 & $(025-136 \cdot 1)$ & $\mathrm{F}$ & $556 \mathrm{R}$ & -- & 1951 & B & $\begin{array}{r}+39.4 \\
1952\end{array}$ & $\begin{array}{r}+22.3 \\
1962\end{array}$ & +19.4 & +33.6 & 9.0 & 4.2 & $\mathrm{~S}, \mathrm{~T}$ \\
\hline \multicolumn{14}{|c|}{ ESCAMBIA COUNTY } \\
\hline 391 & $(023-716-2)$ & G & 244 & -- & 1940 & $M$ & $\begin{array}{l}-4.59 \\
1940\end{array}$ & $\begin{array}{c}-12.00 \\
1955\end{array}$ & -12.16 & -10.66 & 5.16 & 10.68 & \\
\hline 45( & $(036-719-1)$ & G & 152 & 129 & 1940 & C & $\begin{array}{c}-69.30 \\
1941\end{array}$ & $\begin{array}{c}-111.82 \\
1956\end{array}$ & -100.60 & -103.98 & 3.03 & 1.57 & $\mathrm{P}$ \\
\hline 46 & $(031-716-1)$ & G & 239 & 239 & 1939 & $w$ & $\begin{array}{c}-58.09 \\
1948\end{array}$ & $\begin{array}{l}-82.12 \\
1956\end{array}$ & -73.27 & -67.22 & 2.43 & 6.79 & \\
\hline 62 & $(024-715-1)$ & G & $142 \mathrm{R}$ & 142 & 1940 & $\mathrm{C}$ & $\begin{array}{l}-6.50 \\
1949\end{array}$ & $\begin{array}{c}-23.84 \\
1955\end{array}$ & -12.96 & -11.00 & 4.13 & 5.41 & \\
\hline $62 \mathrm{~A}$ & $(024-715-2)$ & G & 18 & 18 & 1940 & W & $\begin{array}{c}-10.22 \\
1944\end{array}$ & $\begin{array}{c}-13.05 \\
1962\end{array}$ & -11.12 & -8.66 & 0.75 & 3.26 & \\
\hline 73( & $(035-715-3)$ & $G$ & 306 & 198 & 1951 & $\mathrm{C}$ & $\begin{array}{c}-39.03 \\
1953\end{array}$ & $\begin{array}{c}-56.66 \\
1958\end{array}$ & -52.65 & -53.90 & 5.26 & 4.07 & $P$ \\
\hline 74 & $(036-716-1)$ & G & 352 & $350 *$ & 1951 & $\mathrm{C}$ & $\begin{array}{l}-77.37 \\
1952\end{array}$ & $\begin{array}{c}-89.52 \\
1959\end{array}$ & -87.97 & -86.53 & 1.59 & $\begin{array}{l}2.74 \\
\text { and }\end{array}$ & $\begin{array}{l}\mathrm{P} * \text { Screened from } \\
260 \text { to } 270 \text { feet } \\
340 \text { to } 350 \text { feet }\end{array}$ \\
\hline 83 & $(035-714-3)$ & G & 301 & -- & 1954 & C & $\begin{array}{c}-36.10 \\
1955\end{array}$ & $\begin{array}{l}-42.45 \\
1962\end{array}$ & -37.97 & -40.38 & 7.09 & 4.19 & $\mathrm{P}$ \\
\hline $026-$ & $-713-5$ & G & 149 & $144 *$ & 1959 & W & $\begin{array}{c}-58.15 \\
1960\end{array}$ & $\begin{array}{c}-60.35 \\
1962\end{array}$ & -63.57 & -59.92 & 2.20 & 4.82 & $\begin{array}{l}\text { *Screened from } \\
144 \text { to } 149 \text { feet }\end{array}$ \\
\hline $026-$ & $-713-6$ & G & 65 & $60 *$ & 1959 & W & $\begin{array}{c}-51.78 \\
1960\end{array}$ & $\begin{array}{c}-52.56 \\
1962\end{array}$ & -56.81 & -53.75 & 2.99 & 5.72 & $\begin{array}{l}\text { * Screened from } \\
60 \text { to } 65 \text { feet }\end{array}$ \\
\hline 032 & $-724-1$ & $G$ & 170 & $165 *$ & 1959 & $M$ & $\begin{array}{c}-91.18 \\
1960\end{array}$ & $\begin{array}{c}-91.93 \\
1962\end{array}$ & -93.04 & -92.20 & 1.35 & 1.73 & $\begin{array}{l}\text { *Screened from } \\
165 \text { to } 170 \text { feet }\end{array}$ \\
\hline $054-$ & $-726-1$ & G & 206 & $201 \star$ & 1959 & B & $\begin{array}{l}-82.95 \\
1962\end{array}$ & $\begin{array}{c}-89.48 \\
1959\end{array}$ & -87.90 & -90.06 & 2.98 & 3.00 & $\begin{array}{l}\text { *Screened from } \\
201 \text { to } 206 \text { feet }\end{array}$ \\
\hline 054 & $-726-2$ & G & 107 & $102 \star$ & 1959 & B & $\begin{array}{l}-65.21 \\
1962\end{array}$ & $\begin{array}{c}-74.92 \\
1959\end{array}$ & -72.50 & -76.15 & 3.79 & 4.78 & $\begin{array}{l}\text { ^Screened from } \\
102 \text { to } 107 \text { feet }\end{array}$ \\
\hline & & & & & & & FLAGLER & COUNTY & & & & & \\
\hline 14 & $(927-115-1)$ & $\mathrm{F}$ & 417 & -- & 1936 & B & $\begin{array}{l}-3.4 \\
1937\end{array}$ & $\begin{array}{l}-8.19 \\
1962\end{array}$ & -7.55 & -6.62 & 2.32 & 2.11 & \\
\hline
\end{tabular}




\begin{tabular}{|c|c|c|c|c|c|c|c|c|c|c|c|c|}
\hline \multirow{4}{*}{ We11 number } & \multirow{4}{*}{ 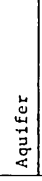 } & \multirow{4}{*}{ 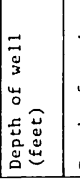 } & \multirow{4}{*}{ 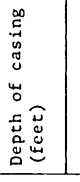 } & \multirow{4}{*}{ 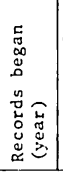 } & \multirow{4}{*}{ 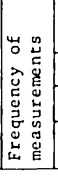 } & \multicolumn{6}{|c|}{$\begin{array}{c}\text { Water level above }(+) \text { or below }(-) \text { land surface } \\
(\text { feet })\end{array}$} & \multirow{4}{*}{ Remarks } \\
\hline & & & & & & \multirow{2}{*}{\multicolumn{2}{|c|}{$\begin{array}{l}\text { Prior to } 1963 \\
\text { May or June }\end{array}$}} & \multirow{2}{*}{\multicolumn{2}{|c|}{$\begin{array}{c}\text { Highest water } \\
\text { level in May } \\
\text { or June }\end{array}$}} & \multirow{2}{*}{\multicolumn{2}{|c|}{$\begin{array}{l}\text { Annual } \\
\text { Range }\end{array}$}} & \\
\hline & & & & & & & & & & & & \\
\hline & & & & & & $\begin{array}{l}\text { High } \\
\text { (year) }\end{array}$ & $\begin{array}{l}\text { Low } \\
\text { (year) }\end{array}$ & 1963 & 1964 & 1963 & 1964 & \\
\hline $44(928-122-1)$ & $\mathbf{F}$ & 159 & --- & 1956 & B & $\begin{array}{l}-7.67 \\
1959 \\
\text { FRANKLII }\end{array}$ & $\begin{array}{c}-13.42 \\
1962 \\
\text { IN COUNTY }\end{array}$ & -11.38 & -9.31 & 7.13 & 2.72 & $P$ \\
\hline $10(950-439-1)$ & $\mathrm{F}$ & $380 \mathrm{R}$ & -- & 1958 & B & $\begin{array}{l}-1.80 \\
1961\end{array}$ & $\begin{array}{l}-4.45 \\
1962\end{array}$ & -2.33 & -0.35 & 2.43 & 3.27 & \\
\hline $31(943-458-1)$ & $\mathbf{F}$ & $\cdots$ & -- & 1949 & B & $\begin{array}{l}+3.95 \\
1950\end{array}$ & $\begin{array}{l}+0.40 \\
1952\end{array}$ & +1.75 & +3.4 & 0.65 & 1.20 & \\
\hline $947-446-1$ & $\mathbf{F}$ & $98 \mathrm{R}$ & --- & 1961 & B & $\cdots$ & $\begin{array}{c}-11.26 \\
1962\end{array}$ & -11.35 & -9.67 & 1.03 & 1.22 & \\
\hline $957-443-1$ & $\mathbf{F}$ & -- & $\cdots$ & 1961 & $\mathrm{~B}$ & -- & $\begin{array}{l}+2.97 \\
1962\end{array}$ & +3.87 & +4.87 & 0.90 & 1.00 & \\
\hline \multicolumn{13}{|c|}{ GADSDEN COUNTY } \\
\hline $035-435-1$ & $F$ & $406 \mathrm{R}$ & $\cdots$ & 1961 & B & -- & $\begin{array}{l}-90.96 \\
1962\end{array}$ & -91.40 & -90.76 & 9.86 & 0.12 & \\
\hline $039-425-1$ & $\mathbf{F}$ & $525 R$ & 381 & 1961 & B & $\cdots$ & $\begin{array}{l}-143.96 \\
1962\end{array}$ & --- & -- & 1.71 & $\cdots$ & \\
\hline \multicolumn{13}{|c|}{ GULF COUNTY } \\
\hline $30(948-518-1)$ & $F$ & 522 & 475 & 1946 & $\mathrm{C}$ & $\begin{array}{l}-7.11 \\
1956\end{array}$ & $\begin{array}{c}-27.22 \\
1950\end{array}$ & -9.40 & -7.82 & 3.31 & 2.15 & $\begin{array}{l}\text { P, prior to } \\
1954\end{array}$ \\
\hline $33(939-521-1)$ & $\mathbf{F}$ & 595 & 487 & 1961 & B & $\cdots$ & $\begin{array}{l}+1.29 \\
1962\end{array}$ & +0.96 & +0.99 & 0.62 & 1.15 & \\
\hline $34(006-511-1)$ & $\mathrm{F}$ & $578 R$ & 248 & 1961 & B & $\cdots$ & $\begin{array}{r}-10.0 \\
1962\end{array}$ & +9.5 & $\cdots$ & 4.5 & $\cdots$ & P; D, 1964 \\
\hline \multicolumn{13}{|c|}{ HAMILTON COUNTY } \\
\hline $036-305-1$ & $\mathbf{F}$ & $273 R$ & 60 & 1961 & $B$ & $\cdots$ & $\begin{array}{c}-101.78 \\
1962\end{array}$ & -107.05 & -84.73 & 11.03 & 23.83 & \\
\hline \multicolumn{13}{|c|}{ HARDEE COUNTY } \\
\hline $731-145-1$ & $\mathbf{F}$ & 450 & 39 & 1962 & $\mathrm{C}$ & $\cdots$ & $\begin{array}{c}-33.60 \\
1962\end{array}$ & -33.24 & -29.56 & 11.08 & 16.00 & \\
\hline \multicolumn{13}{|c|}{ HENDRY COUNTY } \\
\hline 3 & $\mathrm{~s}$ & 10 & 8 & 1941 & $\mathrm{C}$ & $\begin{array}{l}+0.3 \\
1958\end{array}$ & $\begin{array}{l}-5.76 \\
1962\end{array}$ & -1.15 & -2.62 & 3.95 & 4.33 & \\
\hline 5 & $\mathrm{~s}$ & 13 & 8 & 1941 & C & $\begin{array}{l}-0.81 \\
1959\end{array}$ & $\begin{array}{l}-6.3 \\
1956\end{array}$ & -3.42 & -3.29 & 1.73 & 2.7 & \\
\hline \multicolumn{13}{|c|}{ HERNANDO COUNTY } \\
\hline $838-215-1$ & F & $140 \mathrm{R}$ & -- & 1961 & B & $\cdots$ & $\begin{array}{c}-20.46 \\
1962\end{array}$ & -19.35 & -16.30 & 1.55 & 3.72 & \\
\hline \multicolumn{13}{|c|}{ HIGHLANDS COUNTY } \\
\hline 9 & $\mathrm{~s}$ & 26 & 22 & 1948 & $\mathrm{C}$ & $\begin{array}{r}+130.4 \\
1953\end{array}$ & $\begin{array}{r}+126.0 \\
1949\end{array}$ & +128.32 & +129.34 & 2.84 & 3.09 & $M$ \\
\hline 10 & $\mathrm{~s}$ & 45 & 41 & 1948 & $\mathrm{C}$ & $\begin{array}{r}+90.7 \\
1958\end{array}$ & $\begin{array}{r}+83.9 \\
1956\end{array}$ & +88.45 & +87.06 & 2.5 & 1.65 & $M$ \\
\hline $11 \mathrm{~A}$ & $\mathrm{~S}$ & 10 & 8 & 1956 & $\mathrm{C}$ & $\begin{array}{r}+48.3 \\
1957\end{array}$ & $\begin{array}{c}+43.71 \\
1962\end{array}$ & +46.37 & +47.99 & 3.12 & 2.66 & M \\
\hline 13 & $\mathrm{~s}$ & 20 & 16 & 1948 & $\mathrm{C}$ & $\begin{array}{r}+28.9 \\
1957\end{array}$ & $\begin{array}{c}+20.57 \\
1962\end{array}$ & +24.16 & +23.72 & 2.77 & 4.84 & $M$ \\
\hline 14 & $\mathrm{~s}$ & 35 & 29 & 1948 & C & $\begin{array}{c}+122.19 \\
1960\end{array}$ & $\begin{array}{r}+114.7 \\
1951\end{array}$ & +120.25 & +118.75 & 2.73 & 2.28 & $M$ \\
\hline
\end{tabular}




\begin{tabular}{|c|c|c|c|c|c|c|c|c|c|c|c|c|}
\hline \multirow{4}{*}{ We 11 number } & \multirow{4}{*}{ 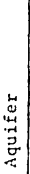 } & \multirow{4}{*}{ 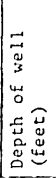 } & \multirow{4}{*}{ 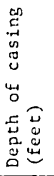 } & \multirow{4}{*}{ 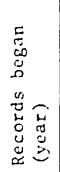 } & \multirow{4}{*}{ 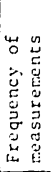 } & \multicolumn{6}{|c|}{$\begin{array}{c}\text { Water level above: }(+) \text { or below }(-) \text { land surface } \\
\text { (feet) }\end{array}$} & \multirow{4}{*}{ Remarks } \\
\hline & & & & & & \multicolumn{2}{|c|}{ Prior to 1963} & \multirow{2}{*}{\multicolumn{2}{|c|}{$\begin{array}{c}\text { llighest water } \\
\text { level in May } \\
\text { or June }\end{array}$}} & \multirow{2}{*}{\multicolumn{2}{|c|}{$\begin{array}{l}\text { Annual } \\
\text { Range }\end{array}$}} & \\
\hline & & & & & & \multicolumn{2}{|c|}{ May or June } & & & & & \\
\hline & & & & & & $\begin{array}{c}\text { llgh } \\
\text { (year) }\end{array}$ & $\begin{array}{l}\text { Low } \\
\text { (year) }\end{array}$ & 1963 & 1964 & 1963 & 1964 & \\
\hline
\end{tabular}

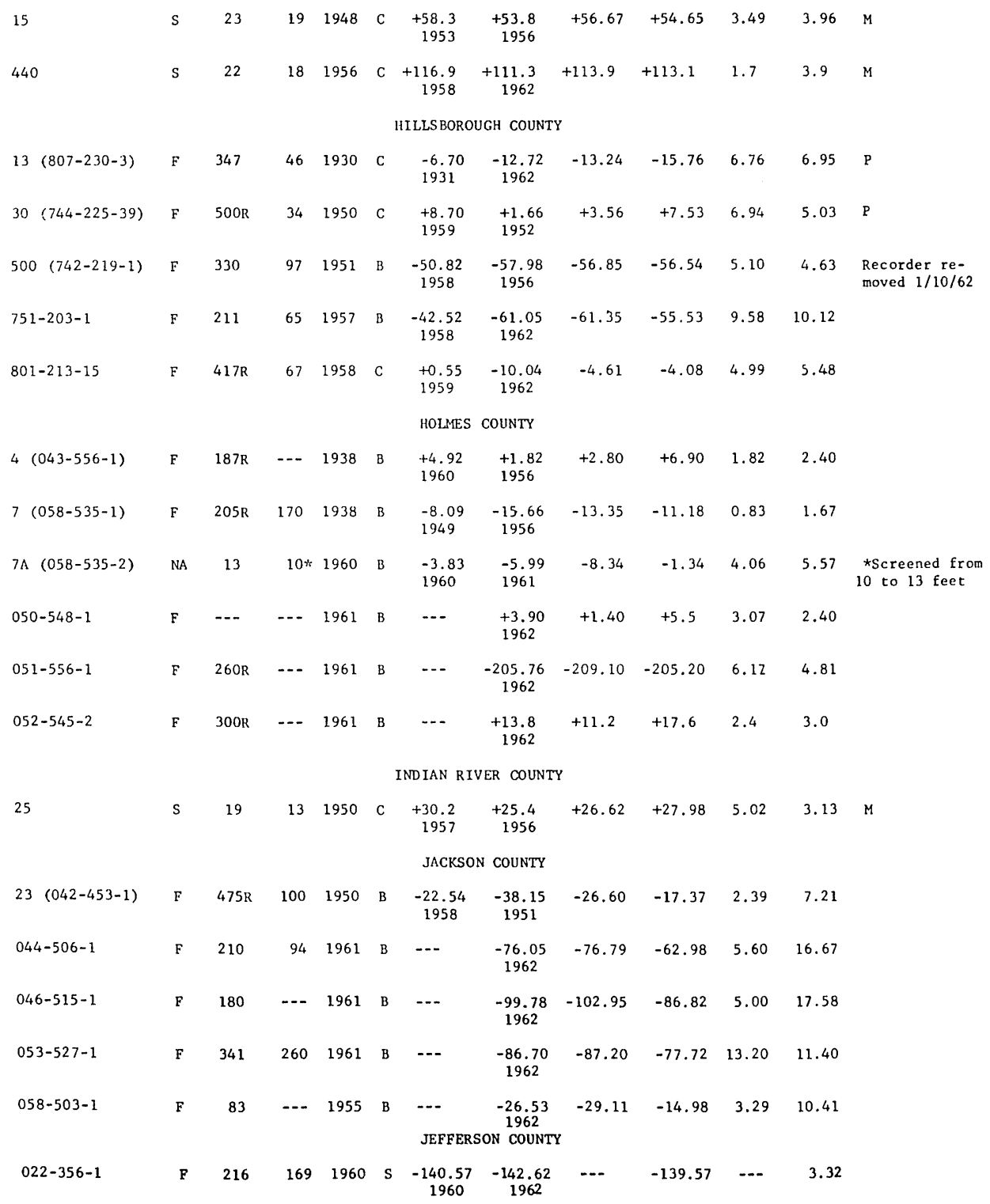




\begin{tabular}{|c|c|c|c|c|c|c|c|c|c|c|}
\hline \multirow{4}{*}{ We 11 number } & \multirow{4}{*}{ 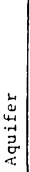 } & \multirow{4}{*}{ 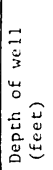 } & \multirow{4}{*}{ 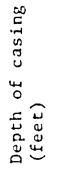 } & \multirow{4}{*}{ 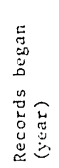 } & \multirow{4}{*}{ 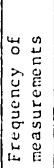 } & \multicolumn{4}{|c|}{$\begin{array}{l}\text { Water level above }(+) \text { or below }(-) \text { land surface } \\
\text { (feet) }\end{array}$} & \multirow{4}{*}{ Remarks } \\
\hline & & & & & & Prior to 1963 & \multirow{2}{*}{$\begin{array}{l}\text { Highest water } \\
\text { level in May } \\
\text { or Jung. }\end{array}$} & \multirow{2}{*}{\multicolumn{2}{|c|}{$\begin{array}{l}\text { Annual } \\
\text { Range }\end{array}$}} & \\
\hline & & & & & & May or June & & & & \\
\hline & & & & & & $\begin{array}{l}\text { Low } \\
(y e a r)\end{array}$ & 1963 & 1963 & 1964 & \\
\hline
\end{tabular}

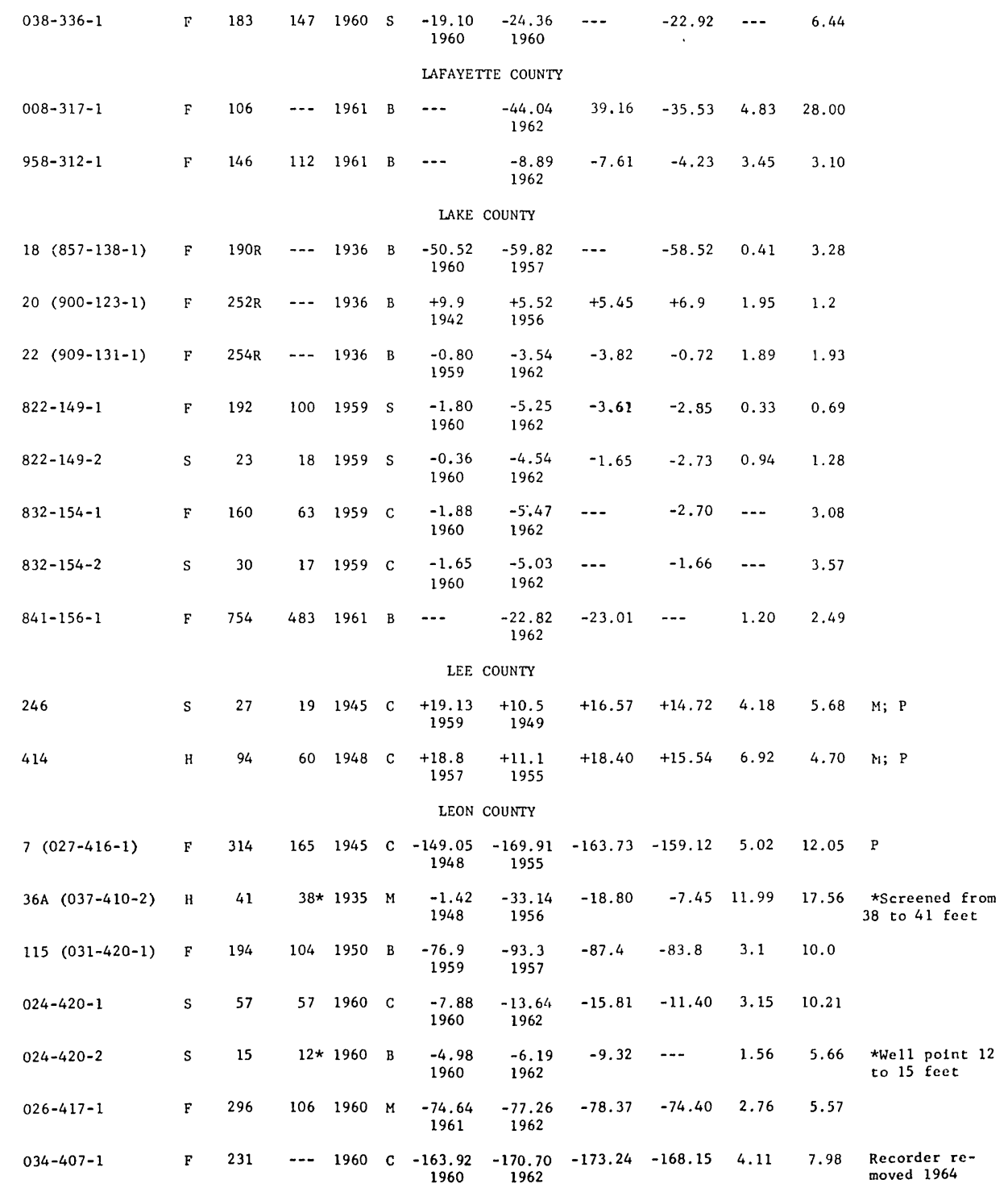




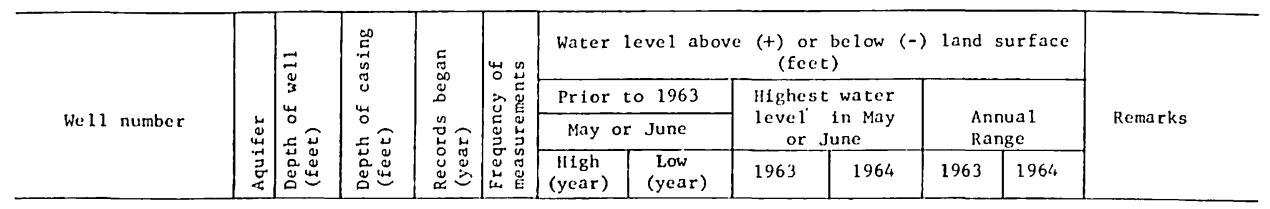

LEVY COUNTY

\begin{tabular}{|c|c|c|c|c|c|c|c|c|c|c|c|c|}
\hline $902-241-1$ & $\mathrm{~F}$ & 58 & $\cdots$ & 1961 & B & $\cdots$ & $\begin{array}{l}-8.34 \\
1962\end{array}$ & -7.99 & -5.80 & 1.31 & 6.89 & \\
\hline $919-245-1$ & $\mathrm{~F}$ & $96 \mathrm{R}$ & -- & 1961 & B & -- & $\begin{array}{l}-0.55 \\
1962\end{array}$ & -0.65 & -0.68 & 0.06 & 0.02 & \\
\hline \multicolumn{13}{|c|}{ LIBERTY COUNTY } \\
\hline $14(001-459-1)$ & $\mathbf{F}$ & --- & -- & 1955 & B & $\begin{array}{l}-4.68 \\
1961\end{array}$ & $\begin{array}{l}-7.12 \\
1961\end{array}$ & -6.62 & -3.60 & 3.17 & 2.23 & \\
\hline $15(022-841-1)$ & $\mathbf{F}$ & 395 & 242 & 1960 & C & $\begin{array}{c}-23.05 \\
1961\end{array}$ & $\begin{array}{c}-25.96 \\
1962\end{array}$ & -24.91 & -21.81 & 3.38 & 3.09 & D, 1964 \\
\hline $010-440-1$ & $\mathbf{F}$ & $118 \mathrm{R}$ & 89 & 1961 & B & $\begin{array}{l}+7.2 \\
1962\end{array}$ & $\begin{array}{r}+6.8 \\
1961\end{array}$ & +8.60 & +13.0 & 1.8 & 4.0 & D, 1964 \\
\hline $023-447-1$ & $F$ & $160 R$ & -- & 1961 & B & $\begin{array}{l}+2.90 \\
1962\end{array}$ & $\begin{array}{l}+2.8 \\
1961\end{array}$ & +3.40 & +4.80 & 1.25 & 1.70 & \\
\hline $028-456-1$ & $\mathrm{~F}$ & 360 & --- & 1961 & B & $\begin{array}{c}-84.73 \\
1961\end{array}$ & $\begin{array}{c}-85.64 \\
1962\end{array}$ & -85.50 & -83.82 & 0.40 & 1.88 & \\
\hline \multicolumn{13}{|c|}{ MADISON COUNTY } \\
\hline $17(028-325-1)$ & $\mathbf{F}$ & 320 & 300 & 1953 & B & $\begin{array}{c}-20.16 \\
1959\end{array}$ & $\begin{array}{c}-38.12 \\
1955\end{array}$ & -34.04 & --- & 3.93 & 20.43 & \\
\hline $18(028-325-2)$ & $\mathbf{F}$ & 322 & 307 & 1952 & C & $\begin{array}{c}-18.18 \\
1960\end{array}$ & $\begin{array}{c}-34.87 \\
1955\end{array}$ & -28.59 & -17.16 & 5.34 & 19.10 & $\mathbf{P}$ \\
\hline \multicolumn{13}{|c|}{ MANATEE COUNTY } \\
\hline $92(726-218-1)$ & $\mathbf{F}$ & 600 & 154 & 1941 & B & $\begin{array}{c}-37.10 \\
1947\end{array}$ & $\begin{array}{c}-52.65 \\
1962\end{array}$ & -49.35 & -46.76 & 9.52 & 2.41 & $\mathrm{~S}$ \\
\hline & & & & & & MARION & COUNTY & & & & & \\
\hline $5(911-159-1)$ & $\mathbf{F}$ & $135 R$ & 135 & 1933 & C & $\begin{array}{c}+13.62 \\
1960\end{array}$ & $\begin{array}{l}+3.35 \\
1957\end{array}$ & +5.99 & +9.24 & 1.16 & 8.57 & \\
\hline $47(902-156-1)$ & $\mathbf{F}$ & 179 & -- & 1936 & $\mathrm{~B}$ & $\begin{array}{c}-13.84 \\
1960\end{array}$ & $\begin{array}{c}-24.26 \\
1956\end{array}$ & -22.53 & -19.55 & 0.97 & 6.44 & \\
\hline $48(859-150-1)$ & $\mathbf{F}$ & 152 & $\cdots$ & 1936 & B & $\begin{array}{l}-0.82 \\
1961\end{array}$ & $\begin{array}{c}-10.23 \\
1956\end{array}$ & -7.35 & -5.75 & 0.68 & 4.42 & $\begin{aligned} \text { Well flowed } & \text { foril } 1960- \\
\text { April } & 1961\end{aligned}$ \\
\hline $49(910-138-1)$ & $\mathbf{F}$ & 175 & --- & 1936 & B & $\begin{array}{r}-25.0 \\
1942\end{array}$ & $\begin{array}{c}-31.19 \\
1957\end{array}$ & -30.53 & -28.37 & 1.10 & 3.38 & ' \\
\hline $51(911-210-1)$ & $\mathbf{F}$ & 106 & $\cdots$ & 1935 & B & $\begin{array}{c}-26.04 \\
1960\end{array}$ & $\begin{array}{c}-34.39 \\
1956\end{array}$ & -32.89 & -29.11 & 1.58 & 11.11 & \\
\hline \multicolumn{13}{|c|}{ MARTIN COUNTY } \\
\hline 140 & $\mathrm{~S}$ & 31 & 20 & 1950 & C & $\begin{array}{r}+20.2 \\
1957\end{array}$ & $\begin{array}{c}+15.77 \\
1961\end{array}$ & +18.89 & +18.40 & 4.19 & 3.27 & M \\
\hline 147 & $\mathbf{S}$ & 74 & 73 & 1952 & C & $\begin{array}{l}+9.8 \\
1958\end{array}$ & $\begin{array}{l}+2.12 \\
1962\end{array}$ & +2.28 & +4.61 & 6.09 & 6.45 & $\mathrm{M} ; \mathrm{P}$ \\
\hline 928 & $\mathbf{S}$ & 11 & 10 & 1957 & C & $\begin{array}{r}+32.4 \\
1957\end{array}$ & $\begin{array}{c}+28.40 \\
1962\end{array}$ & +28.05 & +30.90 & 2.40 & 2.80 & M \\
\hline 933 & $\mathrm{~S}$ & 15 & 14 & 1957 & C & $\begin{array}{r}+23.4 \\
1960\end{array}$ & $\begin{array}{c}+21.05 \\
1962\end{array}$ & +20.40 & +21.60 & 4.20 & 3.60 & $M$ \\
\hline
\end{tabular}




\begin{tabular}{|c|c|c|c|c|c|c|c|c|c|c|c|c|}
\hline \multirow{4}{*}{ We 11 number } & \multirow{4}{*}{ 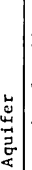 } & \multirow{4}{*}{ 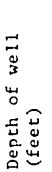 } & \multirow{4}{*}{ 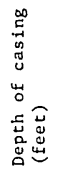 } & \multirow{4}{*}{ 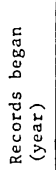 } & \multirow{4}{*}{ 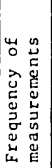 } & \multicolumn{6}{|c|}{$\begin{array}{c}\text { Water level above }(+) \text { or below }(-) \text { land surface } \\
\text { (feet) }\end{array}$} & \multirow{4}{*}{ Remarks } \\
\hline & & & & & & \multicolumn{2}{|c|}{ Prior to 1963} & \multirow{2}{*}{\multicolumn{2}{|c|}{$\begin{array}{l}\text { Highest water } \\
\text { leve } 1^{-} \text {in May } \\
\text { or June }\end{array}$}} & \multirow{2}{*}{\multicolumn{2}{|c|}{$\begin{array}{l}\text { Annual } \\
\text { Range }\end{array}$}} & \\
\hline & & & & & & \multicolumn{2}{|c|}{ May or June } & & & & & \\
\hline & & & & & & $\begin{array}{l}\text { lligh } \\
\text { (year) }\end{array}$ & $\begin{array}{l}\text { Low } \\
\text { (year) }\end{array}$ & 1963 & 1964 & 1963 & 1964 & \\
\hline
\end{tabular}

NASSAU COUNTY

\begin{tabular}{|c|c|c|c|c|c|c|c|c|c|c|c|c|c|}
\hline 2 & $(035-127-2)$ & $\mathrm{F}$ & $580 \mathrm{R}$ & 350 & 1939 & B & $\begin{array}{r}+42.0 \\
1947\end{array}$ & $\begin{array}{r}+20.8 \\
1962\end{array}$ & +18.4 & +24.6 & 4.4 & 2.0 & $\mathrm{~s}$ \\
\hline 8 & $(032-126-1)$ & F & $680 \mathrm{R}$ & $\cdots$ & 1939 & B & $\begin{array}{r}+41.1 \\
1947\end{array}$ & $\begin{array}{r}+20.6 \\
1962\end{array}$ & +21.4 & +26.0 & 3.0 & 1.2 & $\mathrm{P}$ \\
\hline 12 & $(038-127-1)$ & $\mathbf{F}$ & $640 \mathrm{R}$ & -- & 1939 & B & $\begin{array}{r}+24.0 \\
1947\end{array}$ & $\begin{array}{c}-17.23 \\
1956\end{array}$ & -18.26 & -7.93 & 22.15 & 3.89 & $\mathrm{P}$ \\
\hline 27 & $(040-126-1)$ & $\mathbf{F}$ & 191 & -- & 1939 & B & $\begin{array}{r}+10.1 \\
1946\end{array}$ & $\begin{array}{c}-26.10 \\
1957\end{array}$ & -29.34 & -23.06 & 9.97 & 13.41 & $\mathrm{~S}$ \\
\hline 44 & $(037-136-1)$ & $\mathbf{F}$ & $1,000 \mathrm{R}$ & 450 & 1934 & B & $\begin{array}{r}+19.8 \\
1947\end{array}$ & $\begin{array}{l}+0.26 \\
1962\end{array}$ & -2.13 & --- & 3.05 & $-\cdots$ & $\mathrm{S}$ \\
\hline 50 & $(036-142-1)$ & $\mathbf{F}$ & $569 \mathrm{R}$ & $\cdots$ & 1940 & B & $\begin{array}{r}+40.5 \\
1940\end{array}$ & $\begin{array}{r}+22.1 \\
1962\end{array}$ & +19.8 & +21.0 & 4.0 & 0.8 & $\mathrm{~S}$ \\
\hline 51 & $(033-150-1)$ & F & $580 \mathrm{R}$ & $\cdots$ & 1940 & B & $\begin{array}{l}+42.0 \\
1947 \& 48\end{array}$ & $\begin{array}{r}+26.2 \\
1962\end{array}$ & +25.2 & +29.8 & 1.8 & 3.3 & $\mathrm{~S}$ \\
\hline 55 & $(037-130-1)$ & F & 540R & 504 & 1940 & B & $\begin{array}{r}+33.1 \\
1947\end{array}$ & $\begin{array}{l}+8.5 \\
1957\end{array}$ & +4.9 & +12.1 & 3.6 & 2.8 & $\mathrm{~S}$ \\
\hline & & & & & & & OKALOOSA & COUNTY & & & & & \\
\hline 3( & $(024-636-1)$ & $\mathrm{F}$ & $800 \mathrm{R}$ & 500 & 1936 & B & $\begin{array}{r}+20.1 \\
1950\end{array}$ & $\begin{array}{c}-70.26 \\
1962\end{array}$ & -72.19 & -52.99 & 40.20 & 40.25 & $S$ \\
\hline 23 & $(034-626-1)$ & $\mathbf{F}$ & $652 \mathrm{R}$ & 409 & 1947 & B & $\begin{array}{r}-93.3 \\
1948\end{array}$ & $\begin{array}{r}-115.0 \\
1961\end{array}$ & -125.2 & $\cdots$ & $\cdots$ & $\cdots$ & $\mathrm{S}$ \\
\hline 25 & $(038-631-1)$ & $\mathbf{F}$ & $609 R$ & 456 & 1947 & B & $\begin{array}{r}-108.1 \\
1949\end{array}$ & $\begin{array}{r}-124.8 \\
1962\end{array}$ & -127.5 & -126.6 & 1.7 & 2.8 & $\mathrm{~S}$ \\
\hline 27 & $(030-635-2)$ & $\mathbf{F}$ & 591R & 422 & 1948 & B & $\begin{array}{r}-27.9 \\
1951\end{array}$ & $\begin{array}{r}-65.2 \\
1962\end{array}$ & -64.0 & $\cdots$ & 2.2 & 4.1 & $\mathrm{~S}$ \\
\hline 29 & $(035-637-1)$ & $\mathbf{F}$ & 766R & 524 & 1947 & B & $\begin{array}{r}-102.3 \\
1948\end{array}$ & $\begin{array}{r}-126.4 \\
1962\end{array}$ & -127.0 & -126.8 & 3.9 & 3.0 & $\mathrm{~S}$ \\
\hline 31 & $(037-645-1)$ & $\mathbf{F}$ & $690 \mathrm{R}$ & 527 & 1948 & B & $\begin{array}{r}-46.8 \\
1948\end{array}$ & $\begin{array}{r}-66.5 \\
1962\end{array}$ & -68.8 & -68.8 & 2.2 & 1.6 & $\mathrm{~s}$ \\
\hline 34 & $(028-629-1)$ & $\mathbf{F}$ & 540 & $\cdots$ & 1947 & B & $\begin{array}{r}+26.6 \\
1950\end{array}$ & $\begin{array}{l}-9.22 \\
1962\end{array}$ & -7.04 & -1.80 & 13.26 & 12.80 & $\mathbf{S}$ \\
\hline \multicolumn{14}{|c|}{ OKEECHOBEE COUNTY } \\
\hline 2 & & $\mathbf{S}$ & 21 & 18 & 1949 & $\mathrm{C}$ & $\begin{array}{r}+46.7 \\
1957\end{array}$ & $\begin{array}{c}+38.82 \\
1962\end{array}$ & +42.87 & +42.77 & 3.34 & 3.68 & M \\
\hline 3 & & $\mathbf{S}$ & 22 & 19 & 1948 & C & $\begin{array}{r}+61.3 \\
1959\end{array}$ & $\begin{array}{r}+56.7 \\
1950\end{array}$ & +59.98 & +60.60 & 3.60 & 2.99 & $\mathbf{M}$ \\
\hline & & & & & & & ORANGE C & COUNTY & & & & & \\
\hline 47 & $(832-128-1)$ & $\mathbf{F}$ & 350 & 328 & 1930 & C & $\begin{array}{l}-2.22 \\
1960\end{array}$ & $\begin{array}{c}-14.30 \\
1962\end{array}$ & -11.11 & -8.86 & 5.83 & 7.01 & \\
\hline $47 \mathrm{~B}$ & B $(832-128-3)$ & $\mathbf{S}$ & 17 & 17 & 1948 & B & $\begin{array}{l}+3.04 \\
1960\end{array}$ & $\begin{array}{c}-10.01 \\
1962\end{array}$ & -9.46 & -6.77 & 2.71 & 4.48 & \\
\hline $47 C$ & C $(832-128-4)$ & $\mathbf{S}$ & 50 & 46 & 1948 & B & $\begin{array}{c}-27.47 \\
1960\end{array}$ & $\begin{array}{c}-39.35 \\
1953\end{array}$ & -35.68 & -32.96 & 2.29 & 1.48 & \\
\hline 832 & $2-105-1$ & $\mathbf{F}$ & 492 & 151 & 1960 & $\mathbf{M}$ & $\begin{array}{c}-26.51 \\
1961\end{array}$ & $\begin{array}{c}-28.33 \\
1962\end{array}$ & -28.67 & -25.64 & 4.30 & 3.75 & E \\
\hline
\end{tabular}




\begin{tabular}{|c|c|c|c|c|c|c|c|c|c|c|c|c|}
\hline \multirow{4}{*}{ hit 11 ruaber } & \multirow{4}{*}{ 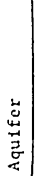 } & \multirow{4}{*}{ 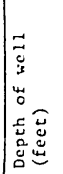 } & \multirow{4}{*}{ 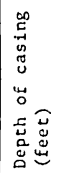 } & \multirow{4}{*}{ 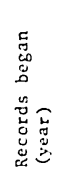 } & \multirow{4}{*}{ 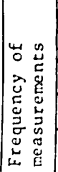 } & \multicolumn{6}{|c|}{$\begin{array}{l}\text { Water level above }(+) \text { or below }(-) \text { Land surface } \\
\text { (fect) }\end{array}$} & \multirow{4}{*}{ Remarks } \\
\hline & & & & & & \multirow{2}{*}{\multicolumn{2}{|c|}{$\begin{array}{l}\text { Prier to } 1963 \\
\text { May or June }\end{array}$}} & \multirow{2}{*}{\multicolumn{2}{|c|}{$\begin{array}{l}\text { Highest water } \\
\text { level in May } \\
\text { or June }\end{array}$}} & \multirow{2}{*}{\multicolumn{2}{|c|}{$\begin{array}{l}\text { Annual } \\
\text { Range }\end{array}$}} & \\
\hline & & & & & & & & & & & & \\
\hline & & & & & & $\begin{array}{c}\text { High } \\
\text { (year) }\end{array}$ & $\begin{array}{c}\text { Low } \\
\text { (year) }\end{array}$ & 1963 & 1964 & 1963 & 1964 & \\
\hline
\end{tabular}

\begin{tabular}{|c|c|c|c|c|c|c|c|c|c|c|c|c|}
\hline \multicolumn{13}{|c|}{ OSCEOLA COUNTY } \\
\hline 171 & $s$ & 19 & 13 & 1950 & c & $\begin{array}{r}+32.1 \\
1957\end{array}$ & $\begin{array}{r}+27.8 \\
1956\end{array}$ & +31.26 & +31.20 & 4.25 & 3.55 & 4 \\
\hline 179 & $\mathrm{~s}$ & 18 & 18 & 1949 & C & $\begin{array}{r}+47.1 \\
1960\end{array}$ & $\begin{array}{c}+43.27 \\
1962\end{array}$ & +46.82 & +46.90 & 3.18 & 3.15 & M \\
\hline 181 & $\mathrm{~s}$ & 15 & 14 & 1948 & C & $\begin{array}{r}+77.9 \\
1957\end{array}$ & $\begin{array}{c}+71.72 \\
1962\end{array}$ & +74.99 & +76.25 & 4.28 & 4.90 & M \\
\hline 182 & $\mathrm{~s}$ & 23 & 16 & 1949 & c & $\begin{array}{r}+61.3 \\
1957\end{array}$ & $\begin{array}{r}+56.7 \\
1950\end{array}$ & +61.02 & +60.86 & 3.17 & 3.65 & M \\
\hline 183 & $\mathrm{~s}$ & 27 & 22 & 1948 & c & $\begin{array}{r}+73.2 \\
1957\end{array}$ & $\begin{array}{r}+68.3 \\
1956\end{array}$ & +71.10 & +73.04 & 4.03 & 3.39 & M \\
\hline \multicolumn{13}{|c|}{ PALM BEACH COUNTY } \\
\hline 38 & B & 17 & 16 & 1944 & c & $\begin{array}{l}+8.6 \\
1948\end{array}$ & $\begin{array}{l}+3.6 \\
1956\end{array}$ & +5.80 & +6.36 & 4.18 & 4.60 & M \\
\hline 99 & B & 18 & 16 & 1948 & c & $\begin{array}{r}+10.0 \\
1957\end{array}$ & $\begin{array}{l}+5.5 \\
1956\end{array}$ & +7.07 & +8.15 & 4.28 & 4.33 & M \\
\hline $10 z$ & B & 37 & 12 & 1950 & c & $\begin{array}{c}+17.00 \\
1957\end{array}$ & $\begin{array}{c}+14.30 \\
1951\end{array}$ & +17.51 & --- & 2.6 & $-\cdot-$ & M; D, 1964 \\
\hline 109 & B & 14 & 9 & 1950 & C & $\begin{array}{r}+18.9 \\
1957\end{array}$ & $\begin{array}{r}+15.0 \\
1956\end{array}$ & +16.86 & +18.01 & 3.59 & 2.46 & M \\
\hline 110 & B & 8 & 8 & 1951 & c & $\begin{array}{l}-2.6 \\
1962\end{array}$ & $\begin{array}{l}-6.0 \\
1962\end{array}$ & -3.7 & -3.6 & 3.5 & 3.1 & B \\
\hline 436 & B & 12 & 11 & 1956 & c & $\begin{array}{l}-2.10 \\
1957\end{array}$ & $\begin{array}{l}-4.3 \\
1960\end{array}$ & -2.65 & $\cdots$ & 1.85 & $\cdots$ & B; D, 1964 \\
\hline \multicolumn{13}{|c|}{ PASCO COUNTY } \\
\hline $13(8 \pm 5-226-1)$ & $\mathbf{F}$ & 49 & 43 & 1934 & c & $\begin{array}{l}-4.77 \\
1959\end{array}$ & $\begin{array}{r}-10.1 \\
1945\end{array}$ & -7.20 & -6.41 & 3.69 & 4.15 & \\
\hline $826-2 \geq 1-i^{\prime}$ & $\mathbf{F}$ & 227 & 49 & 1959 & c & $\begin{array}{l}-9.97 \\
1960\end{array}$ & $\begin{array}{c}-22.75 \\
1962\end{array}$ & -18.76 & $\cdots$ & 4.19 & $\cdots$ & \\
\hline \multicolumn{13}{|c|}{ PINELLAS COUNTY } \\
\hline $13(808-245-1)$ & $\mathrm{F}$ & 141 & 33 & 1947 & c & $\begin{array}{l}-8.29 \\
1948\end{array}$ & $\begin{array}{c}-10.70 \\
1950\end{array}$ & -9.14 & -8.93 & 1.15 & 1.72 & T \\
\hline $77(804-245-1)$ & $\mathbf{F}$ & $282 \mathrm{R}$ & $\cdots$ & 1947 & c & $\begin{array}{l}-64.41 \\
1959860\end{array}$ & $\begin{array}{c}-68.01 \\
1949\end{array}$ & -65.69 & -- & 1.70 & $\cdots$ & D, 1964 \\
\hline $105(303-246-1)$ & $\mathbf{F}$ & 230 & 25 & 1947 & B & $\begin{array}{c}-26.56 \\
1959\end{array}$ & $\begin{array}{c}-29.53 \\
1962\end{array}$ & -28.35 & -- & 1.78 & -- & D, 1963 \\
\hline $166(306-247-1)$ & $\mathbf{F}$ & 195 & $\cdots$ & 1945 & B & $\begin{array}{c}-12.18 \\
1951\end{array}$ & $\begin{array}{c}-18.34 \\
1953\end{array}$ & -12.64 & -10.74 & 4.29 & 5.21 & \\
\hline $245(75 \varepsilon-247-1)$ & $\mathrm{F}$ & 208 & $\cdots$ & 1945 & c & $\begin{array}{c}-25.12 \\
1948\end{array}$ & $\begin{array}{c}-28.72 \\
1956\end{array}$ & -26.78 & -25.98 & 1.81 & 2.13 & $\mathrm{~T}$ \\
\hline $561(750-240-1)$ & $\mathbf{F}$ & 188 & $\cdots$ & 1947 & c & $\begin{array}{l}-1.53 \\
1948\end{array}$ & $\begin{array}{l}-4.24 \\
1962\end{array}$ & -3.6 & $\cdots$ & 2.12 & --- & D, 1964 \\
\hline $665(758-244-4)$ & $\mathbf{F}$ & 299 & 81 & 1954 & c & $\begin{array}{c}-20.12 \\
1959\end{array}$ & $\begin{array}{c}-24.55 \\
1955\end{array}$ & -21.58 & -21.31 & 1.61 & 2.30 & \\
\hline $667(755-243-1)$ & $\mathbf{F}$ & 845 & $\cdots$ & 1954 & C & $\begin{array}{c}-53.32 \\
1959\end{array}$ & $\begin{array}{c}-56.68 \\
1955\end{array}$ & -54.59 & -- & 1.73 & --- & D, 1964 \\
\hline
\end{tabular}




\begin{tabular}{|c|c|c|c|c|c|c|c|c|c|c|c|}
\hline \multirow{4}{*}{ We 11 number } & \multirow{4}{*}{ | } & \multirow{4}{*}{$\begin{array}{l}= \\
0 \\
3 \\
w \\
0 \\
5 \\
5 \\
0 \\
0 \\
0 \\
0\end{array}$} & \multirow{4}{*}{ 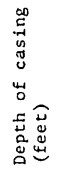 } & \multirow{4}{*}{ 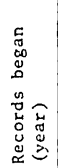 } & \multirow{4}{*}{ 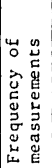 } & \multicolumn{5}{|c|}{$\begin{array}{c}\text { Water level above }(+) \text { or below (-) land surface } \\
\text { (feet) }\end{array}$} & \multirow{4}{*}{ Remarks } \\
\hline & & & & & & Prior to 1963 & \multirow{2}{*}{\multicolumn{2}{|c|}{$\begin{array}{c}\text { llighest water } \\
\text { level in May } \\
\text { or June }\end{array}$}} & \multirow{2}{*}{\multicolumn{2}{|c|}{$\begin{array}{l}\text { Annual } \\
\text { Range }\end{array}$}} & \\
\hline & & & & & & May or June & & & & & \\
\hline & & & & & & $\begin{array}{l}\text { Low } \\
\text { (year) }\end{array}$ & 1963 & 1964 & 1963 & 1964 & \\
\hline
\end{tabular}

\begin{tabular}{|c|c|c|c|c|c|c|c|c|c|c|c|c|c|}
\hline \multicolumn{14}{|c|}{ POLK COUNTY } \\
\hline 44 & $(810-136-1)$ & $\mathbf{F}$ & 195 & 81 & 1945 & $\mathrm{C}$ & $\begin{array}{l}-1.70 \\
1960\end{array}$ & $\begin{array}{l}-5.74 \\
1962\end{array}$ & -4.37 & -2.48 & 1.80 & 1.89 & \\
\hline 45 & $(759-158-1)$ & $\mathbf{F}$ & 643 & 325 & 1948 & M & $\begin{array}{c}-63.65 \\
1948\end{array}$ & $\begin{array}{c}-84.82 \\
1962\end{array}$ & -79.17 & -78.86 & 5.93 & 11.20 & $S$ \\
\hline 47 & $(810-136-2)$ & $\mathrm{S}$ & 67 & 60 & 1948 & C & $\begin{array}{r}+111.7 \\
1960\end{array}$ & $\begin{array}{r}+106.9 \\
1962\end{array}$ & +108.26 & +109.52 & 1.22 & 3.01 & M \\
\hline 48 & $(732-131-1)$ & S & 62 & 59 & 1949 & C & $\begin{array}{r}+100.8 \\
1954\end{array}$ & $\begin{array}{r}+96.2 \\
1956\end{array}$ & +96.97 & +97.45 & 0.84 & 2.41 & M \\
\hline 49 & $(748-119-1)$ & $\mathrm{S}$ & 17 & 14 & 1949 & $C$ & $\begin{array}{r}+104.7 \\
1957\end{array}$ & $\begin{array}{c}+98.99 \\
1962\end{array}$ & +100.99 & +101.80 & 3.72 & 3.50 & M \\
\hline 51 & $(744-131-1)$ & $\mathrm{H}$ & 319 & 208 & 1949 & C & $\begin{array}{l}-5.08 \\
1958\end{array}$ & $\begin{array}{c}-17.25 \\
1962\end{array}$ & -11.80 & -11.86 & 12.85 & 8.94 & $\mathrm{P}$ \\
\hline 753 & $3-158-311$ & $\mathrm{~F}$ & 710 & 237 & 1955 & $\mathrm{C}$ & $\begin{array}{c}-15.88 \\
1958\end{array}$ & $\begin{array}{c}-38.57 \\
1962\end{array}$ & -33.25 & -29.10 & 10.43 & 13.29 & $\mathrm{~S}$ \\
\hline 802 & $2-132-1$ & $\mathbf{F}$ & 463 & 137 & 1959 & B & --- & $\begin{array}{l}-7.65 \\
1961\end{array}$ & -11.68 & -10.15 & 1.97 & 1.99 & \\
\hline 805 & $5-155-2$ & F & 311 & 82 & 1956 & B & $\begin{array}{c}-15.18 \\
1959\end{array}$ & $\begin{array}{c}-25.64 \\
1962\end{array}$ & -21.07 & -19.56 & 4.95 & 3.92 & \\
\hline 805 & $5-155-3$ & $\mathrm{H}$ & 72 & 62 & 1955 & B & $\begin{array}{c}-12.52 \\
1959\end{array}$ & $\begin{array}{c}-21.73 \\
1962\end{array}$ & -18.15 & -16.52 & 4.24 & 3.64 & \\
\hline 806 & $5-156-1$ & $\mathrm{~S}$ & 11 & $8 *$ & 1955 & B & $\begin{array}{l}-3.69 \\
1959\end{array}$ & $\begin{array}{l}-8.86 \\
1962\end{array}$ & -9.73 & -7.91 & 1.35 & 3.38 & $\begin{array}{l}\text { * Scredned from } \\
8 \text { to } 11 \text { feet }\end{array}$ \\
\hline 806 & $6-156-2$ & $\mathrm{H}$ & 103 & 63 & 1956 & B & $\begin{array}{c}-16.89 \\
1959\end{array}$ & $\begin{array}{c}-29.66 \\
1962\end{array}$ & -23.58 & -23.99 & 5.09 & 4.65 & \\
\hline \multicolumn{14}{|c|}{ PUTNAM COUNTY } \\
\hline 28 & $(925-138-1)$ & $\mathbf{F}$ & 159 & -- & 1936 & B & $\begin{array}{l}-6.2 \\
1944\end{array}$ & $\begin{array}{l}-9.81 \\
1962\end{array}$ & -9.76 & -7.33 & 2.03 & 2.26 & \\
\hline 29 & $(939-138-1)$ & $\mathrm{F}$ & $300 R$ & -- & 1936 & B & $\begin{array}{l}+10.8 \\
1936847\end{array}$ & $\begin{array}{l}+2.02 \\
1962\end{array}$ & +2.37 & +5.92 & 2.93 & 2.90 & \\
\hline & $7-153-1$ & $\mathbf{F}$ & $303 R$ & 300 & 1934 & B & $\begin{array}{l}-29.51 \\
1961\end{array}$ & $\begin{array}{c}-35.65 \\
1957\end{array}$ & -32.74 & -30.26 & 1.12 & 1.68 & \\
\hline 939 & $9-134-11$ & $\mathbf{F}$ & 547 & 113 & 1958 & B & $\begin{array}{l}+4.26 \\
1959\end{array}$ & $\begin{array}{l}-1.75 \\
1962\end{array}$ & +0.06 & +2.10 & 6.80 & 1.31 & \\
\hline 943 & $3-152-1$ & $\mathrm{H}$ & 124 & $\cdots$ & 1956 & B & $\begin{array}{c}-43.20 \\
1961\end{array}$ & $\begin{array}{c}-46.66 \\
1957\end{array}$ & -46.18 & -44.14 & 1.86 & 2.46 & \\
\hline \multicolumn{14}{|c|}{ ST. JOHNS COUNTY } \\
\hline 5 & $(007-123-1)$ & $\mathbf{F}$ & 350R & 180 & 1934 & B & $\begin{array}{r}+43.9 \\
1951\end{array}$ & $\begin{array}{r}+35.0 \\
1962\end{array}$ & +33.8 & +38.0 & 2.2 & 5.0 & \\
\hline 8 & $(005-129-1)$ & $\mathbf{F}$ & $336 \mathrm{R}$ & 240 & 1934 & B & $\begin{array}{r}+36.5 \\
1947\end{array}$ & $\begin{array}{r}+23.3 \\
1962\end{array}$ & +22.7 & +24.3 & 1.2 & 1.8 & \\
\hline 9 & $(953-118-1)$ & $\mathbf{F}$ & 1,400 & 170 & 1930 & B & $\begin{array}{r}+34.2 \\
1947\end{array}$ & $\begin{array}{r}+19.5 \\
1962\end{array}$ & -- & $-\infty$ & $\cdots$ & $\cdots$ & D, 1962 \\
\hline & $0-123-2$ & $\mathbf{F}$ & 258 & -- & 1957 & B & $\begin{array}{l}+4.72 \\
1959\end{array}$ & $\begin{array}{l}-0.57 \\
1962\end{array}$ & +0.27 & +2.66 & 4.65 & 2.01 & \\
\hline & $7-122-1$ & $\mathbf{F}$ & 622 & 142 & 1958 & C & $\begin{array}{c}-17.30 \\
1959\end{array}$ & $\begin{array}{c}-21.68 \\
1962\end{array}$ & -21.51 & -19.10 & 3.30 & 3.02 & \\
\hline
\end{tabular}




\begin{tabular}{|c|c|c|c|c|c|c|c|c|c|c|c|c|}
\hline \multirow{4}{*}{ We 11 number } & \multirow{4}{*}{. } & \multirow{4}{*}{ 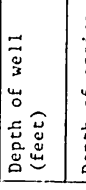 } & \multirow{4}{*}{ 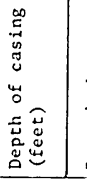 } & \multirow{4}{*}{ 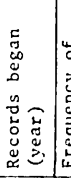 } & \multirow{4}{*}{ 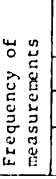 } & \multicolumn{6}{|c|}{$\begin{array}{c}\text { Water level above }(+) \text { or below }(-) \text { land surface } \\
\text { (feet) }\end{array}$} & \multirow{4}{*}{ Remarks } \\
\hline & & & & & & \multirow{2}{*}{\multicolumn{2}{|c|}{$\begin{array}{l}\text { Prior to } 1963 \\
\text { May or June }\end{array}$}} & \multirow{2}{*}{\multicolumn{2}{|c|}{$\begin{array}{l}\text { llighest water } \\
\text { level in May } \\
\text { or June }\end{array}$}} & \multirow{2}{*}{\multicolumn{2}{|c|}{$\begin{array}{l}\text { Annual } \\
\text { Range }\end{array}$}} & \\
\hline & & & & & & & & & & & & \\
\hline & & & & & & $\begin{array}{l}\text { High } \\
(\text { year })\end{array}$ & $\begin{array}{l}\text { Low } \\
(\text { year })\end{array}$ & 1963 & 1964 & 1963 & 1964 & \\
\hline $941-129-7$ & $\mathbf{F}$ & 541 & --- & 1955 & B & $\begin{array}{r}+10.1 \\
1959\end{array}$ & $\begin{array}{l}+1.52 \\
1962\end{array}$ & +3.69 & +5.6 & 12.25 & 3.00 & $\mathbf{P}$ \\
\hline $947-126-1$ & $\mathbf{F}$ & 275 & $\cdots$ & 1956 & B & $\begin{array}{l}-1.55 \\
1958\end{array}$ & $\begin{array}{l}-10.86 \\
1962\end{array}$ & -6.99 & -6.23 & 20.04 & 2.96 & $\mathbf{P}$ \\
\hline \multicolumn{13}{|c|}{ ST. LUCIE COUNTY } \\
\hline 41 & $\mathrm{~s}$ & 17 & 13 & 1950 & C & $\begin{array}{r}+28.2 \\
1957\end{array}$ & $\begin{array}{r}+25.2 \\
1956\end{array}$ & +27.56 & +26.86 & 2.41 & 3.17 & $\mathbf{M}$ \\
\hline 42 & $\mathrm{~s}$ & 18 & 13 & 1950 & $\mathrm{C}$ & $\begin{array}{r}+26.9 \\
1951\end{array}$ & $\begin{array}{c}+23.76 \\
1961\end{array}$ & +25.06 & +25.12 & 3.97 & 3.59 & M \\
\hline \multicolumn{13}{|c|}{ SANTA ROSA COUNTY } \\
\hline $10(032-648-1)$ & G & $197 R$ & 140 & 1947 & B & $\begin{array}{r}-80.1 \\
1948\end{array}$ & $\begin{array}{r}-91.3 \\
1957\end{array}$ & -87.7 & -85.5 & 1.7 & 5.1 & \\
\hline $102(021-709-8)$ & $\mathrm{s}$ & 41 & $31 *$ & 1950 & $\mathrm{c}$ & $\begin{array}{l}-4.43 \\
1960\end{array}$ & $\begin{array}{l}-9.52 \\
1955\end{array}$ & -7.26 & -4.00 & 2.39 & 4.41 & $\begin{array}{l}\text { *Screened from } \\
31 \text { to } 41 \text { feet }\end{array}$ \\
\hline $035-706-1$ & G & 211 & $206 *$ & 1959 & M & $\begin{array}{c}-82.84 \\
1961\end{array}$ & $\begin{array}{c}-85.77 \\
1959\end{array}$ & -89.10 & -85.94 & 1.84 & 5.85 & $\begin{array}{l}\text { *Screened from } \\
206 \text { to } 211 \text { feet }\end{array}$ \\
\hline $040-708-1$ & G & 128 & $123 *$ & 1959 & M & $\begin{array}{l}+4.83 \\
1961\end{array}$ & $\begin{array}{l}+2.11 \\
1959\end{array}$ & +1.28 & +4.00 & 1.57 & 3.26 & $\begin{array}{l}\text { *Screened from } \\
123 \text { to } 128 \text { feet }\end{array}$ \\
\hline $041-649-1$ & $\mathbf{G}$ & 98 & $93+$ & 1959 & B & $\begin{array}{c}-56.34 \\
1960\end{array}$ & $\begin{array}{c}-59.72 \\
1961\end{array}$ & -61.90 & -61.30 & 2.62 & 6.20 & $\begin{array}{l}\text { *Screened from } \\
93 \text { to } 98 \text { feet }\end{array}$ \\
\hline \multicolumn{13}{|c|}{ SARASOTA COUNTY } \\
\hline $9(719-225-1)$ & $\mathbf{F}$ & $730 \mathrm{R}$ & 101 & 1930 & C & $\begin{array}{l}+4.51 \\
1931\end{array}$ & $\begin{array}{l}-9.36 \\
1962\end{array}$ & -7.88 & -3.10 & 8.78 & 5.12 & $\mathrm{~s}$ \\
\hline \multicolumn{13}{|c|}{ SEMINOLE COUNTY } \\
\hline $125(841-122-1)$ & $\mathbf{F}$ & 158 & 74 & 1951 & C & $\begin{array}{c}-34.18 \\
1960\end{array}$ & $\begin{array}{c}-42.60 \\
1962\end{array}$ & $\cdots$ & -38.79 & 2.83 & 4.43 & \\
\hline $257(847-113-6)$ & $\mathbf{F}$ & 206 & $\cdots$ & 1951 & B & $\begin{array}{l}+5.10 \\
1953\end{array}$ & $\begin{array}{l}+0.27 \\
1962\end{array}$ & +1.09 & +3.10 & 3.11 & 2.92 & \\
\hline \multicolumn{13}{|c|}{ SUMTER COUNTY } \\
\hline $852-201-1$ & $\mathbf{F}$ & 125 & 45 & 1961 & B & $\cdots$ & -- & -33.26 & -29.94 & 2.42 & 10.13 & \\
\hline \multicolumn{13}{|c|}{ SUWANNEE COUNTY } \\
\hline $019-249-1$ & $\mathbf{F}$ & 138 & 135 & 1961 & B & $\cdots$ & $\begin{array}{c}-33.02 \\
1962\end{array}$ & -35.31 & -18.94 & 4.03 & 18.07 & \\
\hline \multicolumn{13}{|c|}{ TAYLOR COUNTY } \\
\hline $35(003-330-1)$ & $\mathbf{F}$ & 245 & 189 & 1946 & C & $\begin{array}{l}-1.00 \\
1949\end{array}$ & $\begin{array}{r}-30.9 \\
1962\end{array}$ & -24.0 & -16.2 & 7.1 & 20.63 & $\mathbf{P}$ \\
\hline $36(004-331-1)$ & $\mathbf{S}$ & 35 & $\cdots$ & 1947 & C & $\begin{array}{l}-5.10 \\
1948\end{array}$ & $\begin{array}{c}-23.95 \\
1957\end{array}$ & -11.06 & -5.05 & 4.81 & 10.77 & $\mathbf{P}$ \\
\hline \multicolumn{13}{|c|}{ UNION COUNTY } \\
\hline $001-224-1$ & $\mathbf{F}$ & 256 & 198 & 1959 & B & $\begin{array}{c}-89.54 \\
1961\end{array}$ & $\begin{array}{c}-92.57 \\
1962\end{array}$ & -93.57 & -90.73 & 1.10 & 5.67 & \\
\hline $007-222-1$ & $\mathbf{P}$ & 724 & 694 & 1958 & $\mathrm{C}$ & $\begin{array}{c}-86.92 \\
1959\end{array}$ & $\begin{array}{c}-93.00 \\
1962\end{array}$ & -92.69 & -89.52 & 2.72 & 6.69 & \\
\hline
\end{tabular}




\begin{tabular}{|c|c|c|c|c|c|c|c|c|c|c|c|c|}
\hline \multirow{4}{*}{ We 11 number } & \multirow{4}{*}{ | } & \multirow{4}{*}{ 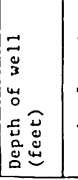 } & \multirow{4}{*}{ 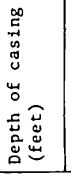 } & \multirow{4}{*}{ 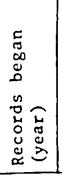 } & \multirow{4}{*}{ 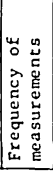 } & \multicolumn{6}{|c|}{$\begin{array}{l}\text { Water level above }(+) \text { or below }(-) \text { land surface } \\
(\text { feet) }\end{array}$} & \multirow{4}{*}{ Remarks } \\
\hline & & & & & & \multirow{2}{*}{\multicolumn{2}{|c|}{$\frac{\text { Prior to } 1963}{\text { May or June }}$}} & \multirow{2}{*}{\multicolumn{2}{|c|}{$\begin{array}{c}\text { Highest water } \\
\text { level in May } \\
\text { or June }\end{array}$}} & \multirow{2}{*}{\multicolumn{2}{|c|}{$\begin{array}{l}\text { Annual } \\
\text { Range }\end{array}$}} & \\
\hline & & & & & & & & & & & & \\
\hline & & & & & & $\begin{array}{l}\text { High } \\
\text { (year) }\end{array}$ & $\begin{array}{l}\text { Low } \\
\text { (year) }\end{array}$ & 1963 & 1964 & 1963 & 1964 & \\
\hline \multicolumn{13}{|c|}{ VOLUSIA COUNTY } \\
\hline $29(911-125-1)$ & $\mathrm{F}$ & 107 & $\cdots$ & 1936 & B & $\begin{array}{l}-11.86 \\
1951\end{array}$ & $\begin{array}{c}-18.57 \\
1962\end{array}$ & -18.73 & -16.69 & 1.76 & 2.05 & \\
\hline $30(917-128-1)$ & $\mathbf{F}$ & $180 \mathrm{R}$ & -- & 1936 & B & $\begin{array}{r}+11.2 \\
1959\end{array}$ & $\begin{array}{l}+6.7 \\
1948\end{array}$ & +8.2 & +10.0 & 1.8 & 2.2 & \\
\hline $31(856-105-1)$ & $\mathbf{F}$ & 113 & $\cdots$ & 1936 & c & $\begin{array}{l}-4.72 \\
1953\end{array}$ & $\begin{array}{l}-8.60 \\
1962\end{array}$ & -6.83 & -6.05 & 2.58 & 3.33 & \\
\hline $32(919-125-1)$ & $\mathbf{F}$ & $138 \mathrm{R}$ & -- & 1936 & B & $\begin{array}{l}-1.2 \\
1937 \& 38\end{array}$ & $\begin{array}{l}-4.94 \\
1962\end{array}$ & -5.11 & -2.86 & 2.24 & 2.09 & \\
\hline $905-113-3$ & $\mathrm{~F}$ & 351 & 93 & 1955 & c & $\begin{array}{l}-0.22 \\
1958\end{array}$ & $\begin{array}{l}-3.66 \\
1956\end{array}$ & -1.74 & -0.70 & 2.56 & 3.27 & \\
\hline $909-106-1$ & $\mathbf{F}$ & 235 & 102 & 1955 & B & $\begin{array}{l}-5.25 \\
1959\end{array}$ & $\begin{array}{l}-5.87 \\
1955\end{array}$ & -8.07 & -6.25 & 2.90 & 2.37 & \\
\hline $909-106-4$ & $\vec{r}$ & 234 & 102 & 1955 & C & $\begin{array}{l}-4.95 \\
1958\end{array}$ & $\begin{array}{c}-10.21 \\
1962\end{array}$ & -7.15 & -5.56 & 3.85 & 4.38 & \\
\hline $909-106-9$ & $\mathrm{~F}$ & 496 & 480 & 1955 & B & $\begin{array}{l}-6.62 \\
1958\end{array}$ & $\begin{array}{l}-7.18 \\
1960\end{array}$ & -9.55 & -7.71 & 2.33 & 1.86 & \\
\hline $910-105-1$ & $\mathbf{F}$ & 220 & 152 & 1955 & C & $\begin{array}{c}-12.84 \\
1958\end{array}$ & $\begin{array}{c}-19.73 \\
1962\end{array}$ & -15.36 & -13.53 & 5.48 & 7.23 & \\
\hline $911-104-4$ & $\mathrm{~F}$ & 235 & 115 & 1955 & B & $\begin{array}{c}-15.72 \\
1955\end{array}$ & $\begin{array}{c}-20.81 \\
1958\end{array}$ & -25.85 & -21.82 & 7.27 & 9.75 & \\
\hline $911-104-9$ & $\mathbf{F}$ & 500 & 483 & 1955 & B & $\begin{array}{c}-10.26 \\
1948\end{array}$ & $\begin{array}{c}-12.63 \\
1956\end{array}$ & -13.89 & -11.85 & 2.32 & 2.20 & \\
\hline \multicolumn{13}{|c|}{ WAKULLA COUNTY } \\
\hline $2(009-412-1)$ & $\mathrm{F}$ & 65 & 22 & 1937 & B & $\begin{array}{l}-0.86 \\
1958\end{array}$ & $\begin{array}{l}-3.05 \\
1951\end{array}$ & -2.15 & -1.42 & 0.96 & 1.68 & $\mathrm{~T}$ \\
\hline $11(000-426-1)$ & F & 70 & 45 & 1946 & B & $\begin{array}{l}-5.58 \\
1955\end{array}$ & $\begin{array}{l}-8.25 \\
1960\end{array}$ & -5.70 & -6.90 & 1.83 & 0.39 & $\mathrm{~T}$ \\
\hline $005-417-1$ & $\mathrm{~F}$ & 77 & -- & 1961 & B & $\begin{array}{l}-2.02 \\
1961\end{array}$ & $\begin{array}{l}-2.43 \\
1962\end{array}$ & -3.48 & -1.13 & 2.40 & 1.03 & \\
\hline $011-410-1$ & $\mathrm{~F}$ & 80 & -- & 1961 & B & $\begin{array}{l}-0.96 \\
1961\end{array}$ & $\begin{array}{l}-1.87 \\
1962\end{array}$ & -1.72 & -0.12 & 1.48 & 1.63 & \\
\hline \multicolumn{13}{|c|}{ WALTON COUNTY } \\
\hline $13(022-606-1)$ & $\mathrm{F}$ & $450 R$ & $\cdots$ & 1936 & B & $\begin{array}{r}+15.8 \\
1950\end{array}$ & $\begin{array}{r}+11.1 \\
1956\end{array}$ & +10.6 & +13.1 & 0.5 & 2.3 & \\
\hline $17(029-607-2)$ & $\mathrm{F}$ & $187 \mathrm{R}$ & -- & 1947 & B & $\begin{array}{r}+30.7 \\
1948\end{array}$ & $\begin{array}{r}+25.4 \\
1957\end{array}$ & +23.9 & $\cdots$ & 0.8 & -- & D, 1964 \\
\hline $019-610-1$ & $\mathbf{F}$ & 615 & 188 & 1961 & B & $-\cdots$ & $\begin{array}{r}+12.5 \\
1962\end{array}$ & +11.6 & +14.7 & 1.1 & 2.8 & \\
\hline $029-614-1$ & $\mathrm{~F}$ & 160 & -- & 1961 & B & $\cdots$ & $\begin{array}{r}+20.5 \\
1962\end{array}$ & +19.5 & +21.0 & 0.6 & 2.0 & \\
\hline $043-612-1$ & F & 509 & 323 & 1961 & B & -- & $\begin{array}{r}-148.2 \\
1962\end{array}$ & -146.0 & -144.2 & 1.2 & 1.4 & \\
\hline & & & & & & WASHINGTOI & N COUNTY & & & & & \\
\hline $4(046-548-1)$ & $\mathbf{F}$ & $785 R$ & $-\cdots$ & 1935 & B & $\begin{array}{l}-9.47 \\
1953\end{array}$ & $\begin{array}{c}-15.09 \\
1954\end{array}$ & -14.05 & -7.20 & 3.71 & 5.26 & \\
\hline $037-542-2$ & $\mathbf{F}$ & 206 & 202 & 1961 & B & $\cdots$ & $\begin{array}{c}-19.65 \\
1962\end{array}$ & -20.20 & -13.72 & 2.07 & 4.31 & \\
\hline
\end{tabular}


period constitute a base for comparison from year to year. Highest and lowest water levels of record for May or June prior to 1963 are given in the table. Generally, highest and lowest levels are highest daily levels if taken from recorder charts. The range of fluctuations for 1963 and 1964 are shown under "Annual range"

\section{WELL-NUMBERING SYSTEM}

Two well-numbering systems are used in this report. Observation wells in Florida are numbered serially by counties and/or by a grid-coordinate system on latitude and longitude of the well location. Frequently, both numbers are assigned to a well e.g. a well number may be shown as 20(008-537-2). This affords a tie-in with water-level data published previously under well number 20 in Bay County with data that may be published for the same well under number 008-537-2.

Some wells used in table 1 have numbers with a letter prefix or suffix. In Broward and Dade counties, the letter prefixes G, S, F, and NP denote Geological Survey wells, supply wells, fire wells, and National Park Service wells, respectively. In Dade, Escambia, Highlands, Holmes, and Leon counties, the letter suffix A denotes a shallow well near a deeper well having the corresponding number without the letter suffix. In Orange County, the letter suffixes $\mathrm{B}$ and $\mathrm{C}$ denote shallow wells drilled in the vicinity of well 47 .

The grid-coordinate well-numbering system in Florida is derived from latitude and longitude coordinates. The latitude and longitude prefix north and west and the first digit of the degree number are not included in the well number.

The well number is a composite of three numbers separated by hyphens: the first number is composed of the last digit of the degree and the two digits of the minute that define the latitude on the south side of the 1-minute quadrangle; the second number is composed of the last digit of the degree and two digits of the minutes that define the longitude on the east side of a 1-minute quadrangle; and, the third number gives the numerical order in which the well was inventoried in the 1-minute quadrangle. For example, well number $835-105-1$ is the first well inventoried in the 1-minute quadrangle north of the $28^{\circ} 35^{\prime}$ parallel of latitude and west of the $81^{\circ} 05^{\prime}$ meridian of longitude. The location of well $835-105-1$ is shown diagrammatically in figure 2 . 

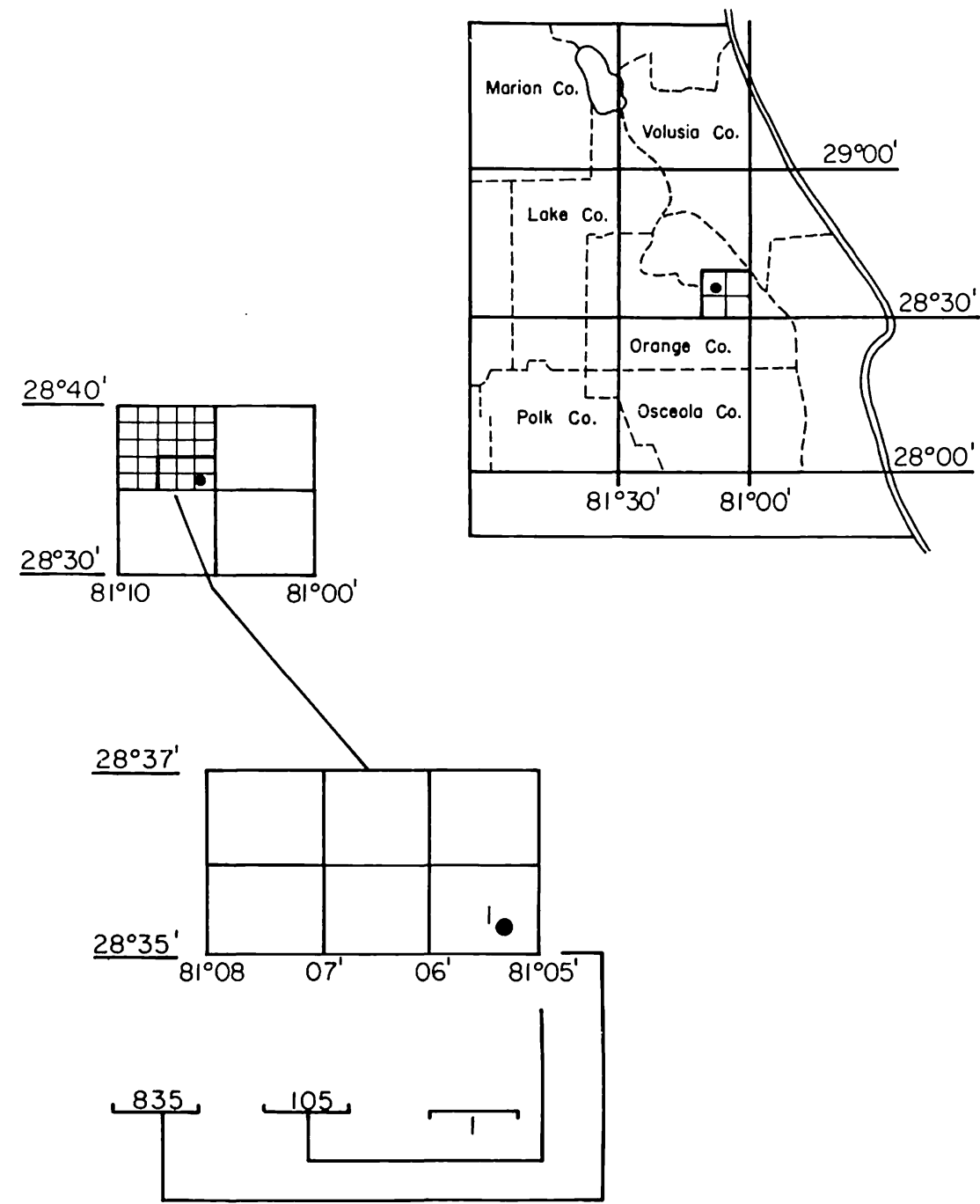

Figure 2. Well-numbering system. 


\section{PRINCIPAL AQUIFERS}

Ground-water supplies for industrial, agricultural, and municipal use in Florida are available from three principal aquifers: the Floridan aquifer in central and northern Florida; the Biscayne aquifer in southeastern Florida; and, the sand-and-gravel aquifer in the extreme northwestern part of Florida. The generalized areal extent of the aquifers supplying most of the ground water is shown in figure 1.

The Floridan aquifer, which underlies all of the State, is the principal source of water in central, northern, and most of northwestern Florida, as shown on figure 3. Highly mineralized

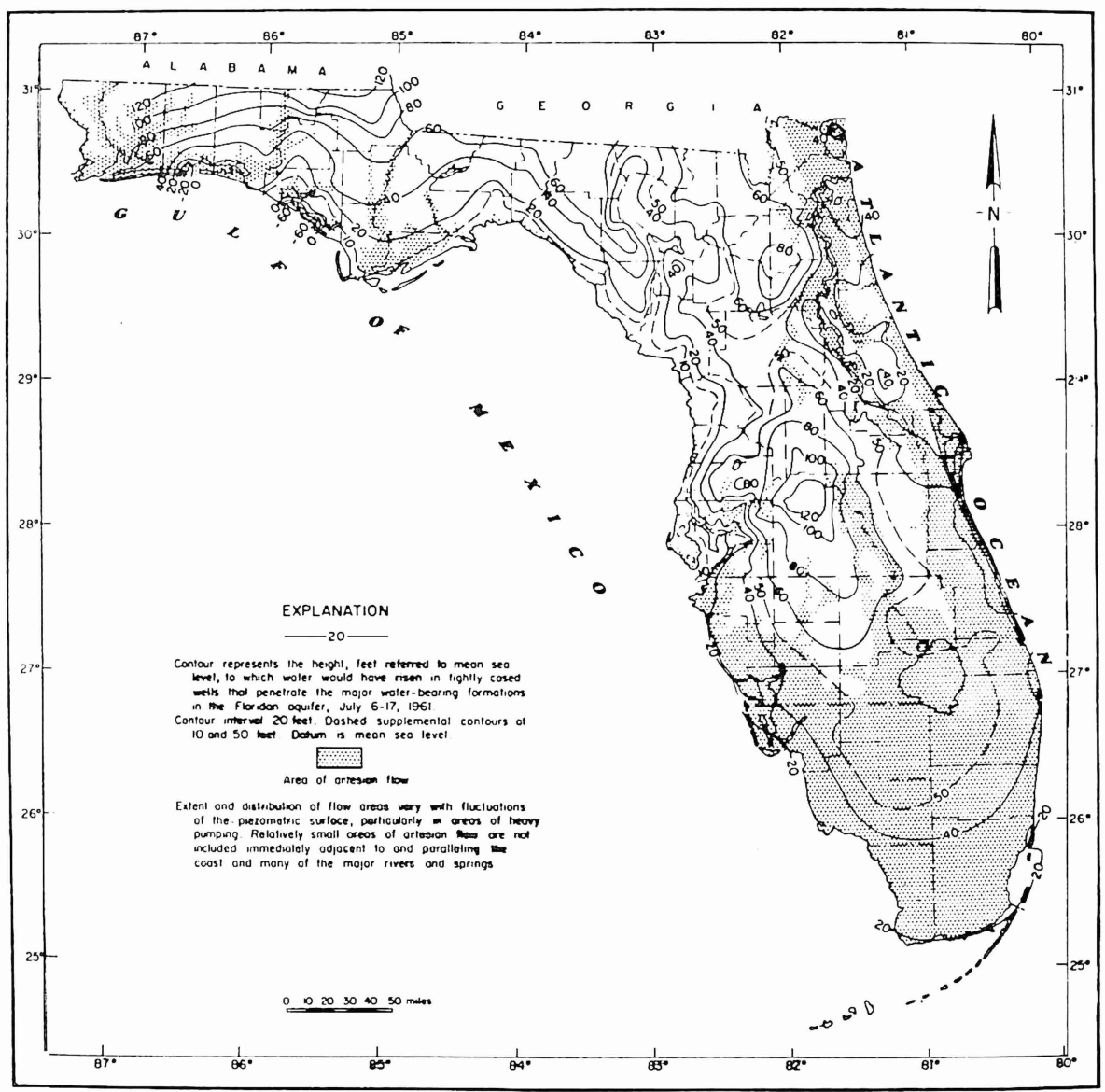

Figure 3. Map showing piezometric surface and areas of 'flow of the Floridan Aquifer, in Florida, July 6-17, 1961. 
water precludes the usefulness of the Floridan aquifer as a source of potable water in some coastal areas and in most of southern Florida. In those areas, shallow artesian and nonartesian aquifers are the source of supply.

The Biscayne aquifer in southeastern Florida is the chief source of water supply for industries, municipalities, and irrigation. This aquifer, one of the most highly productive aquifers in the world, underlies about 3,500 square miles of Broward, Dade, and Palm Beach counties. The usefulness of the Biscayne aquifer is sharply restricted in areas adjacent to the coast because of the presence of saline waters.

The sand-and-gravel aquifer in extreme northwestern Florida is the principal source of water supply, yielding large quantities of water for industries and municipalities. The aquifer underlies all of Escambia and Santa Rosa counties and part of western Okaloosa County.

This report of ground-water conditions has been divided into four geographical areas as follows: (1) northwestern Florida, (2) northern, northeastern, and north-central Florida; (3) central Florida; and, (4) southern and southeastern coastal Florida.

\section{NORTHWESTERN FLORIDA}

Northwestern Florida as used here includes the Panhandle region extending from the Apalachicola River westward to the Florida-Alabama line, figure 4.

The principal sources of ground-water supply in the region are the sand-and-gravel aquifer in Escambia and Santa Rosa counties and the Floridan aquifer in the rest of the region. Minor supplies of ground water are obtained from shallow nonartesian aquifers.

The Florida Panhandle includes three rapidly growing areas of industry and population: the Pensacola area, the Ft. Walton Beach area, the Panama City area.

\section{PENSACOLA AREA}

The Pensacola area includes Escambia and Santa Rosa counties. This area, like many others in the State, is undergoing rapid economic development. Industrial and municipal water uses are increasing. Pumpage in the Pensacola area in 1964 was about five times that in 1940. Figure 5 shows pumpage for the City of Pensacola, 1933-64. 


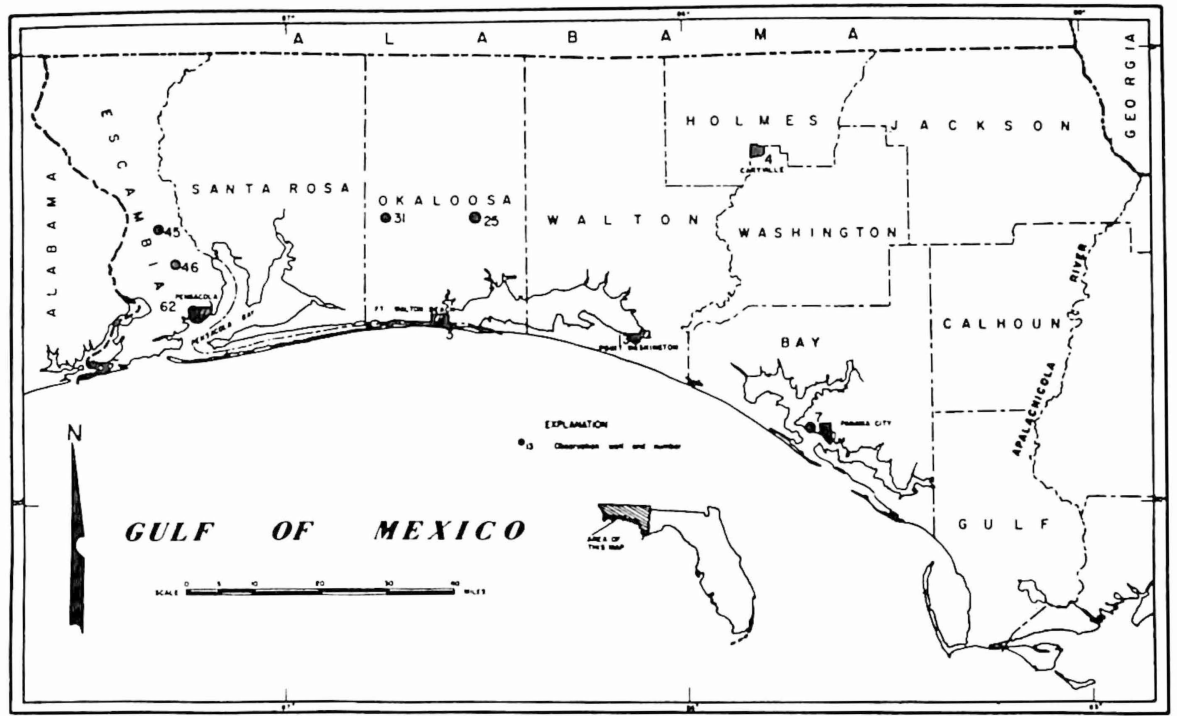

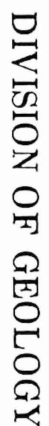

Figure 4. Locations of observation wells in northwestern Florida for which hydrographs are given. 


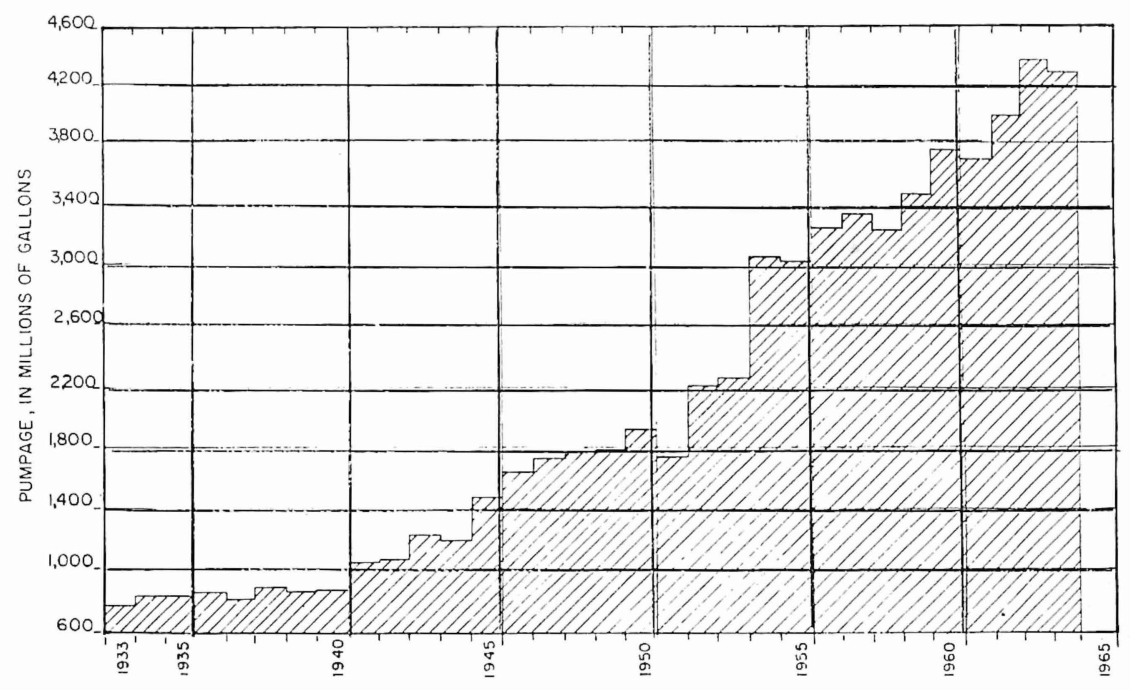

Figure 5. Graph of total yearly pumpage, City of Pensacola.

The observation-well program in the Pensacola area began in 1939 as part of the investigation to determine the adequacy and permanency of ground-water supply in Escambia County. Figure 4 shows locations of the observation wells selected from the hydrologic-data network for which hydrographs are given in this report, and table 1 presents data on 13 wells in Escambia County and 5 wells in Santa Rosa County. Figure 6 shows fluctuations and long-term trends of artesian water levels in the sand-and-gravel aquifer in the Pensacola area from 1940 through 1964.

Comparison of the hydrographs for the period of record reveals that while water levels at the end of 1964 declined in central Escambia County, water levels in the southern part of the county near the coast were above 1940 levels. Declines of artesian water levels in the sand-and-gravel aquifer ranged from a maximum of nearly 35 feet in well Escambia 45 at Cantonment to a minimum of less than 2 feet in well Escambia 46 near Ensley during 194164 .

In the coastal area, at Pensacola, the artesian water level in well Escambia 62, at the end of 1964, was about 2 feet above the 1942 level. The trends and fluctuations of artesian groundwater levels in well Escambia 62 and departures from monthly average rainfall at Pensacola, 1960-64 are shown in figure 7 . 

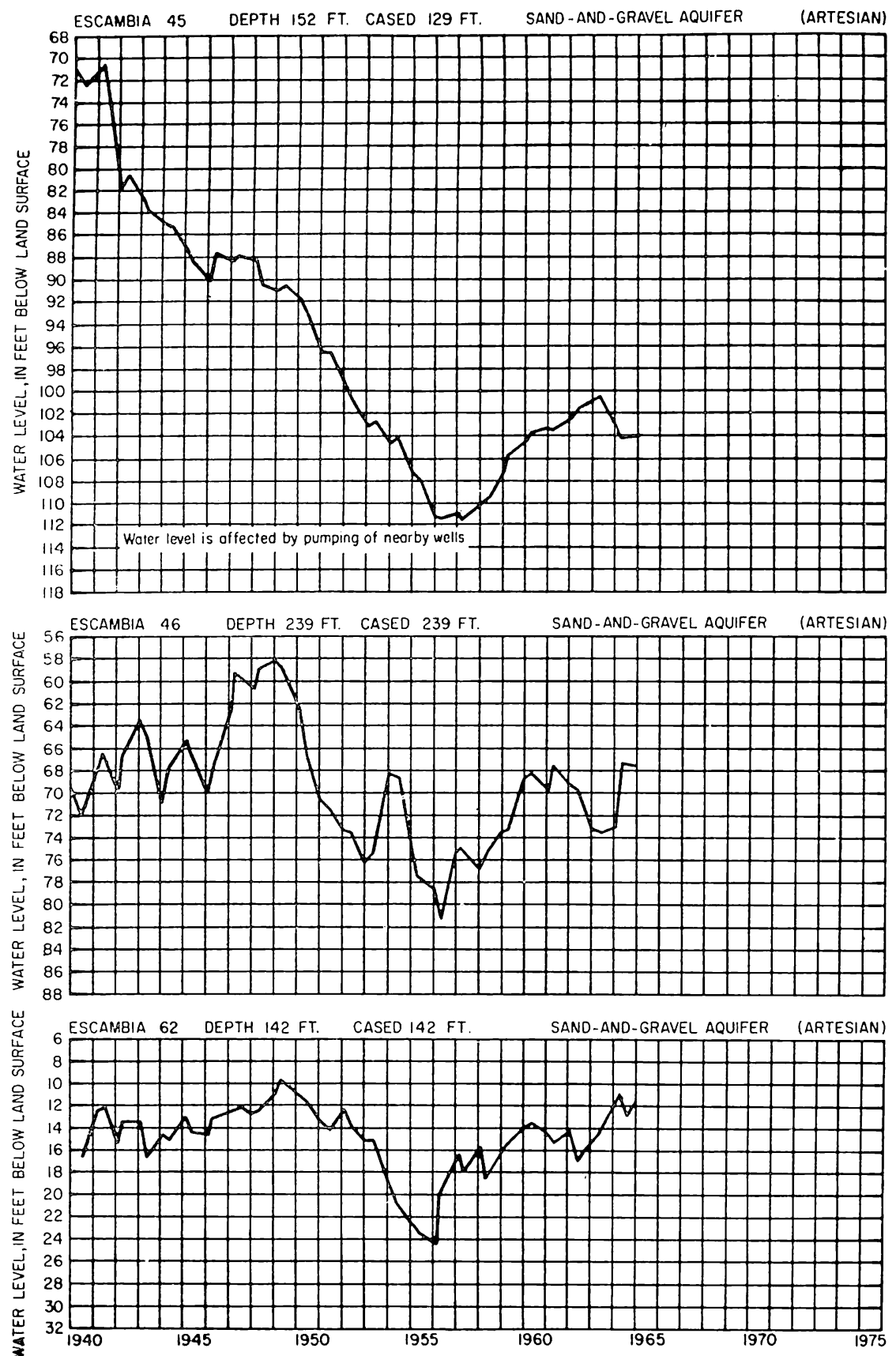

Figure 6. Hydrographs showing trends and fluctuations of water levels in wells Escambia 45 at Cantonment, 46 near Ensley, and 62 at Pensacola, Pensacola area. 

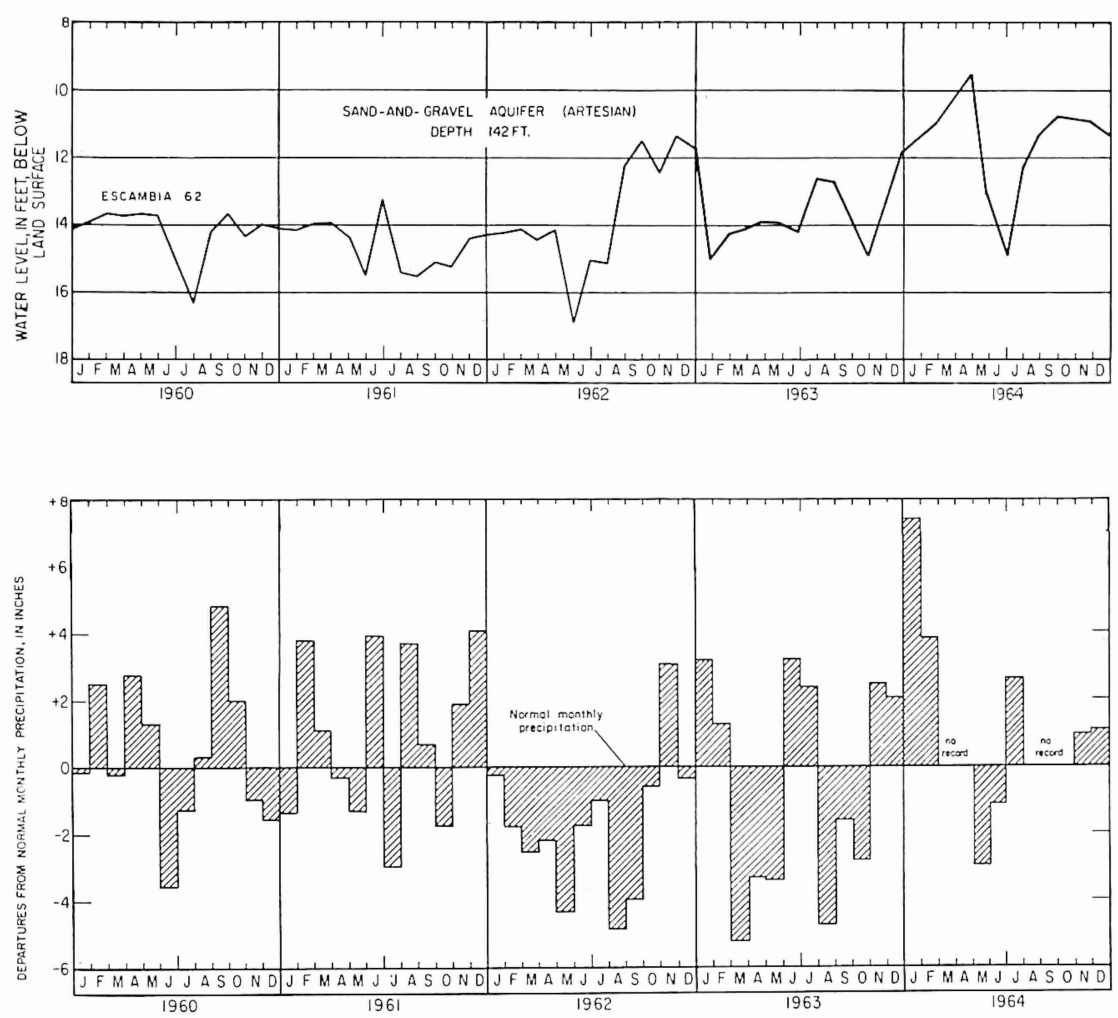

Figure 7. Hydrographs showing trends and fluctuations of water levels in well Escambia 62 and departures from monthly normal precipitation at Pensacola, 1960-64.

FT. WALTON AREA

The Ft. Walton area includes the Ft. Walton Beach area and Eglin Air Force Base at Niceville. The rate of growth of industry and population is accelerating. Pumpage from the Floridan aquifer for all uses is about $10.0 \mathrm{mgd}$ (million gallons per day). As a result of continuing heavy pumping, water levels have declined in about a 640 square-mile area.

The hydrograph of well Okaloosa 3 at Ft. Walton Beach, figure 8 , shows a maximum decline of 98.3 feet from 18.5 feet above land surface in 1947 to 79.8 feet below land surface in 1964. In August 1936, the artesian water level was 46 feet above land-surface datum. During the period from August 1936 to July 

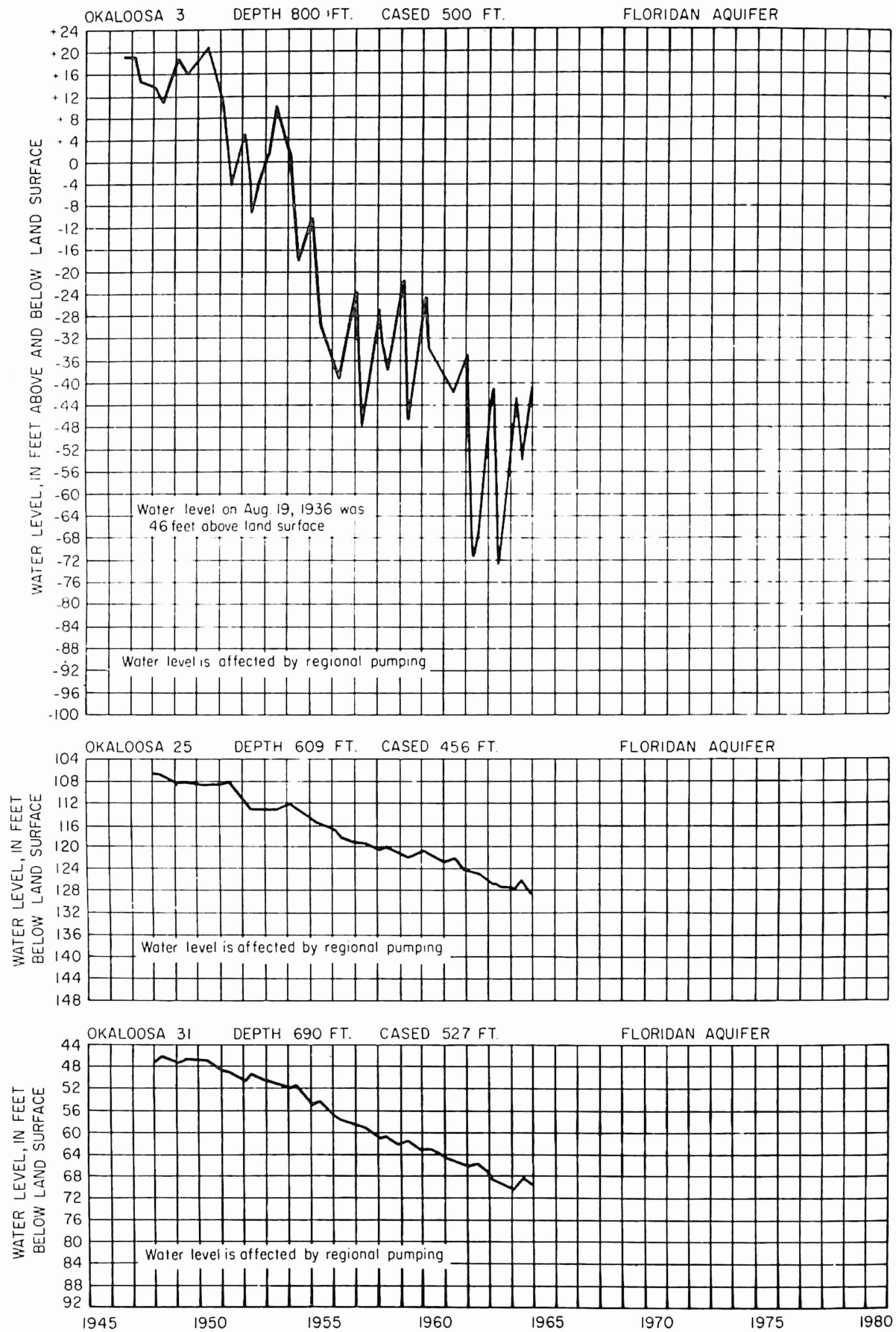

Figure 8. Hydrographs showing trends and fluctuations of water levels in wells Okaloosa 3, 25, and 31, Ft. Walton Beach area. 
1964, the water level in well Okaloosa 3 declined 125 feet, from 46 feet above land surface to 79 feet below land surface. The areal extent of the decline in artesian levels in the vicinity of Ft. Walton Beach is shown by the net change of water levels map, figures 9 and 10. Water-level changes during 1951-62 are shown by figure 9. Changes of groundwater levels for the current period May 1962-64 are shown by figure 10 .

\section{PANAMA CITY AREA}

The Panama City area includes 250 square miles in Bay County, including Tyndall Air Force Base.

The Floridan aquifer supplies most of the water for municipal, industrial, and military needs in the area. Figure 11 shows total pumpage from the Panama City well fields at St. Andrews and Millville for the period 1944-64. Pumpage of ground water for municipal use declined from an average of $1,200 \mathrm{mgy}$ (million gallons per year) in 1961-62 to an average of $860 \mathrm{mgy}$ in 1963-64. To some extent, reduced pumpage by Panama City and a change in locale of source of water by the pulp industry allowed water levels to rise sharply during 1963-64. Instead of the usual fall and winter rise of 2 to 7 feet, water levels rose 24 feet from 78 feet to 54 feet below land surface from June 1963 through December 1964. The alteration of the pattern of fluctuations of water levels was probably also the result of above average annual rainfall in the area during 1964.

The long-term trends and fluctuations of ground-water levels at Panama City are shown by the hydrograph of well Bay 7 , figure 12. The decline of water level in well Bay 7 represents the maximum known decline in the area and is caused by pumping in nearby wells. In August 1936, the water level in well Bay 7 was about 36 feet below land surface, while in June 1963, it was about 78 feet below land surface, a maximum decline of 42 feet.

\section{NORTHERN AND NORTH-CENTRAL FLORIDA}

Northern and north-central Florida as used in this report extends from the Apalachicola River eastward to the Atlantic Ocean and from the Georgia line southward to the latitude of Ocala, figure 13, and includes 24 counties and parts of Levy Marion, and Volusia counties. The Floridan aquifer is the principal source of ground-water supply in areas along the coast in 


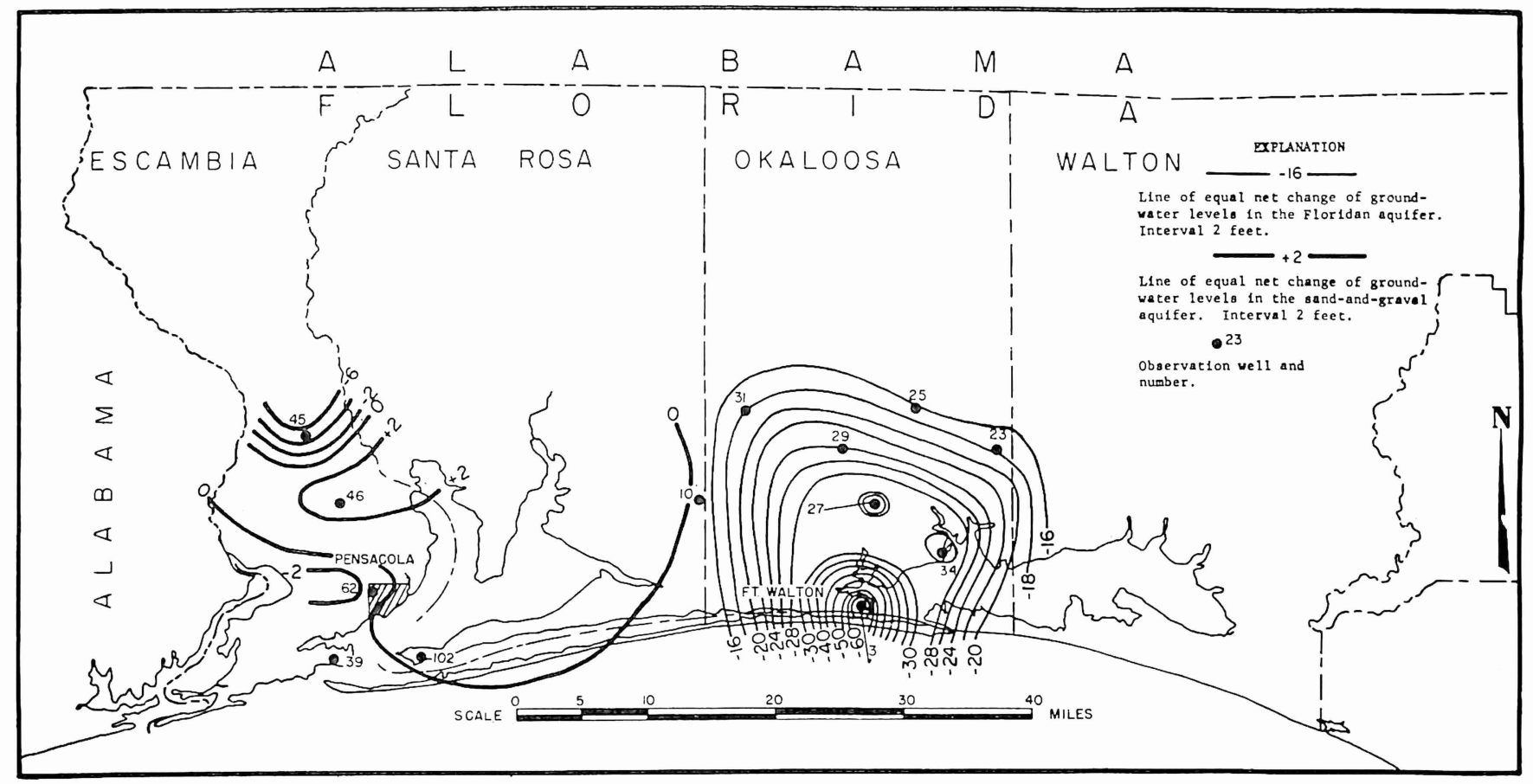

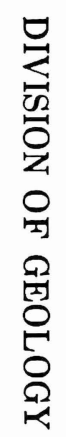

Figure 9. Map showing net change of ground-water levels, Pensacola and Ft. Walton areas, May 1951 to May 1962. 


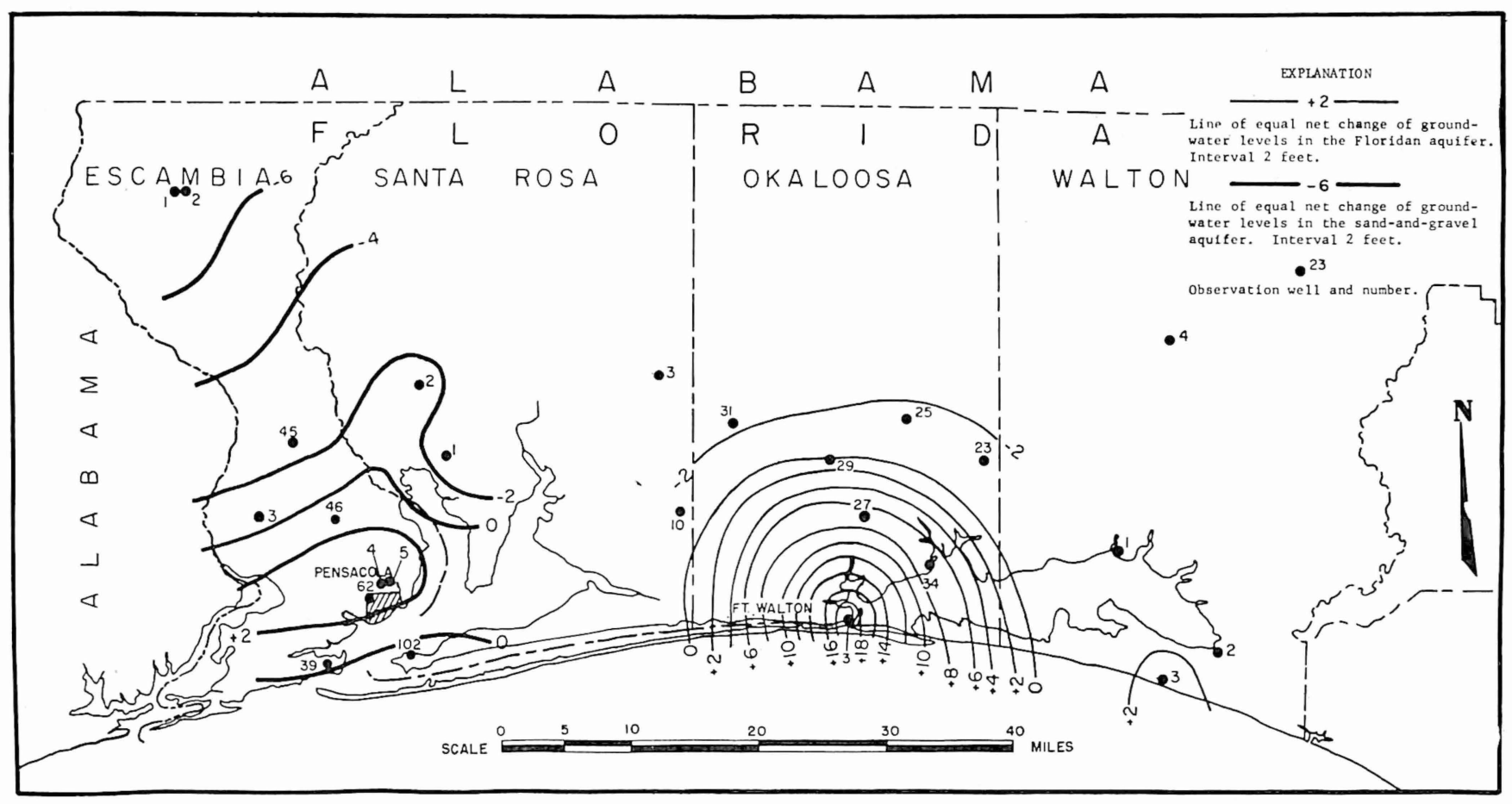

至

Figure 10. Map showing net change of ground-water levels, Pensacola and Ft. Walton areas, May 1962 to May 1964. 


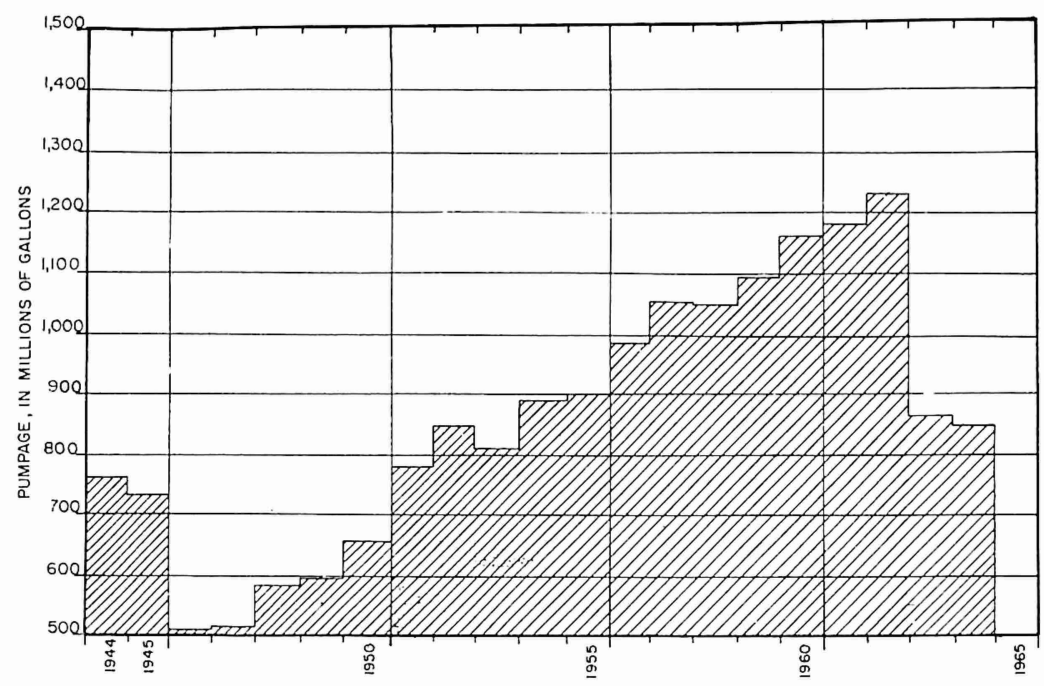

Figure 11. Graph of total yearly pumpage, Panama City.

central and eastern St. Johns, Flagler, and Volusia counties. Another source of water in these areas is a shallow, nonartesian, sand-and-shell aquifer.

\section{TALLAHASSEE AREA}

The Tallahassee area includes central Leon County and the City of Tallahassee. The area is primarily residential and only sparse light industry is located in this area.

The principal water user, the City of Tallahassee, supplies water for municipal use to the most rapidly growing residential and educational complex in the Big Bend. Since 1945, annual municipal pumpage at Tallahassee has increased 365 percent from about $850 \mathrm{mgy}$ to $3,100 \mathrm{mgy}$. Figure 14 shows pumpage during 1933-64 for the City of Tallahassee.

Fluctuations of water levels in the Floridan aquifer at Tallahassee are shown by the hydrograph, figure 15 , of well Leon 7 , which shows a downward trend during 1960-63 and an upward trend in response to above average rainfall during 1964. The graph shows characteristic seasonal trends with high levels in the spring and low levels in the fall. Figure 16 shows water levels in well Leon 7 for the period 1945-64. 

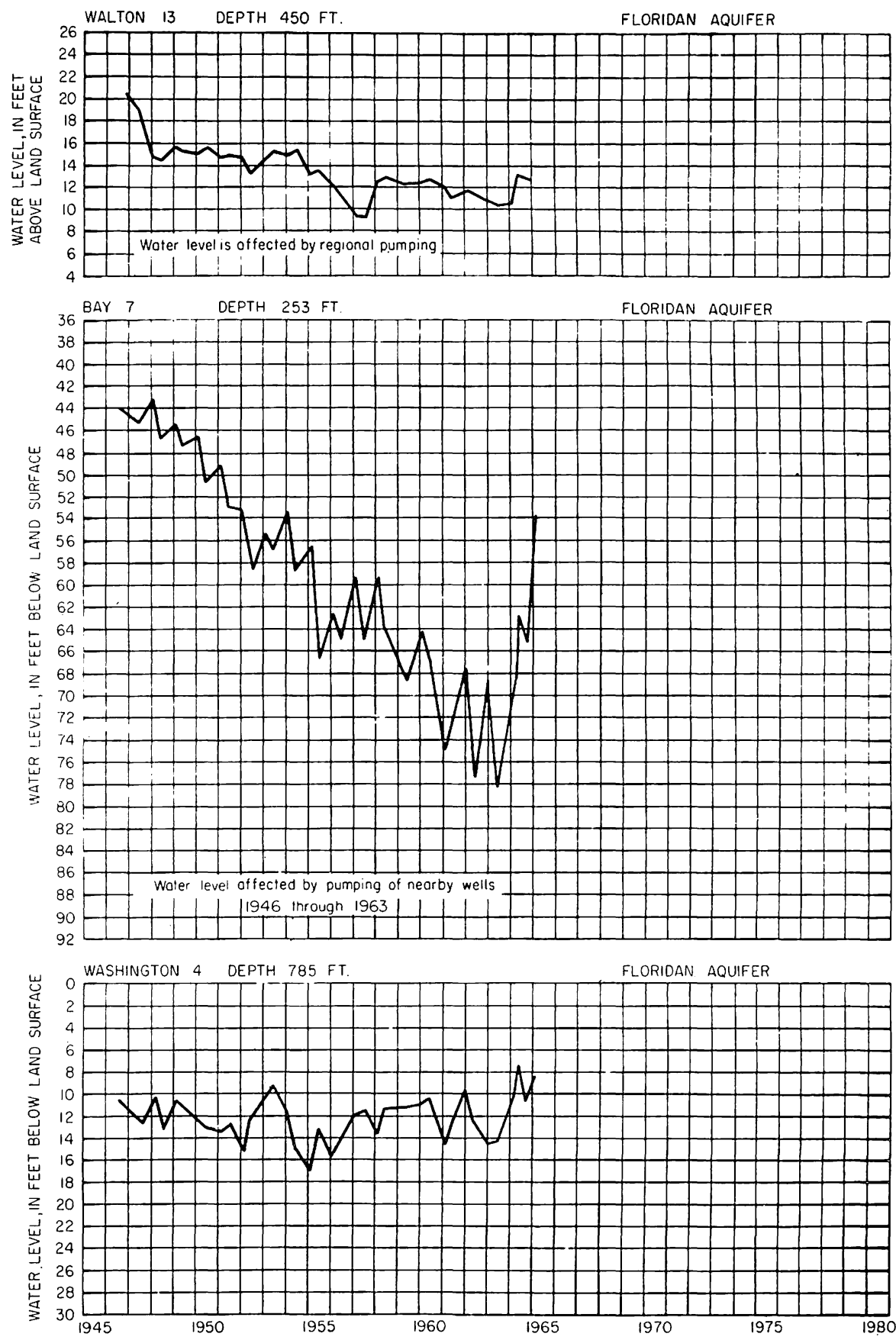

Figure 12. Hydrographs showing trends and fluctuations of water levels in wells Walton 13 at Point Washington, Bay 7 at Panama City, and Washington 4 , at Caryville. 


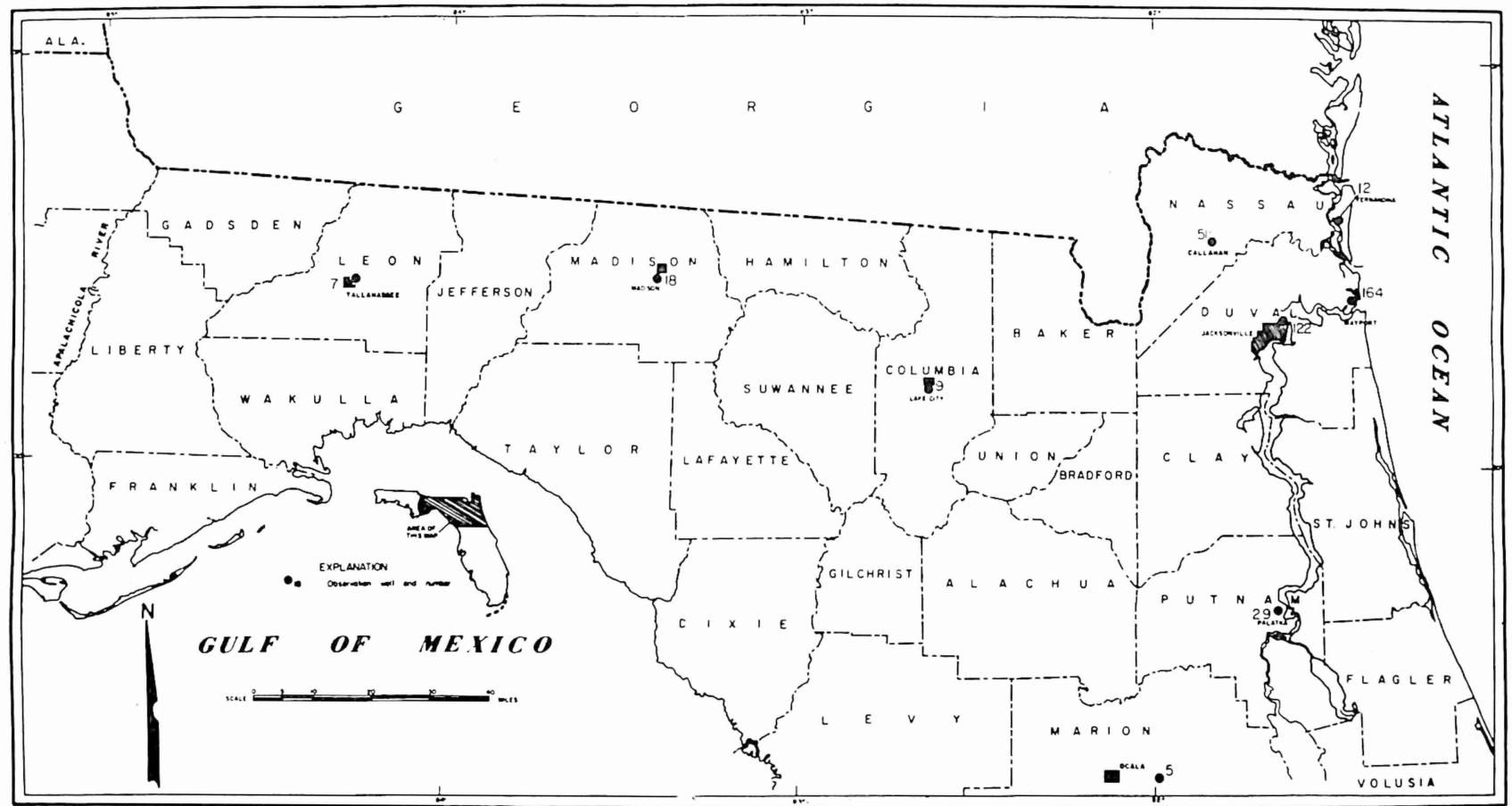

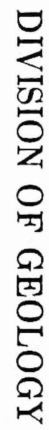

Figure 13. Map showing locations of observation wells in northern and north-central Florida for which hydrographs are given. 


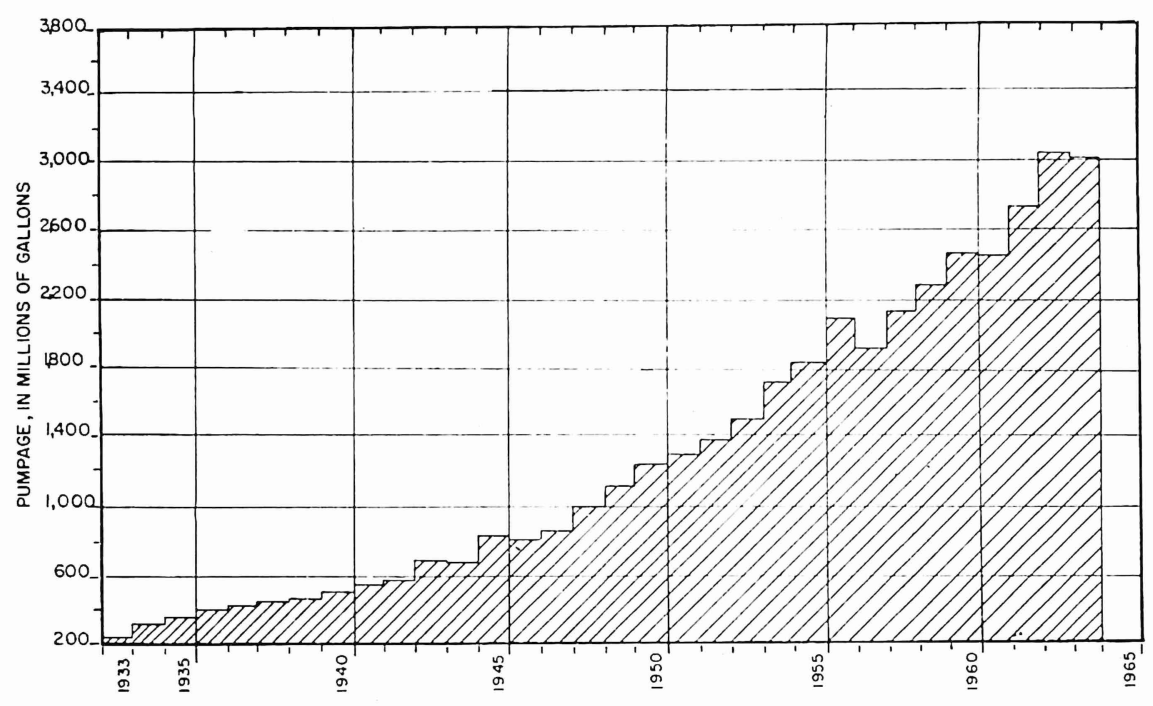

Figure 14. Graph of total yearly pumpage, City of Tallahassee.

FERNANDINA-JACKSONVILLE AREA

The Fernandina-Jacksonville area is one of the largest industrialized areas in the State, with water use increasing as a result of the rapid economic expansion. Figure 17 shows total yearly municipal pumpage for Jacksonville from 1921-64.

Ground-water levels in the Fernandina-Jacksonville area have been declining for a considerable period of time. Trends and seasonal fluctuations of the water levels in the Floridan aquifer at Jacksonville are shown for well Duval 122 and for well Duval 164 near Mayport. Hydrographs of wells in the Floridan aquifer in Nassau and Duval counties are shown in figures 16 and 18.

Water levels declined to near record low levels in many wells in the area during 1963. Maximum decline of water levels occurred in well Nassau 12 in the Fernandina area. Levels, in this well, declined 47 feet from 29 feet above to 18 feet below land surface during 1946-63. The maximum decline was 59 feet for the period of record 1939-63. In contrast, water levels in well Nassau 51, approximately 20 miles inland, declined only about 13 feet during 1945-46. Water levels in well Duval 122 at Jacksonville declined nearly 22 feet from 1930 to 1964, while along the coastal area in Duval County water levels generally declined about 18 feet in well Duval 164. These declines are part of the broad regional 

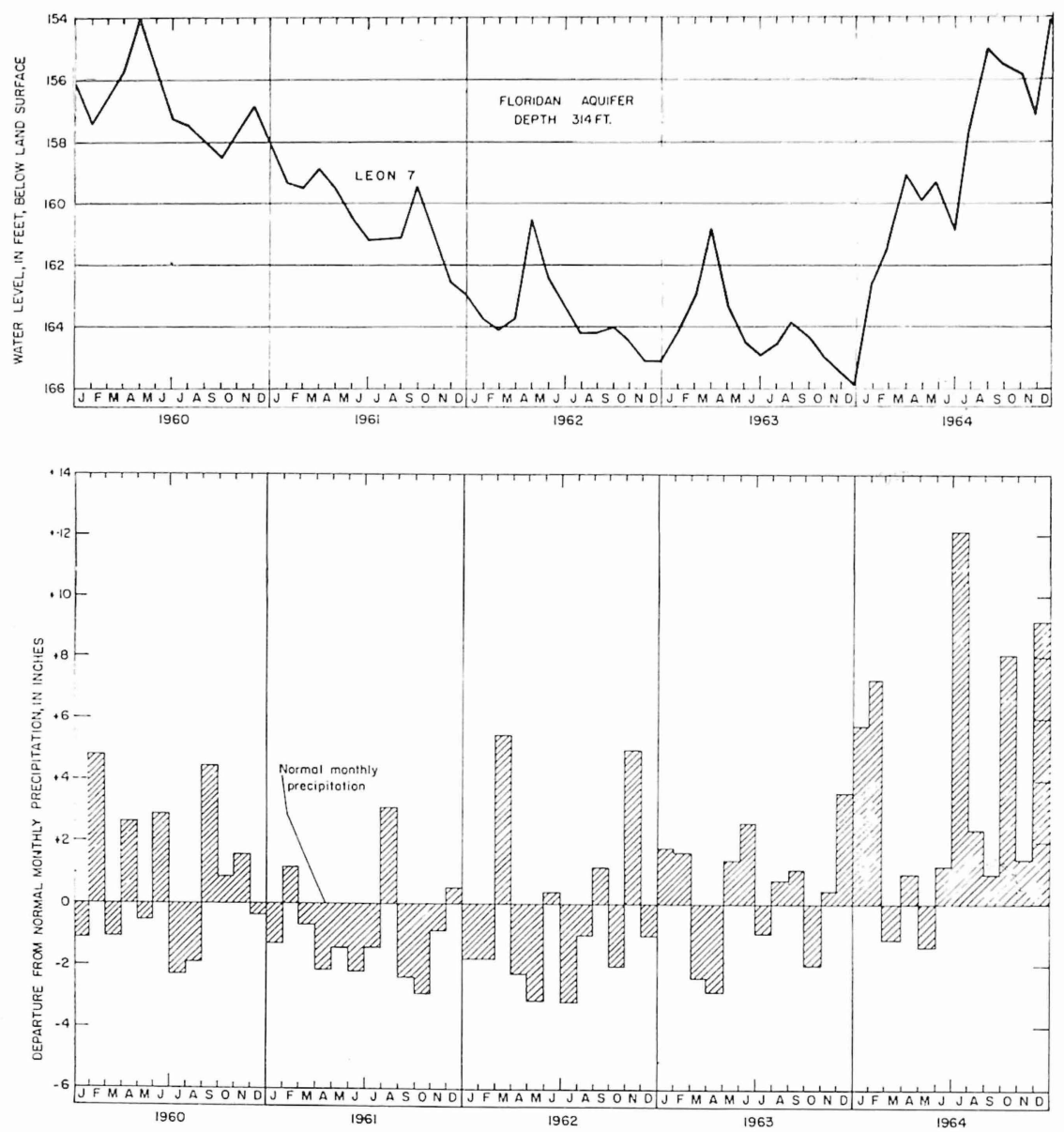

Figure 15. Hydrographs showing trends and fluctuations of water levels in well Leon 7 and departures from monthly normal precipitation at Tallahassee, 1960-64.

lowering of water levels as may be seen in figure 19, which shows the net change of ground-water levels in several counties.

\section{CENTRAL FLORIDA}

Central Florida, as used in this report, includes 20 counties and covers about 18,000 square miles. The extent of this region 

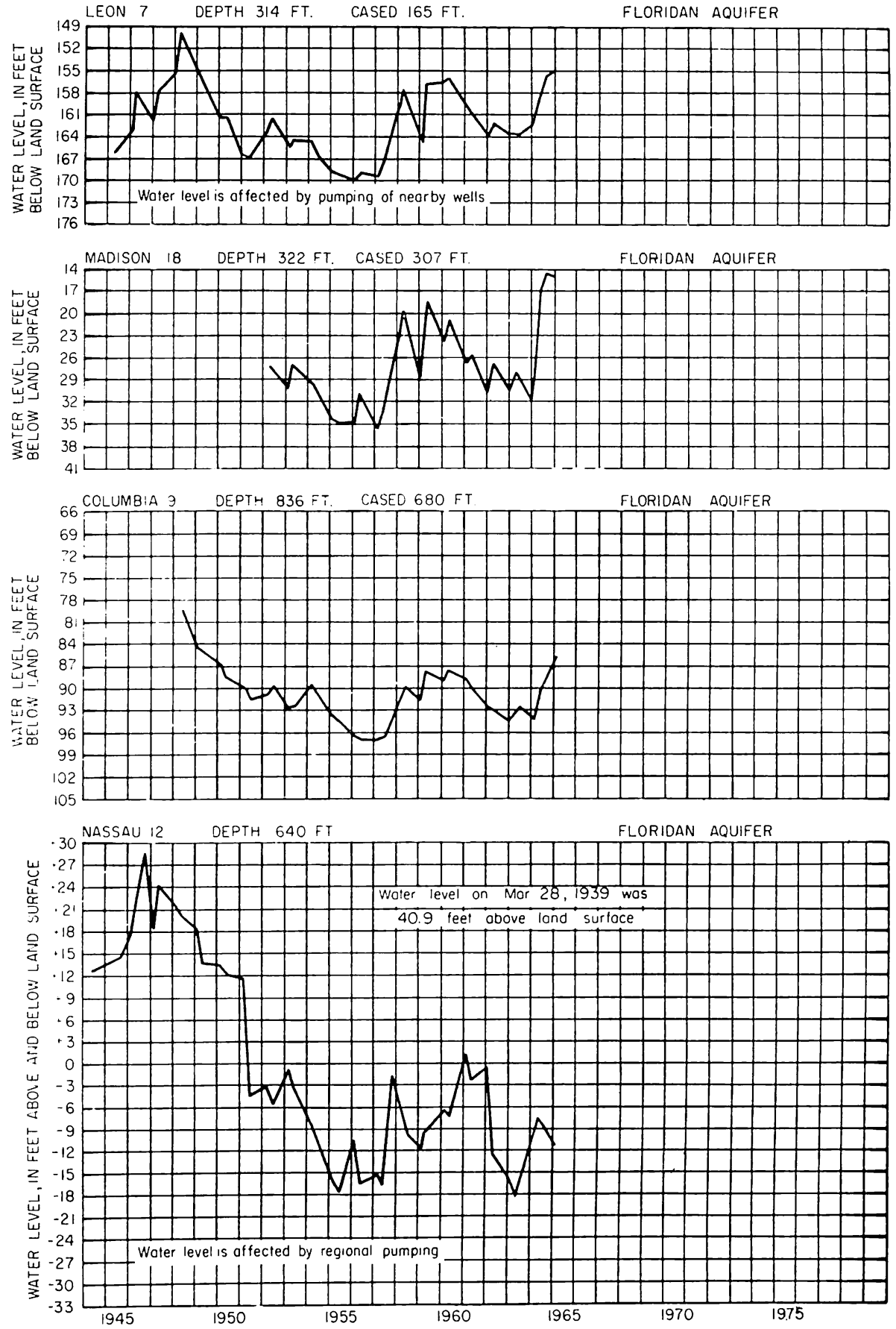

Figure 16. Hydrographs showing trends and fluctuations of water levels in wells Leon 7 at Tallahassee, Madison 18 near Madison, Columbia 9 at Lake City, and Nassau 12 near Fernandina. 


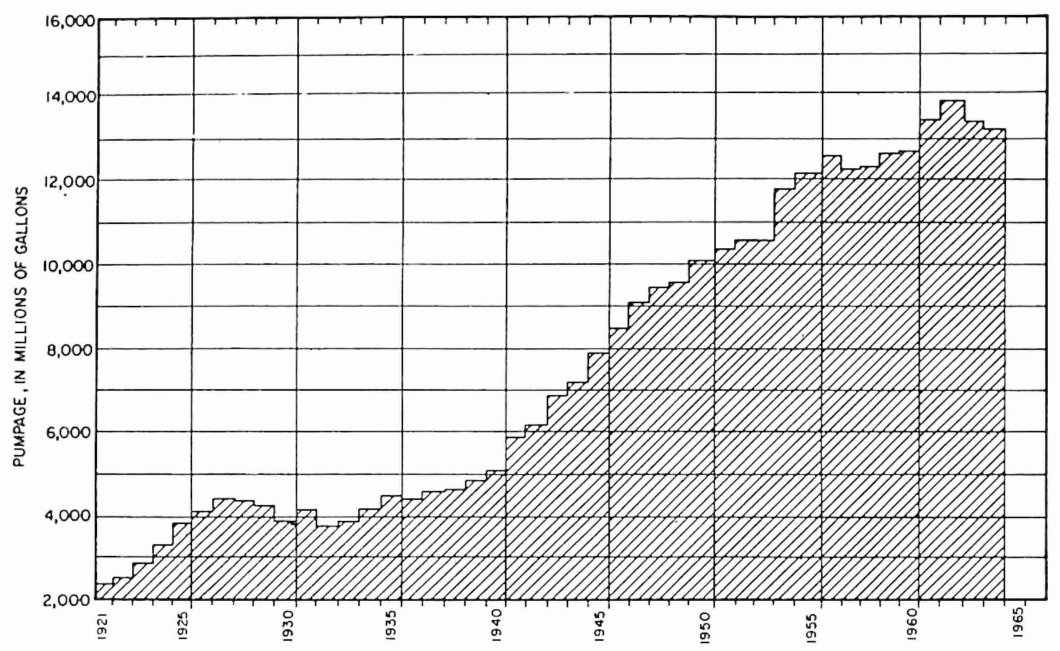

Figure 17. Graph of total yearly pumpage, City of Jacksonville.

and location of observation wells for which hydrographs are given are shown in figure 20.

The principal source of ground-water supply in western coastal and central peninsular Florida is the Floridan aquifer. In the eastern coastal area, the nonartesian shallow-sand aquifer is the chief source.

Central Florida includes four rapidly growing centers of population and industry: the Tampa-St. Petersburg area, the Lakeland area, the Orlando-Cape Kennedy area, the Sarasota-Bradenton area.

TAMPA-ST. PETERSBURG AREA

This area, particularly in the vicinity of Tampa and St. Petersburg, is undergoing a rapid expansion in both industry and population.

The long-term trends and fluctuations of ground-water levels in the Floridan aquifer in the Tampa-St. Petersburg area are shown in figure 21. The hydrographs show a similarity of waterlevel fluctuations in well Pasco 13 near Ehren and well Hillsborough 13 near Citrus Park, during the period 1945 through 1962. Drought conditions and increased pumping during 1961-64 caused water levels in Hillsborough 13 to decline to the lowest levels of record in 1964. Rainfall recorded at Tampa and the fluctuations of the water level in well Hillsborough 13 for the period 1960-64 

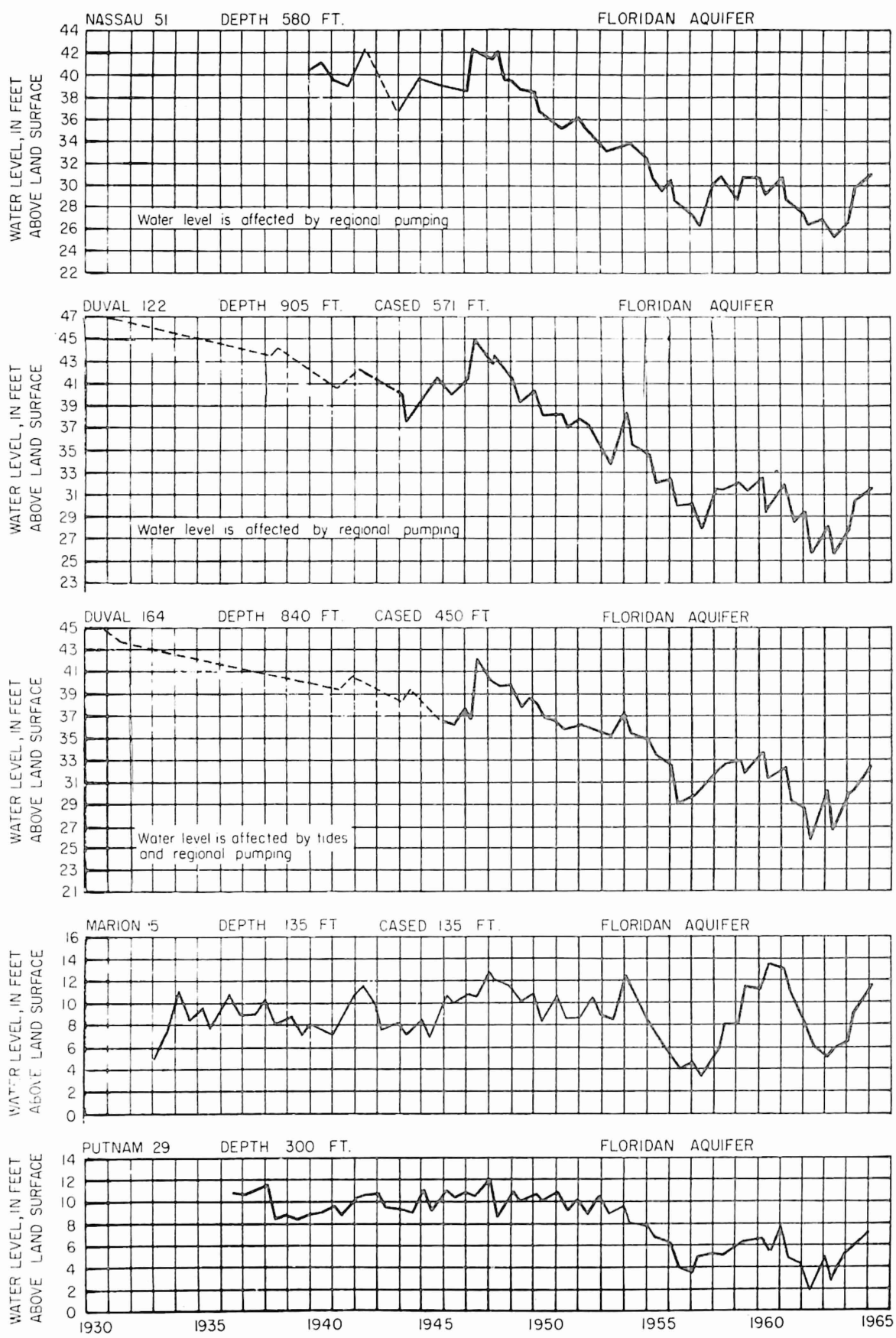

Figure 18. Hydrographs showing trends and fluctuations of water levels in wells Nassau 51 at Callahan, Duval 122 at Jacksonville, Duval 164 near Mayport, Marion 5 near Ocala, and Putnam 29 at Palatka. 

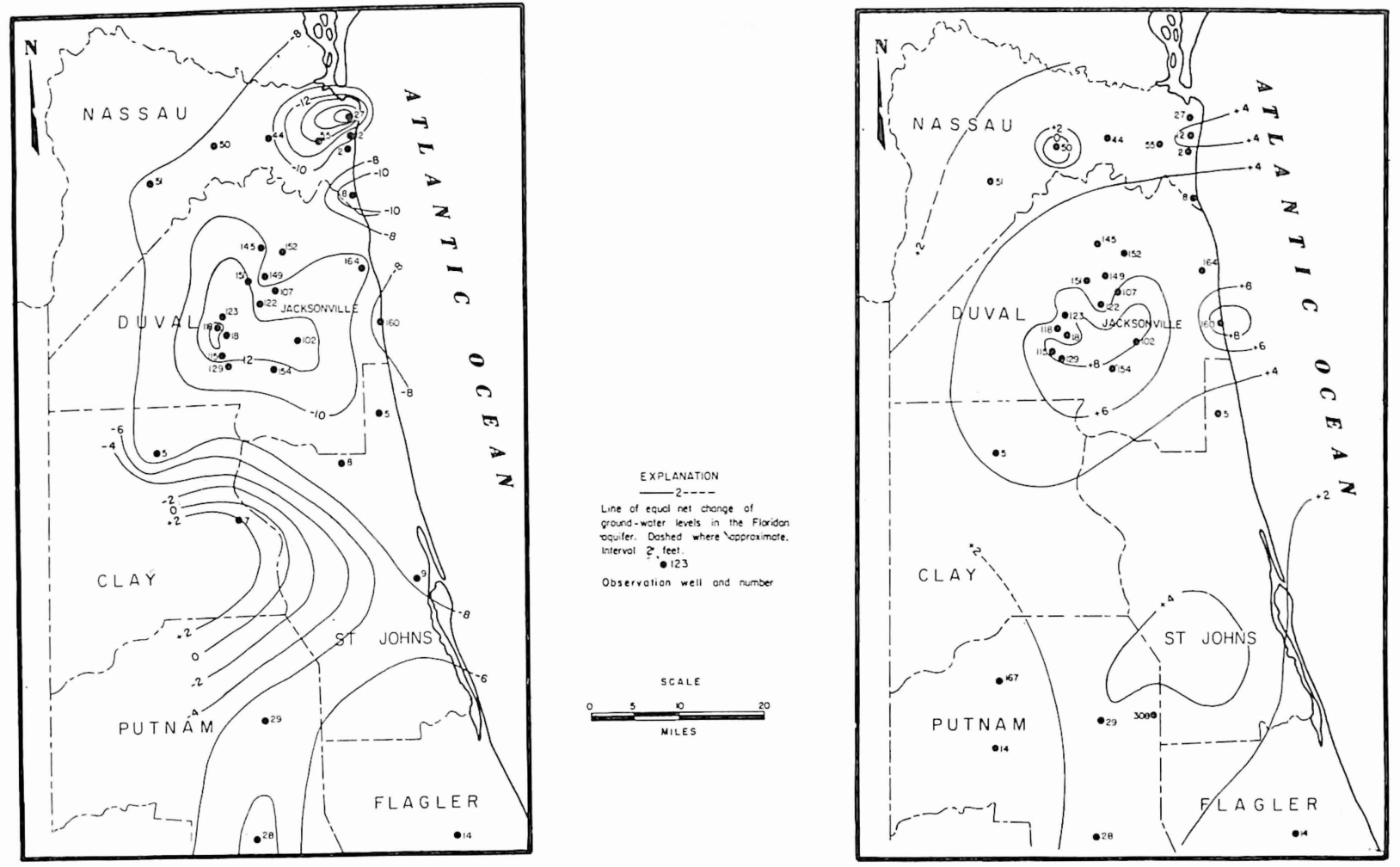

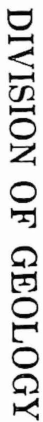

Figure 19. Maps showing net changes of ground-water levels in Jacksonville and Fernandina areas, May 1951 to May 1962 and from May 1962 to May 1964. 


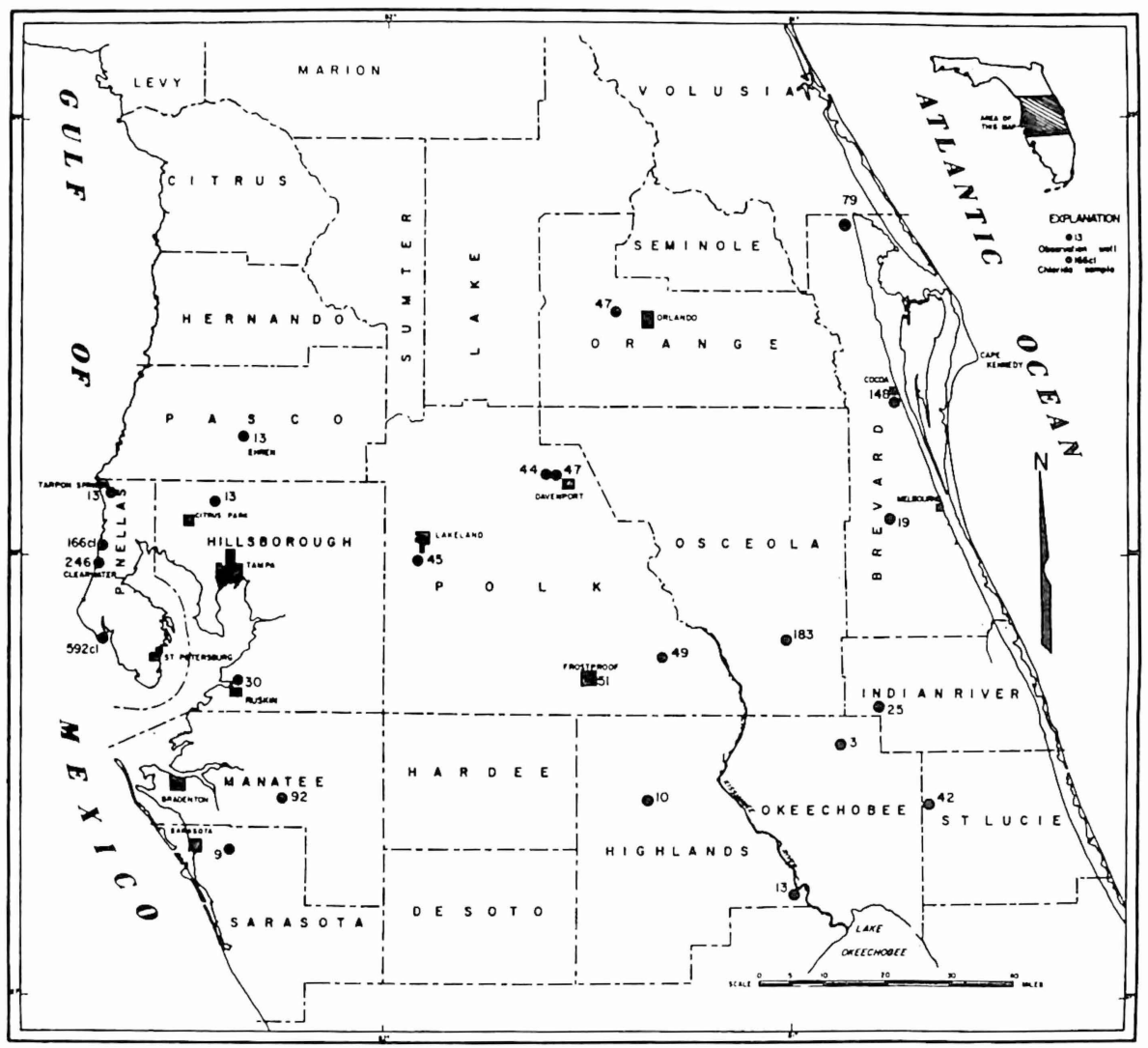

Figure 20. Map showing locations of observation wells in central Florida for which hydrographs are given.

is shown in figure 22. Near Ruskin, in southern Hillsborough County, water levels in well Hillsborough 30 declined to the lowest level of record in 1963, shown in figure 23. This decline is part of an extensive regional lowering of water levels which extends from southern Hillsborough County into Sarasota County. (See figure on page 54).

Water levels in two Pinellas County wells, Pinellas 13 and 246 , are shown in figure 23. No apparent trend is noted for Pinellas 13. However, a slight downward trend from 1946 through 1956 can be noted for Pinellas 246. This downward trend was reversed during the latter part of 1956 and levels continued to rise through 1959 and were about average for the period of record 1963-64.

The changes in chloride content of water from two wells in the Floridan aquifer in Pinellas County are shown in figure 24. 

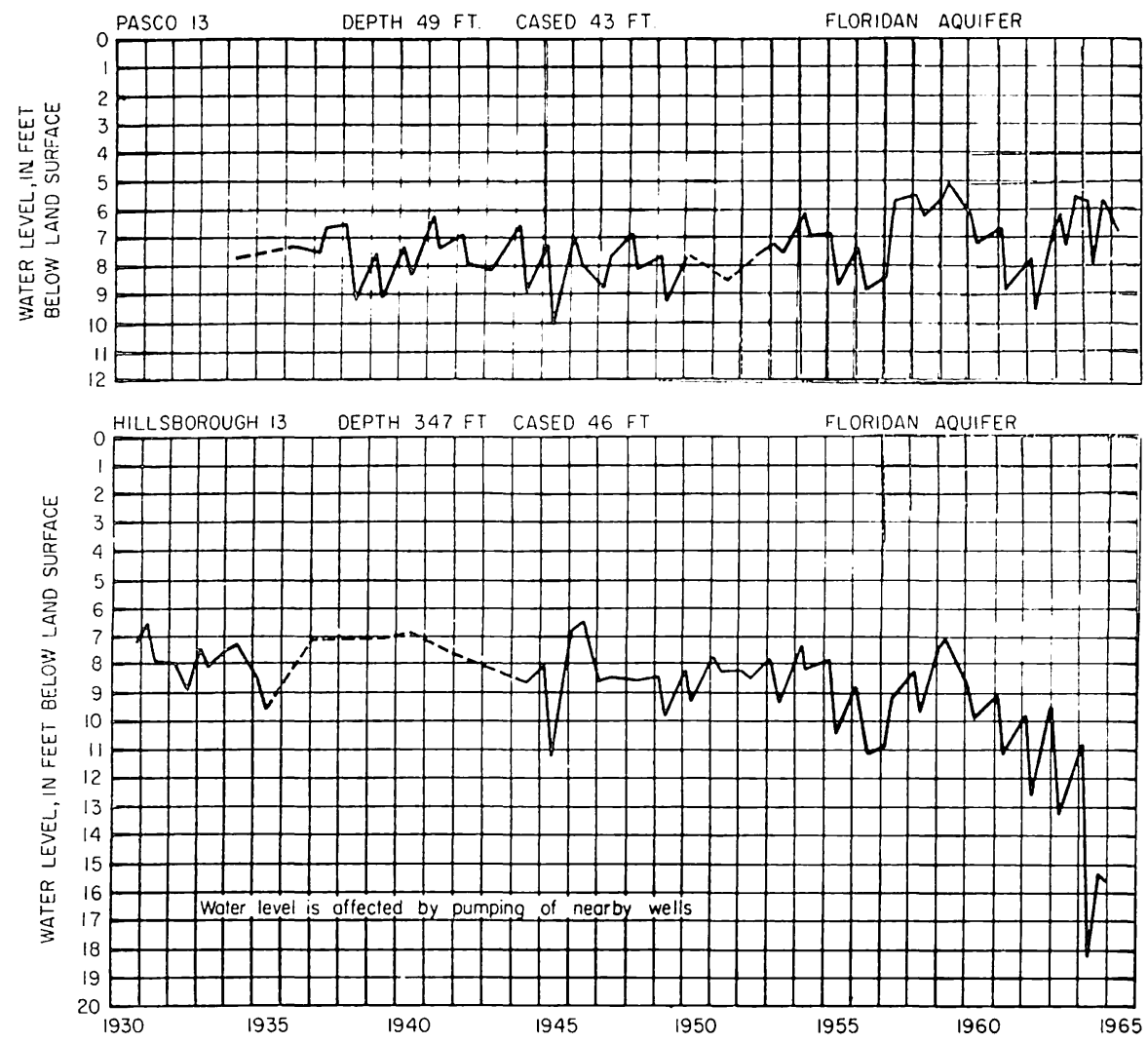

Figure 21. Hydrographs showing trends and fluctuations of water levels in wells Pasco 13 near Ehren and Hillsborough 13 near Citrus Park, Tampa area.

The chloride content of well Pinellas 592 at Bay Pines ranged from $1,000 \mathrm{ppm}$ (parts per million) to 2,200 ppm from 157-64. The chloride content of well Pinellas 166 at Dunedin ranged from about $20 \mathrm{ppm}$ to $1,000 \mathrm{ppm}$ during the same period. The chloride content in both wells decreased during 1957-61. During 1963-64 the chloride content in well 592 increased nearly to the 1959 concentration. In contrast, the chloride content in well 166 remained low during 1963-64 and at the end of the year was well below the 1959 concentration.

\section{LAKELAND AREA}

In the Lakeland area, like others in Florida, ground water is being pumped at an increasing rate commensurate with the econo- 

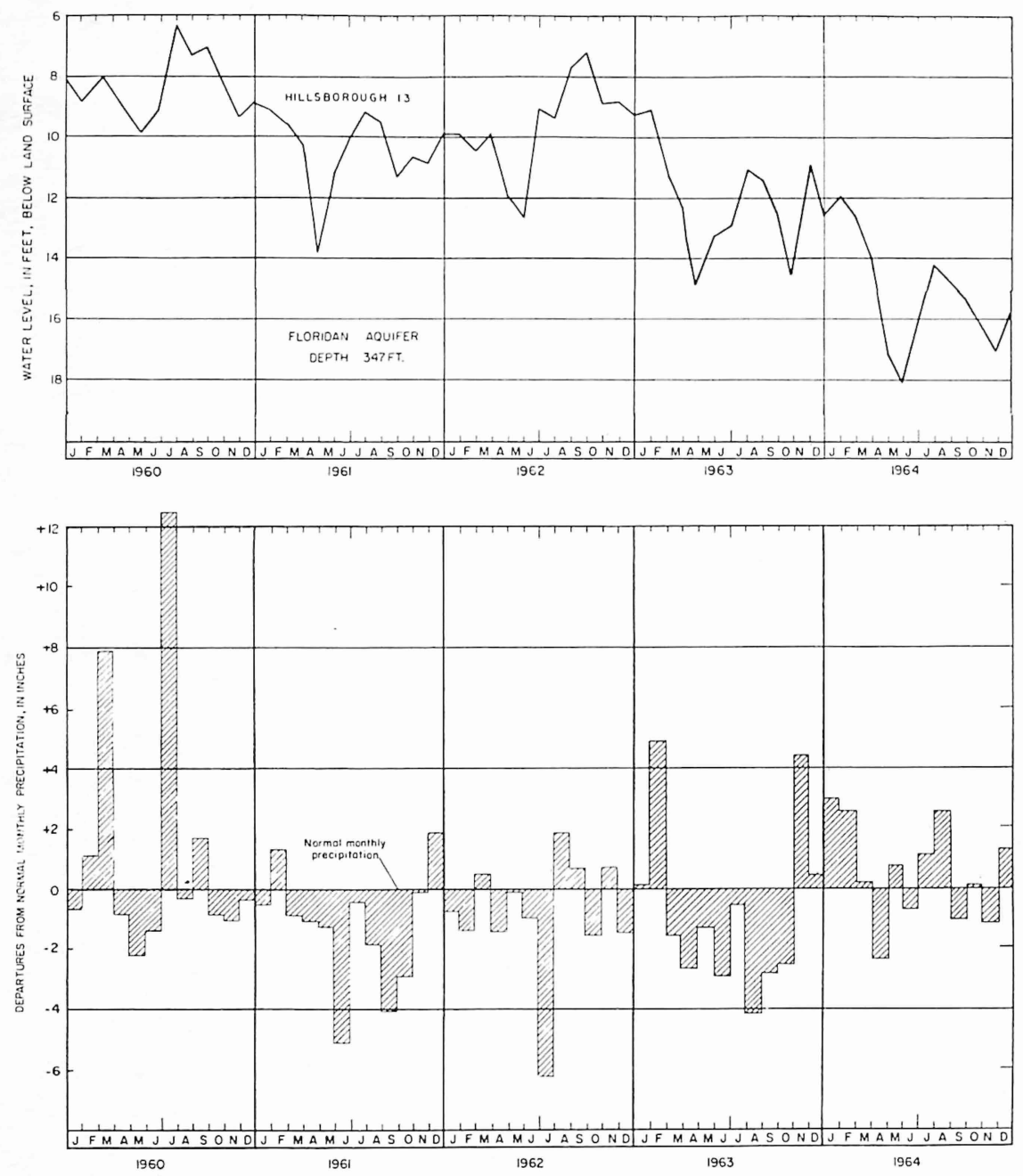

Figure 22. Hydrographs showing trends and fluctuations of water levels in well Hillsborough 13 and departures from monthly normal precipitation at Tampa, 1960-64.

mic growth of the area. Municipal pumpage at Lakeland increased about 118 percent during the 12-year period 1953-64. Figure 25 shows the total yearly municipal pumpage at Lakeland for 1928-64. Annual industrial pumpage in Polk County is presently (1964) about 68,000 million gallons.

The marked decline of water levels in the Floridan aquifer in the vicinity of Lakeland is shown in figure 26. A maximum decline about 9 feet in well Polk 45 occurred during May 1960 through May 1962. 

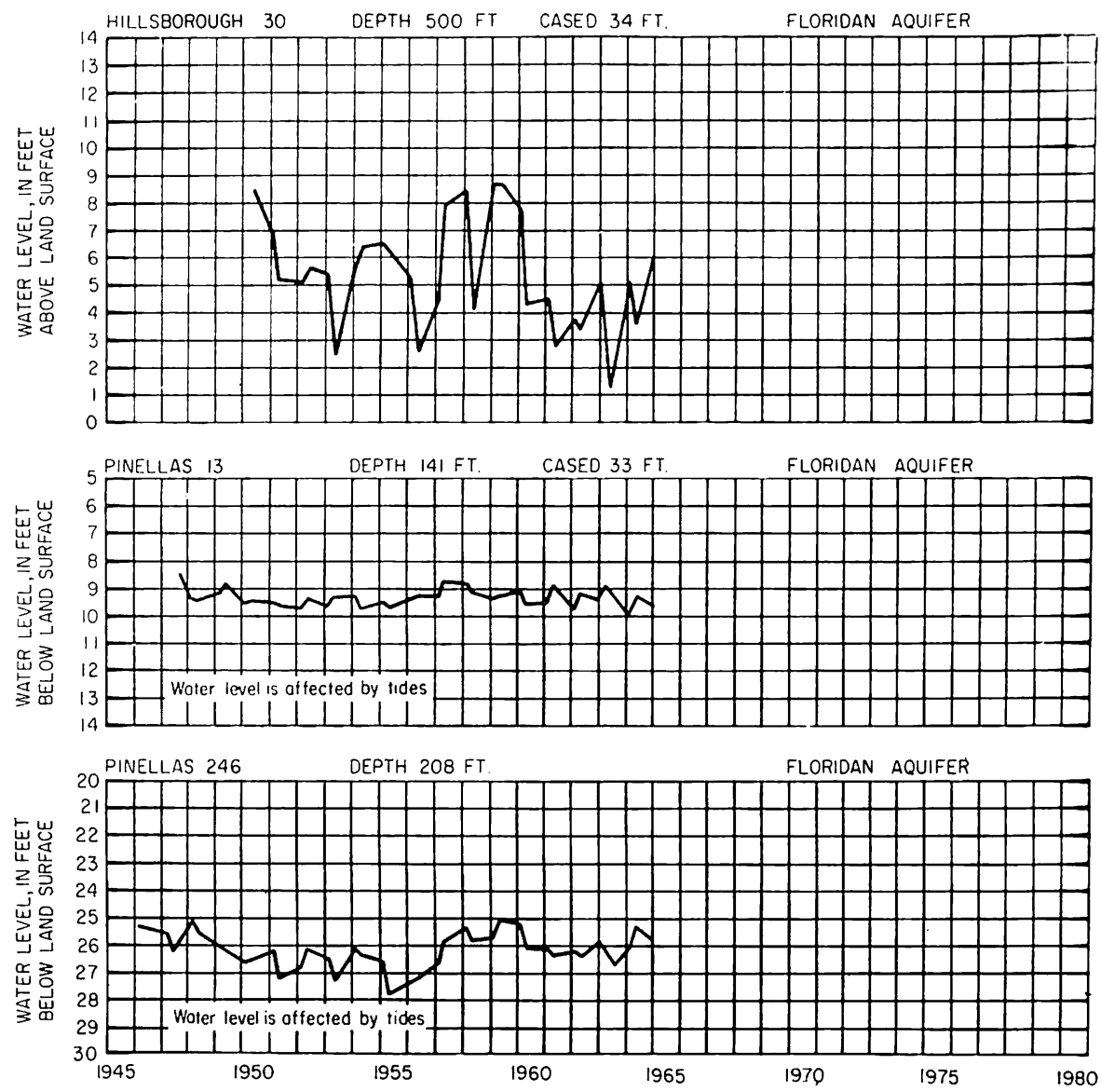

Figure 23. Hydrographs showing trends and fluctuations of water levels in wells Hillsborough 30 near Ruskin, Pinellas 13 at Tarpon Springs, and Pinellas 246 at Clearwater.

Precipitation recorded at Lakelard shows deficient rainfall in the Lakeland area during the years 1961 through 1964.

In many areas in northern Polk County, water levels after declining to new record low levels during 1960-62 rose sharply in 1963 and declined slightly in 1964. From record low water levels of May 1962, levels in the Floridan aquifer recovered nearly 12 feet in well Polk 45 in the heavily pumped area south of Lakeland and nearly 3 feet in well Polk 44 near Davenport in northeastern Polk County. Levels in the nonartesian aquifer in well Polk 47 near Davenport rose about 2 feet in 1963. Although water levels rose during 1963, downward trends of levels in artesian and nonartesian aquifers continued. During 1964 water levels ranged from 


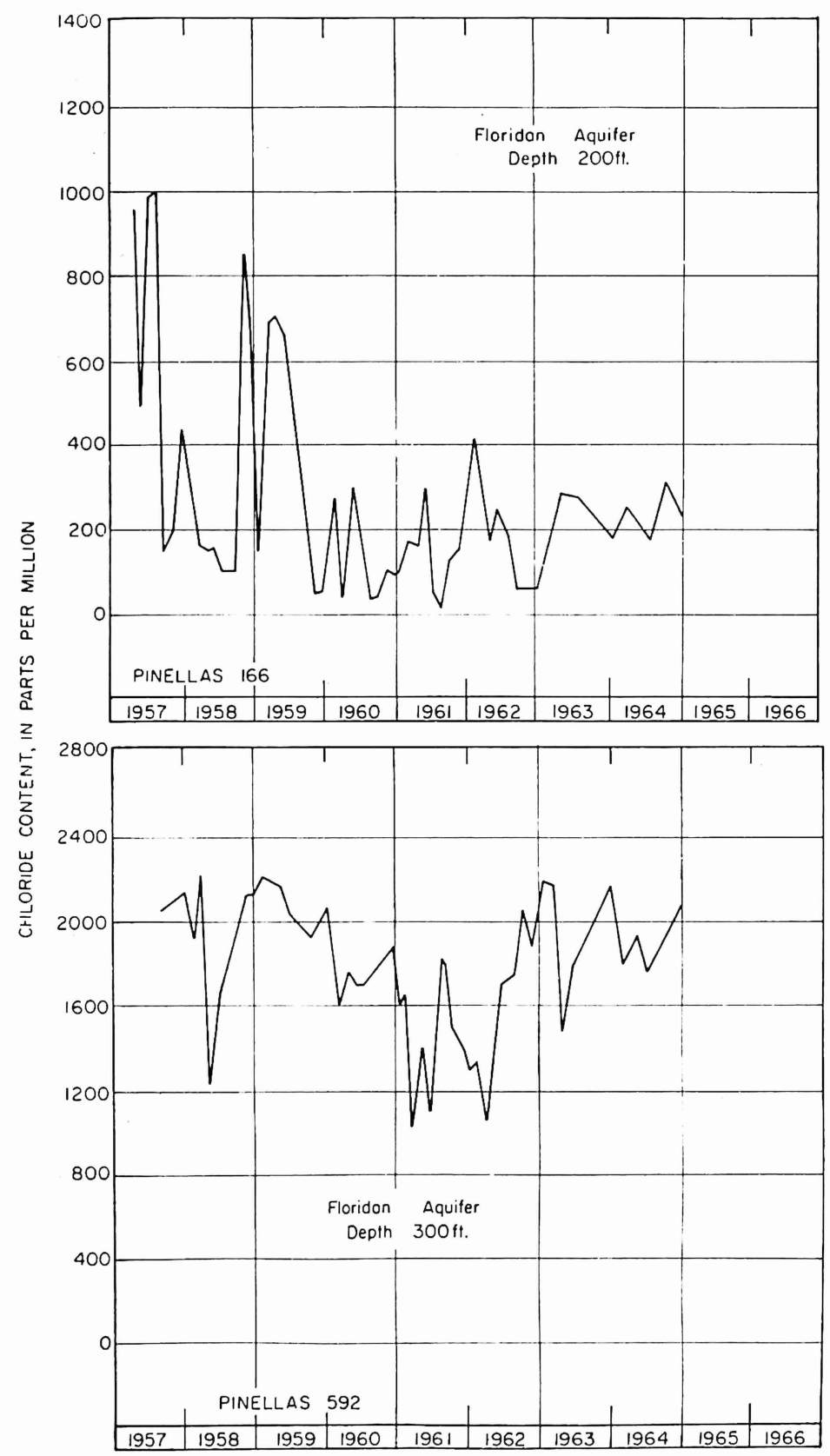

Figure 24. Graphs showing changes in chloride content in wells Pinellas 592 at Bay Pines and 166 at Dunedin, St. Petersburg area. 


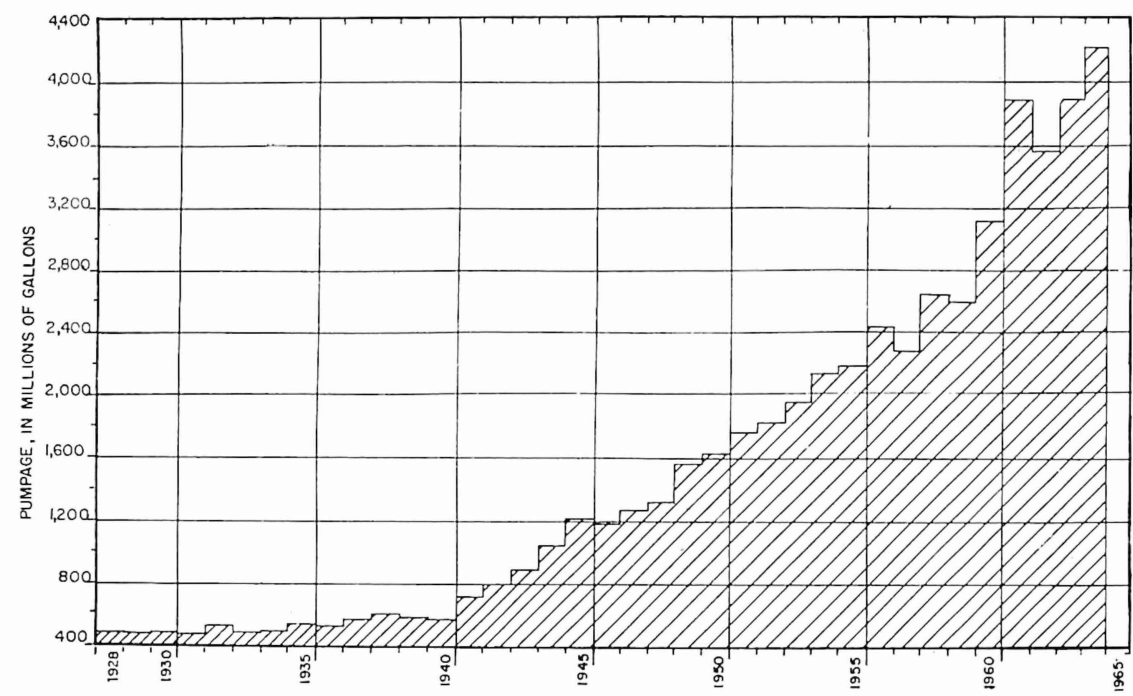

Figure 25. Graph showing total yearly pumpage, City of Lakeland.

8.5 feet below 1960 highest levels in the Floridan aquifer near Lakeland to 1 foot lower than 1960 levels in the shallow-sand nonartesian aquifer near Davenport. Long-term trends and fluctuations of ground-water levels in the Lakeland area are shown in figure 27.

Water levels in the artesian aquifer, in southern Polk County, and in the shallow-sand aquifer, in southeastern Polk County and in central Highlands County, are shown in figure 28.

The most prominent features illustrated by the hydrographs in figure 28 are the fluctuation of water levels in the artesian aquifer and in the shallow-sand aquifer caused by the droughts of 1955-56 and 1961-62 and subsequent recovery of levels during post-drought periods.

In southern Polk County at Frostproof, water levels declined about 11 feet in the artesian aquifer in well Polk 51 from January 1960 to May 1962. In central Highlands County near Sebring, levels declined nearly 6 feet in the nonartesian aquifer in well Highlands 10 during the same period. During 1962 ground-water levels rose sharply. However, in most wells, the recovery of levels in 1963-64 did not exceed those of 1960 . Water levels during 1964 ranged from 1.5 feet below 1960 highest levels in the artesian aquifer at Frostproof to 5.1 feet lower than 1960 levels in the nonartesian aquifer in central Highlands County. In southern Osceola and southeastern Highlands counties, 1963-64 water levels in the nonartesian aquifer ranged from 1 foot lower in wells Osceola 183 and Okeechobee 3 to 2.4 feet lower than 1960 levels in well Highlands 13 . Figure 29 

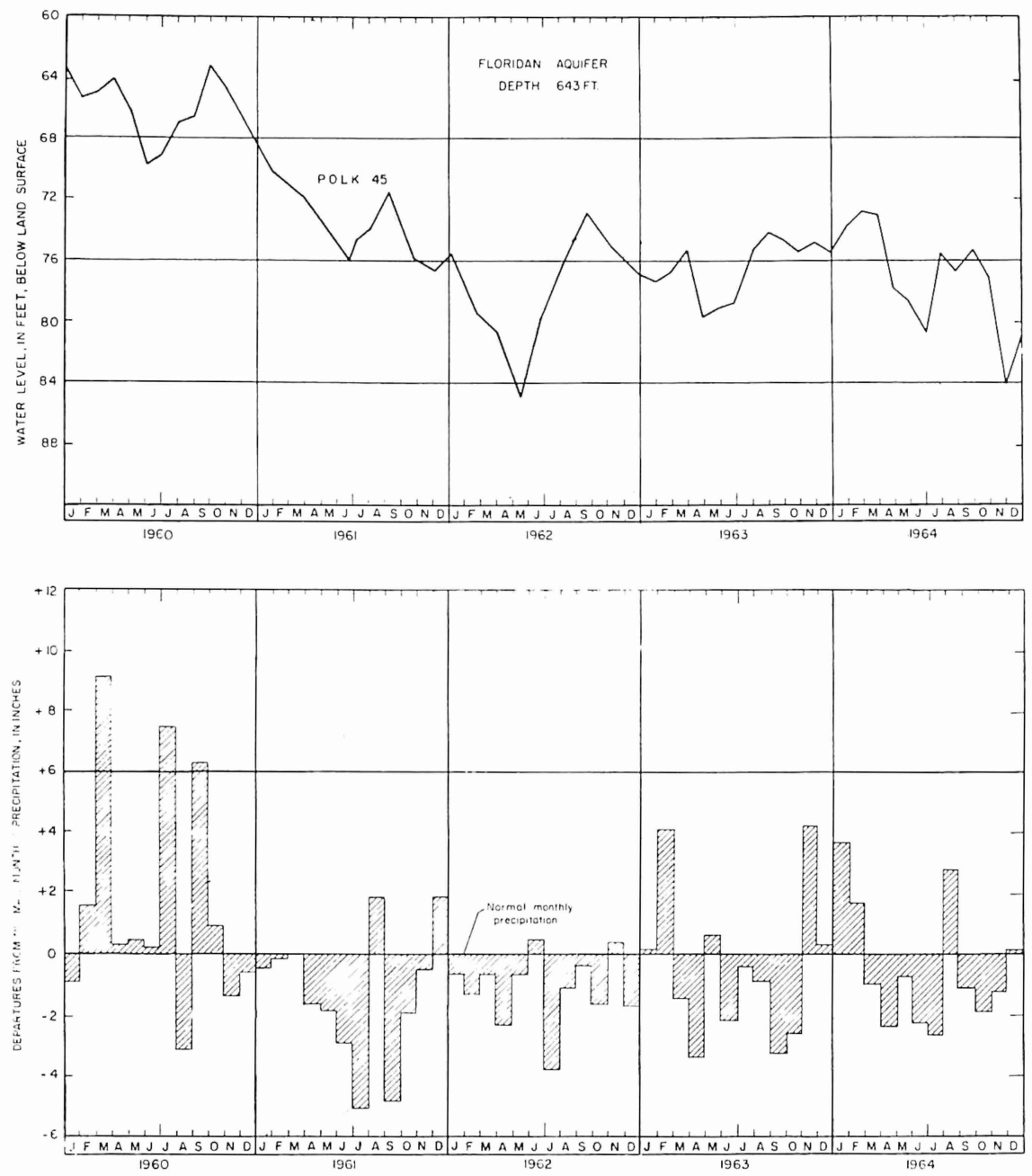

Figure 26. Hydrographs showing trends and fluctuations of water levels in well Polk 45 near Lakeland and departures from monthly normal precipitation at Lakeland, 1960-64.

shows fluctuations of water levels in the shallow nonartesian aquifer in Highlands, Okeechobee, and Osceola counties.

\section{ORLANDO AREA}

The Orlando area includes the cities of Orlando, Winter Park, and Maitland in north central Orange County. The Floridan aquifer supplies most of the water for municipal and industrial needs in the area. Trends and fluctuations of ground-water levels in the 

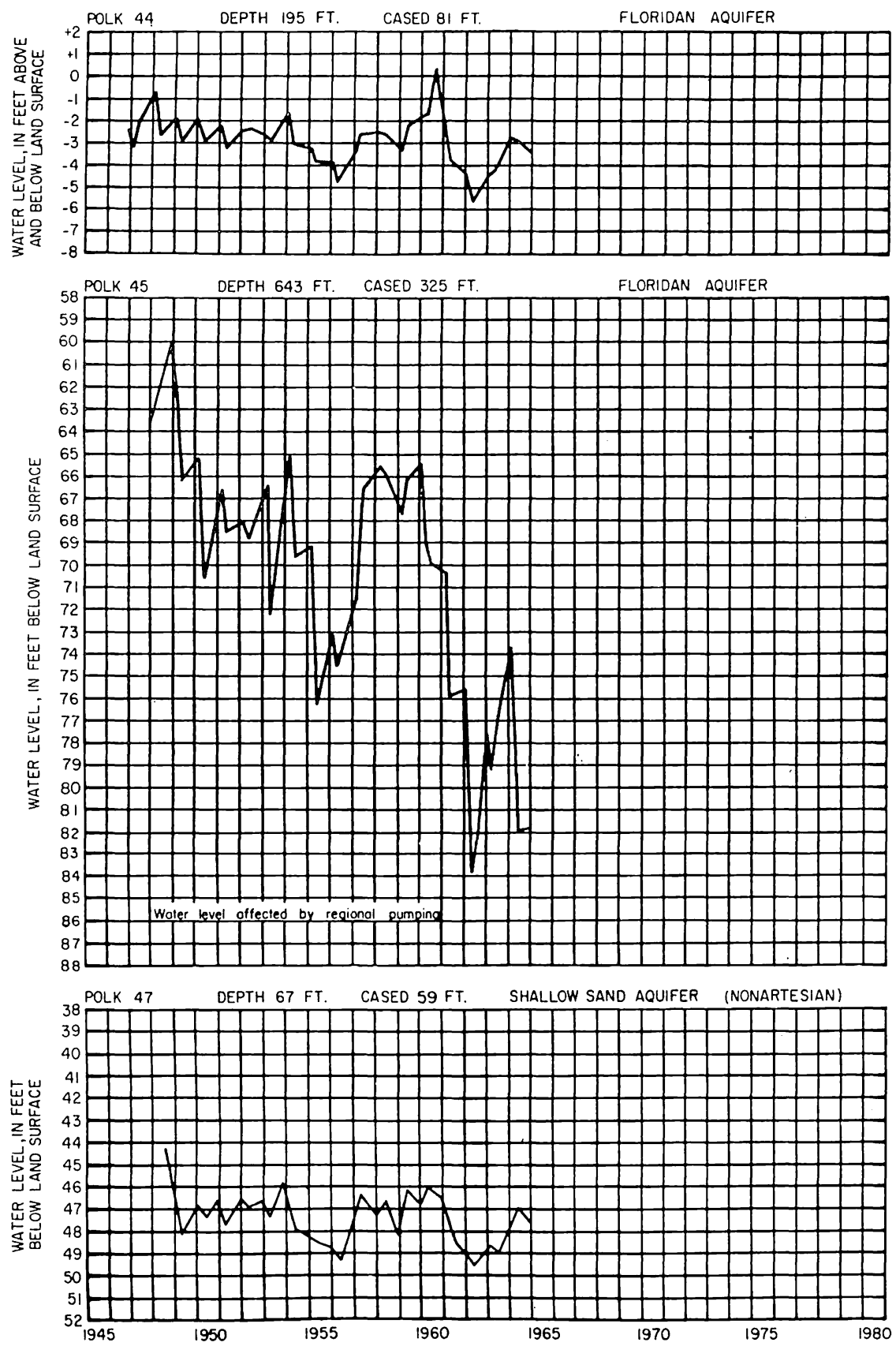

Figure 27. Hydrographs showing trends and fluctuations of water levels in wells Polk 44 and 47 near Davenport and Polk 45 near Lakeland, Lakeland area. 

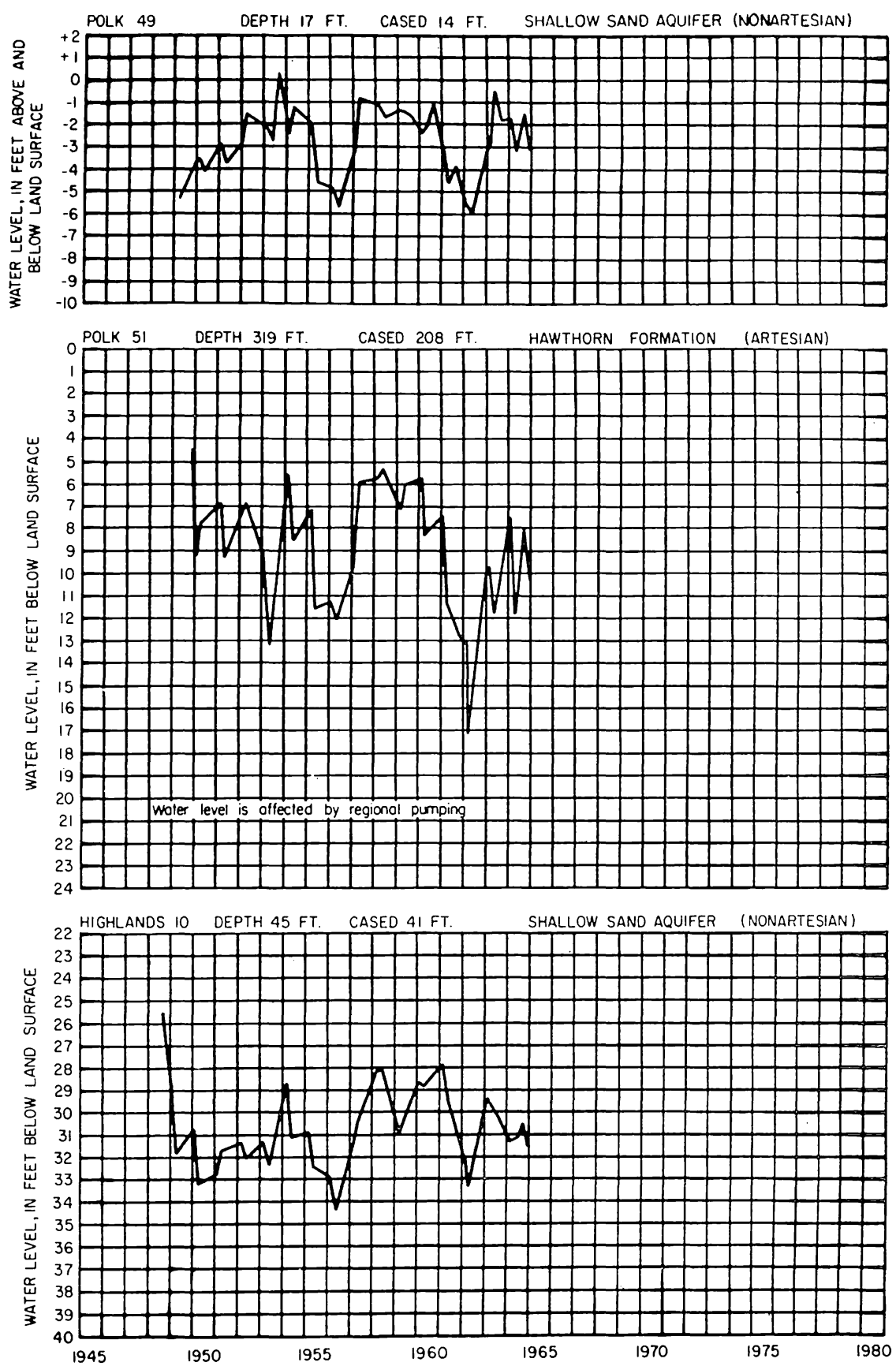

Figure 28. Hydrographs showing trends and fluctuations of water levels in wells Polk 49 near Frostproof and Polk 51 at Frostproof, and Highlands 10 near Sebring. 

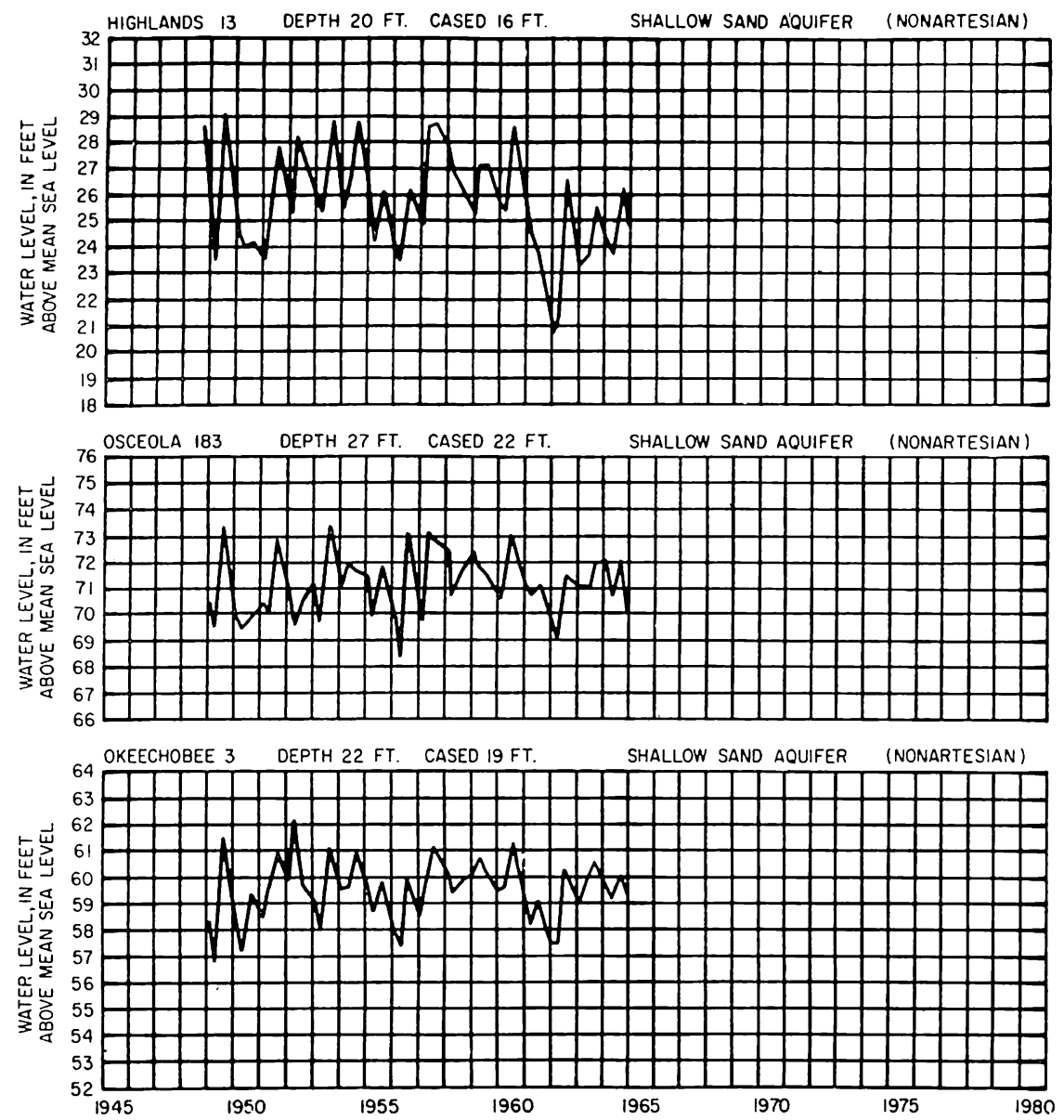

Figure 29. Hydrographs showing trends and fluctuations of water levels in wells Highlands 13, Osceola 183, and Okeechobee 3 in the Kissimmee Valley.

Floridan aquifer and nonartesian aquifer near Orlando are shown in figure 30 . The long-term trend of artesian water levels in the Floridan aquifer in the Orlando area is illustrated in figure 31. The hydrograph of well Orange 47 shows levels declined from the highest of record in the spring of 1960 to a new low of record in 1962. A maximum fluctuation of 22 feet was recorded during this period. From May 1962 to September 1964 levels rose about 8 feet, however, they remained below the average level of previous years. 

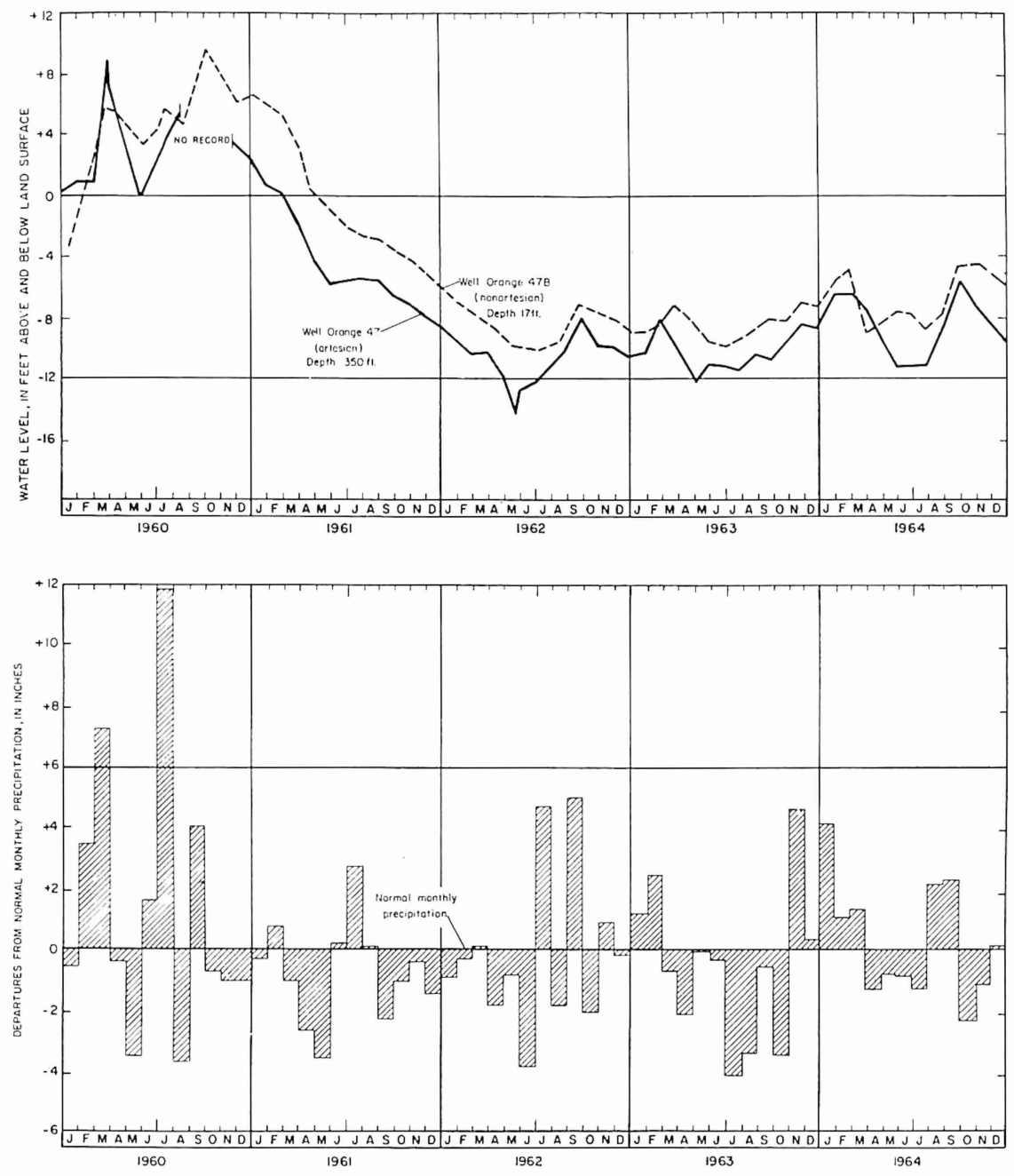

Figure 30. Hydrographs showing trends and fluctuations of water levels in wells Orange 47 and $47 \mathrm{~B}$ near Orlando and departures from monthly normal precipitation at Orlando, 1960-64.

\section{CAPE KENNEDY AREA}

The Cape Kennedy area, one of the most rapidly growing areas in the State, includes the cities of Cocoa, Cocoa Beach, and Titusville in Brevard County. Water in the Floridan aquifer in the area is generally brackish and is used primarily for irrigation. Figure 32 shows water-level fluctuations in eastern coastal Florida in Brevard, Indian River, and St. Lucie counties.

Hydrographs of wells in Brevard County generally show a 


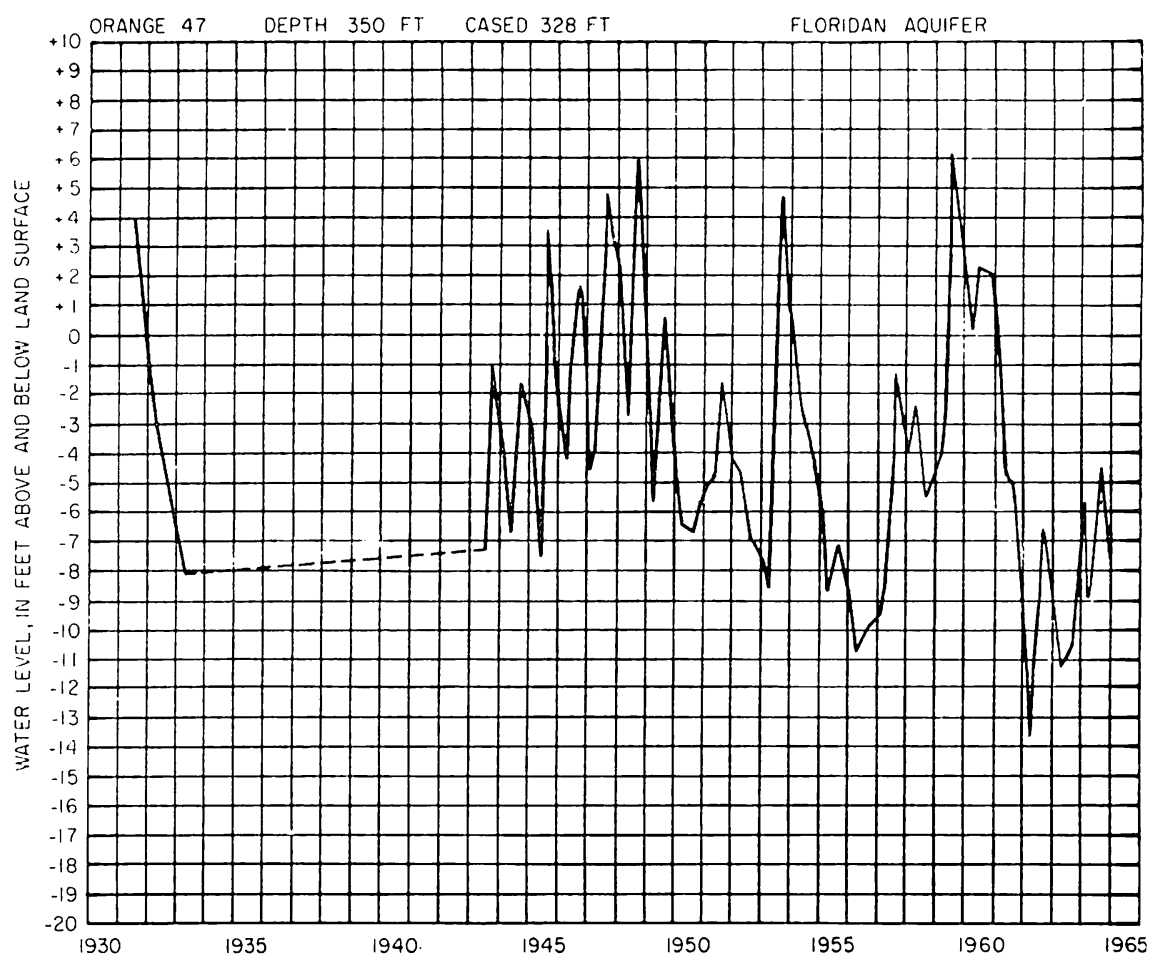

Figure 31. Hydrographs showing trends and fluctuations of water levels in well Orange 47, near Orlando.

long-term downward average trend of artesian levels in the Floridan aquifer. Since 1946, artesian water levels have declined about 6 feet in well Brevard 19 near Melbourne and Eau Gallie and about 8 feet in well Brevard 148 at Cocoa. Levels have declined about 4 feet in well Brevard 79 in northern Brevard County about 28 miles northwest of Cape Kennedy.

Hydrographs of wells in the shallow-sand aquifer in Indian River and St. Lucie counties indicate no apparent downward trend of ground-water levels during the period of record.

\section{SARASOTA-BRADENTON AREA}

The Sarasota-Bradenton area includes Manatee and Sarasota counties in southwestern coastal Florida. The principal economic activities in the area are agriculture and stock raising. The coastal section, however, is rapidly developing as a retirement and yearround tourist center.

Figure 33 shows the trends and fluctuations of water levels in observation wells Manatee 92 and Sarasota 9. Hydrographs of 

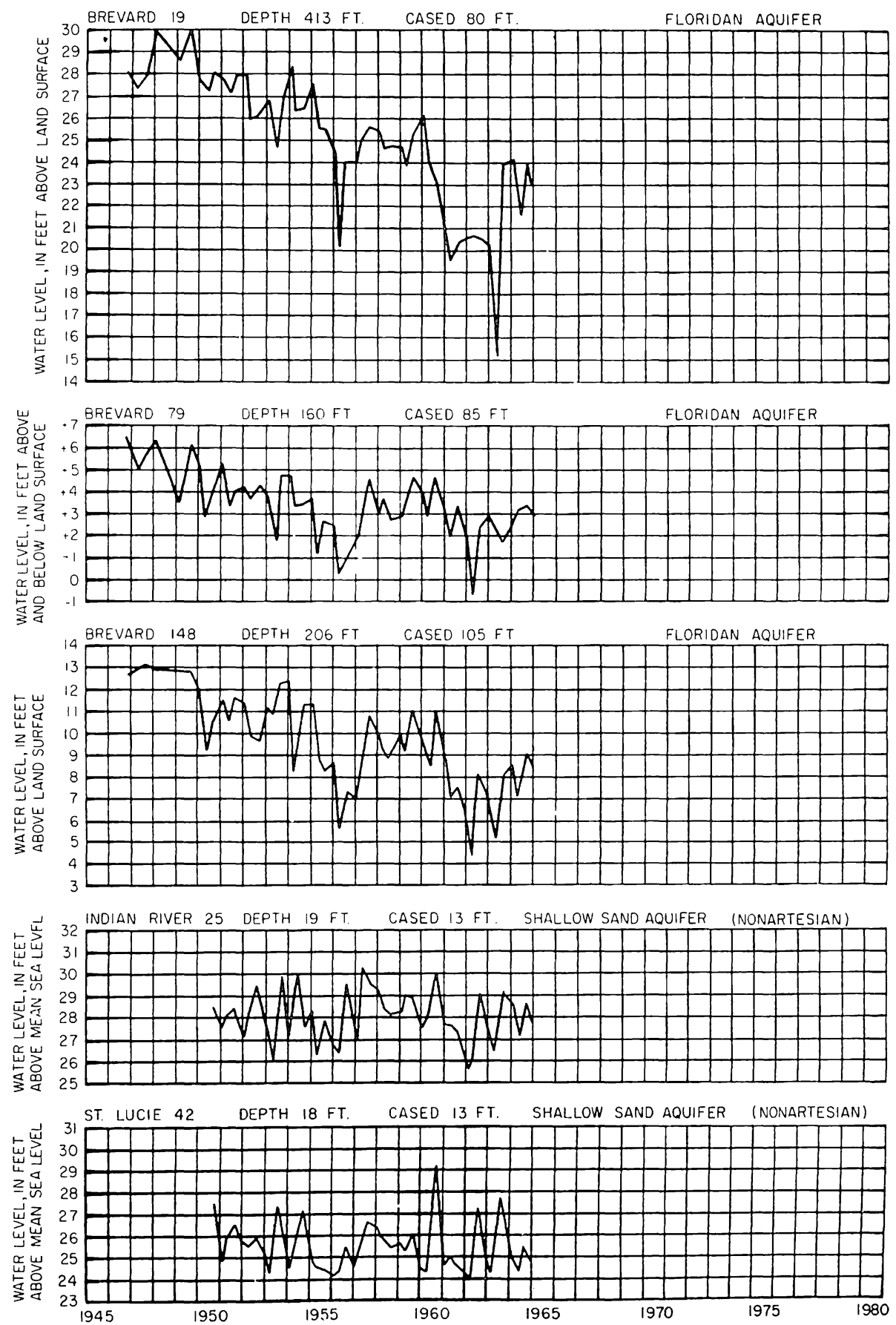

Figure 32. Hydrographs showing trends and fluctuations of water levels near Cape Kennedy and eastern-central coastal Florida. 

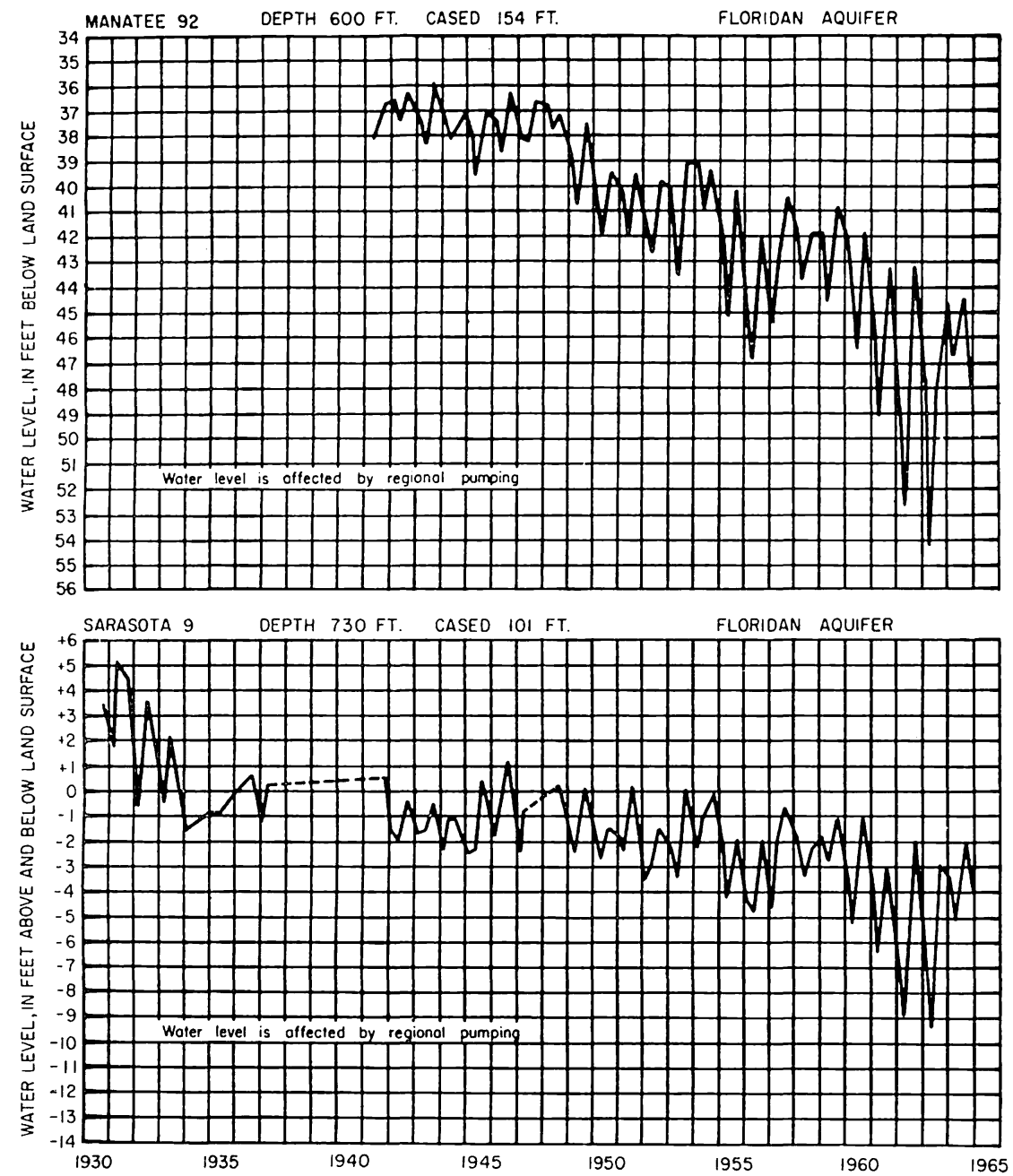

Figure 33. Hydrographs showing trends and fluctuations of water levels in wells Manatee 92 and Sarasota 9, Sarasota-Bradent on area.

both wells show declines of artesian water levels in the Floridan aquifer in Manatee and Sarasota counties. Levels in well Manatee 92 have declined 17.09 feet from 37.10 feet below land surface in May 1947 to a new record low of 54.19 feet below land surface in April 1963. Water levels in this well have been declining at an average overall rate of about one foot each year since 1947. Levels in well Sarasota 9 have declined 8.45 feet from 0.95 foot above land surface in May 1947 to a new record low level of 9.40 feet below land surface in May 1963. Water levels have been declining 


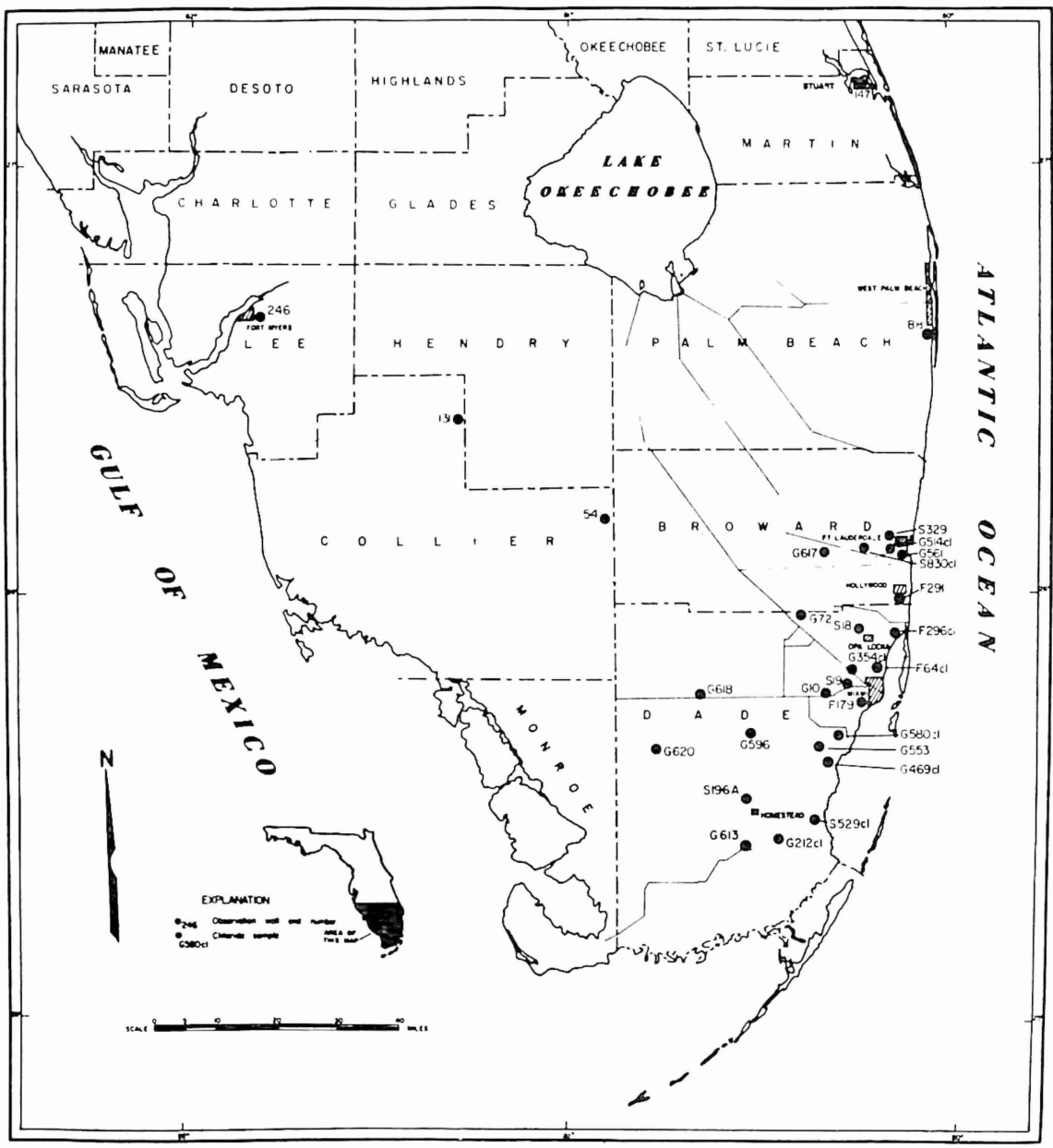

Figure 34. Map showing location of wells in southern Florida for which hydrographs are given.

at an average rate of 0.4 foot per year in well Sarasota 9 since 1931 .

Comparison of the hydrograph of well Manatee 92 to that of well Sarasota 9 shows that the decline is accelerating in Manatee County. The regional extent of the decline is shown by hydrographs of well Hillsborough 30 (fig. 23) and wells Manatee 92 and Sarasota 9 (fig. 33). The decline includes an area of about at least 600 square miles extending from southern Hillsborough County to northern Sarasota County. 

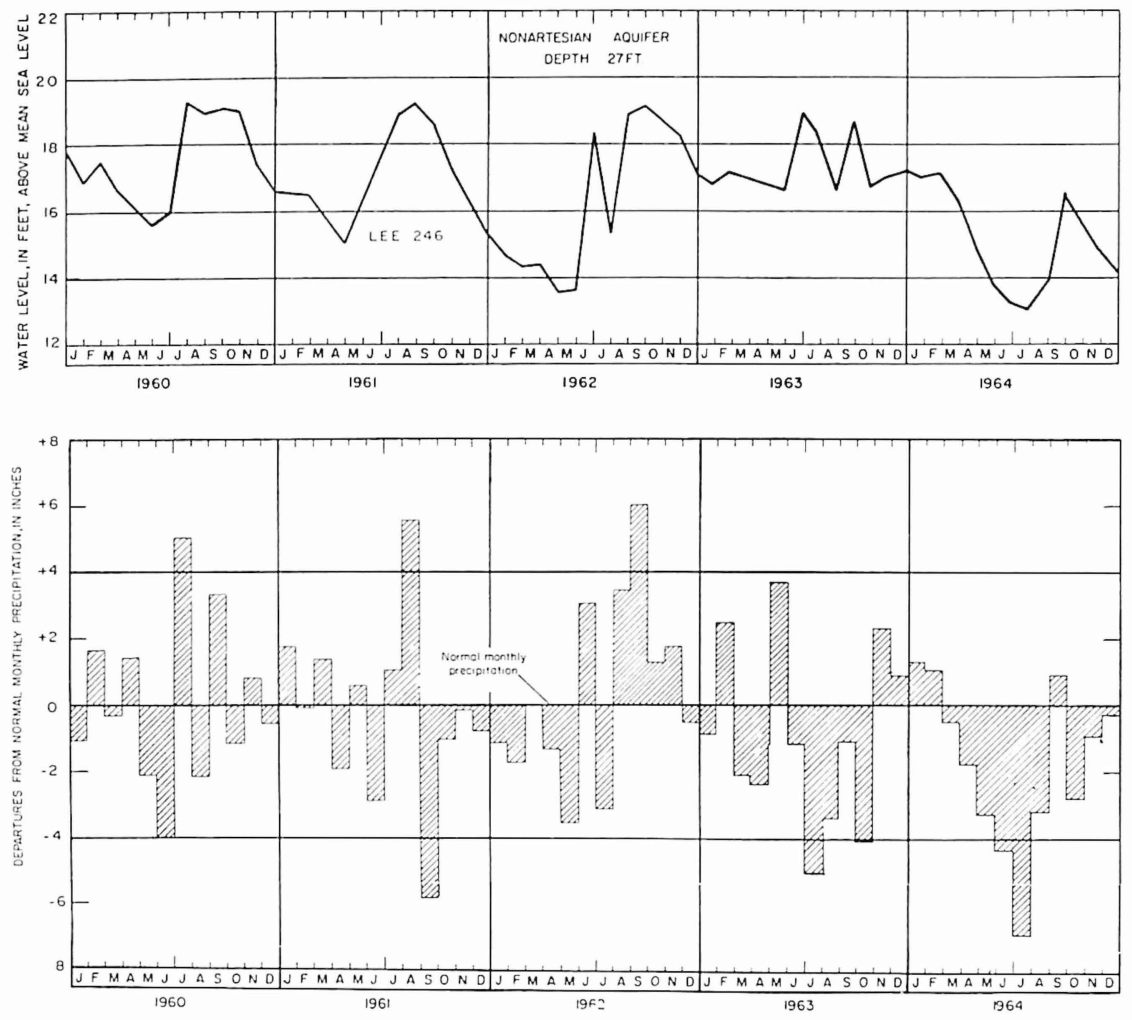

Figure 35. Hydrographs showing trends and fluctuations of water levels in well Lee 246 near Ft. Myers and departures from normal monthly precipitation at Ft. Myers, 1960-64.

\section{SOUTHERN FLORIDA}

The southern Florida region includes all counties south of a line through Desoto County and covers an area of about 17,500 square miles. The region and the locations of selected observation wells for which hydrographs are presented are shown on figure 34 .

In southern Florida, nonartesian aquifers are the principal source of water supply. In the coastal areas of Martin and Palm Beach counties, a nonartesian shallow-sand aquifer is the chief source of supply; in Broward and Dade counties, the Biscayne aquifer is the principal source; and in southwestern coastal Florida and inland areas, nonartesian shallow-sand and shell aquifers are the main sources. 

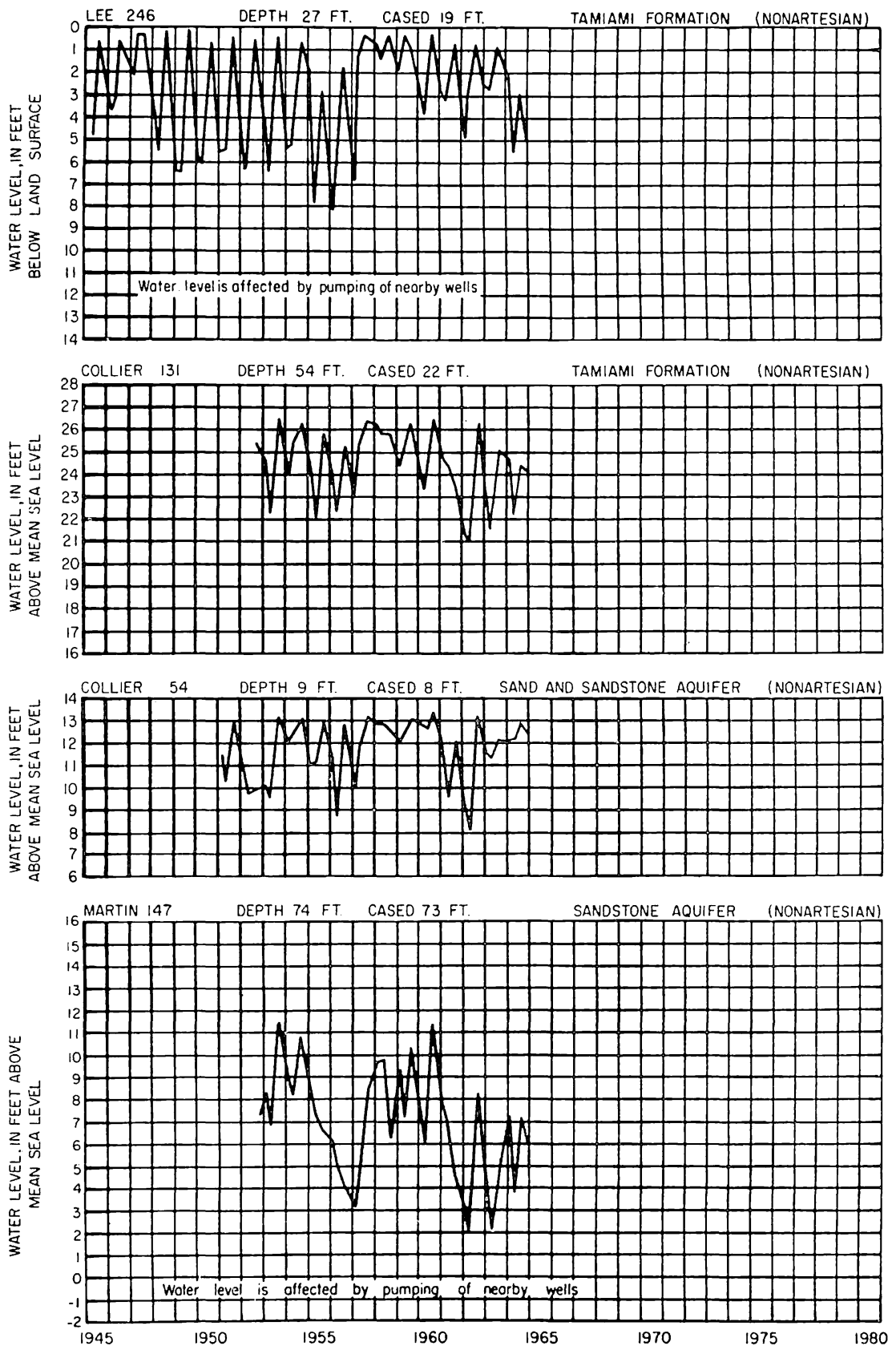

Figure 36. Hydrographs showing trends and fluctuations of water levels in wells Lee 246 near Ft. Myers, Collier 54 Everglades area, Collier 131 near Immokalee, and Martin 147 at Stuart. 


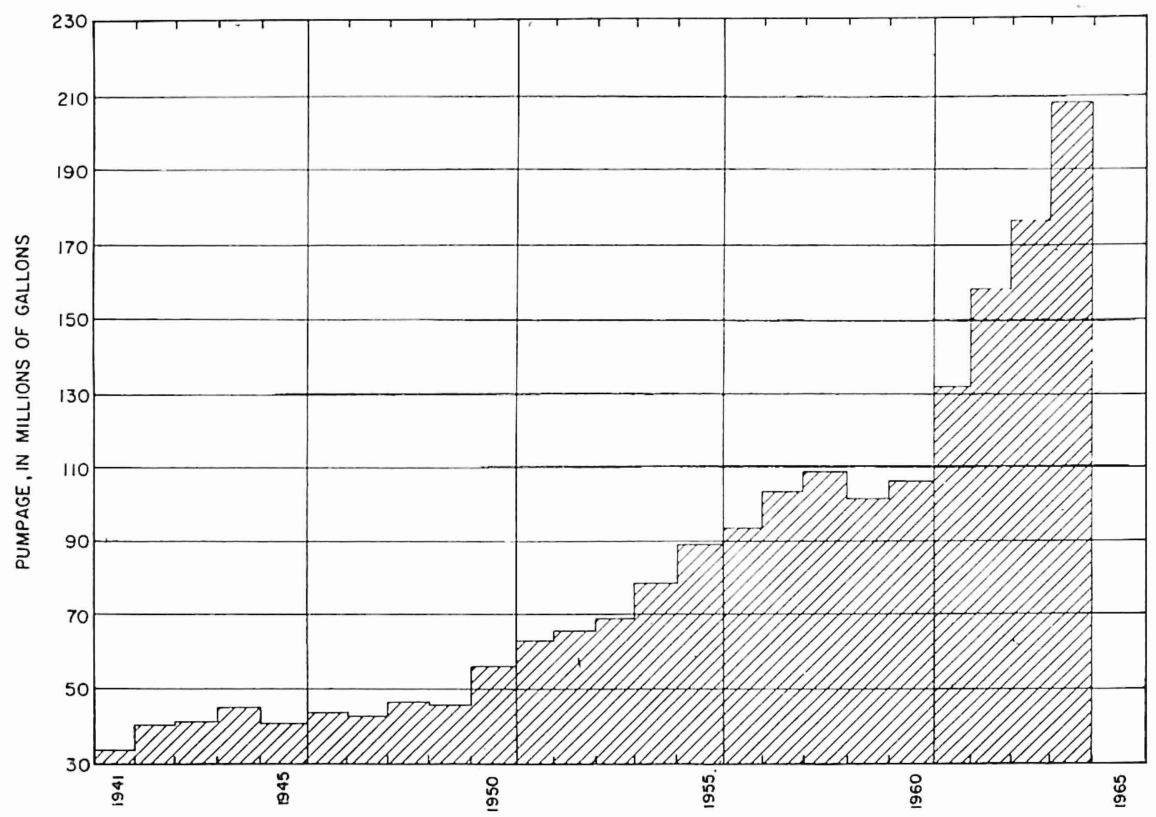

Figure 37. Graph of total year pumpage, City of Stuart.

FT. MYERS AREA

The Ft. Myers area includes Lee and Charlotte counties, and like the Bradenton-Sarasota area to the north is developing rapidly as a winter tourist and retirement center.

The principal source of ground water in the Ft. Myers area is the nonartesian aquifers. Figure 35 shows the seasonal fluctuations of ground-water levels in well Lee 246 and rainfall at Ft. Myers for the period 1960-64. Generally, seasonal fluctuations of water levels in nonartesian aquifers closely correspond to seasonal fluctuations in the amounts of rainfall. Figure 36 shows the trends and fluctuations of water levels in nonartesian aquifers for selected wells in southern Florida.

\section{STUART-WEST PALM BEACH AREA}

This area includes coastal parts of Martin and Palm Beach counties and is a segment of the rapidly growing, populous, coastal complex extending from Jacksonville southward through the Keys. Average yearly municipal pumpage at Stuart increased nearly 450 percent between 1941-45 and 1961-64 as shown in figure 37 .

The principal source of water supply in the Stuart area is the 

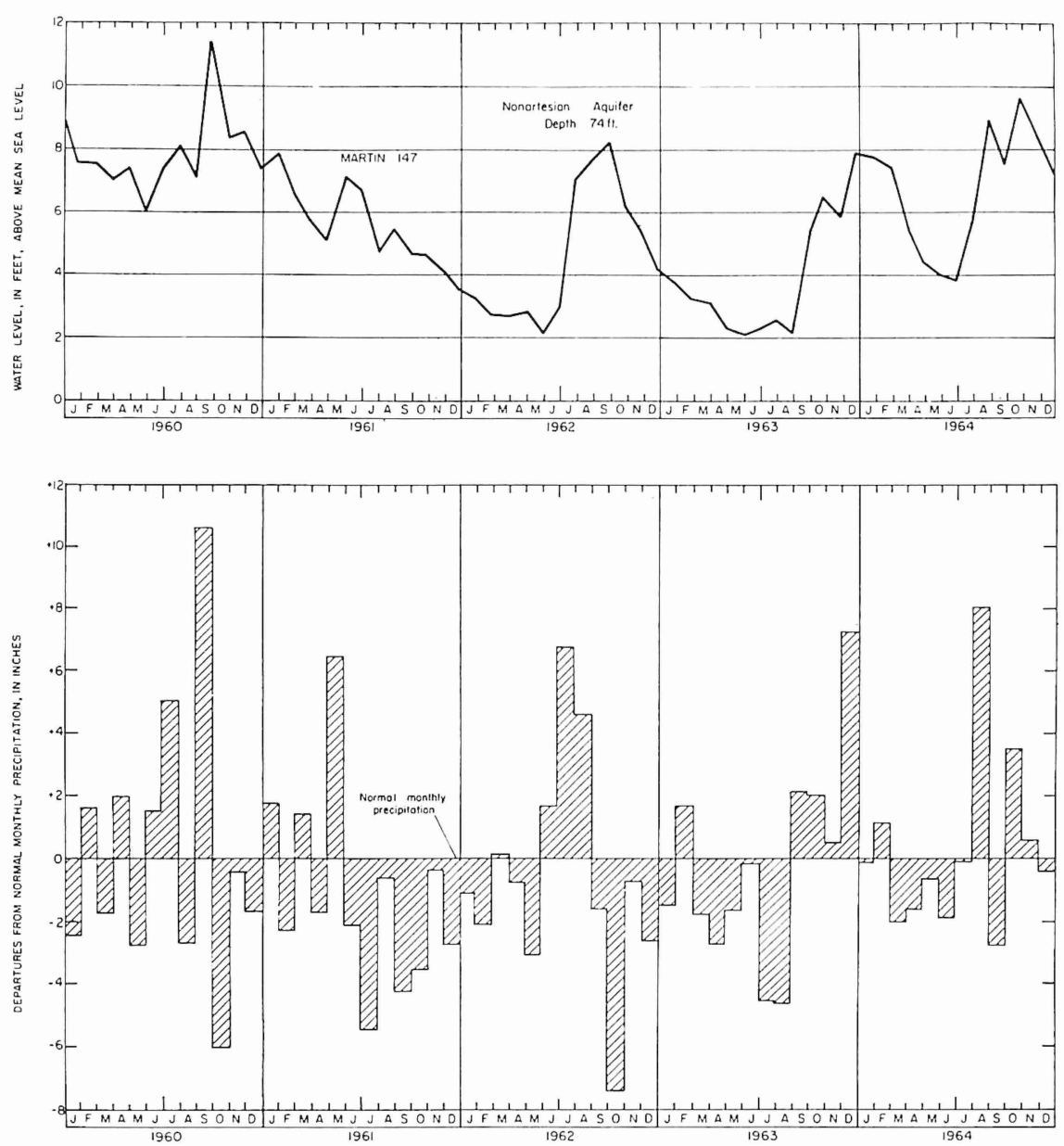

Figure 38. Hydrographs showing trends and fluctuations of water levels in well Martin 147 and departures from monthly normal precipitation at Stuart, 1960-64.

nonartesian shallow-sand aquifer. Trends of water levels in the nonartesian aquifer at Stuart are shown in figure 36 . The hydrograph of well Martin 147 shows a downward trend of nonartesian levels. Levels declined to a record-low level of about 2 feet above mean sea level in the spring of 1962 and 1963. The declines during 1961-63 were caused, in part, by increased pumping in the Stuart well field. Although pumpage increased during 1964, water levels rose in response to above average rainfall. Figure 38 shows trends of water levels and rainfall recorded at Stuart, 1960-64. 

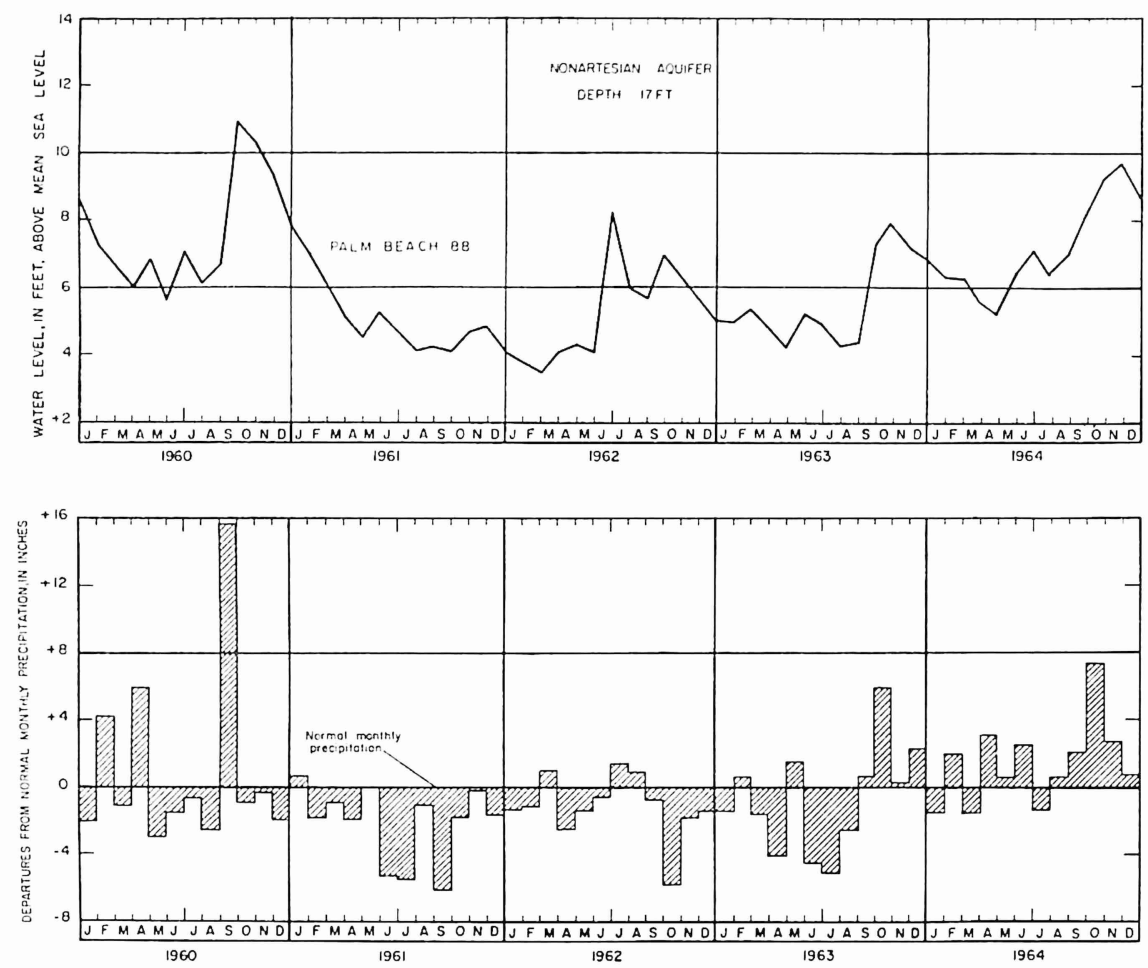

Figure 39. Hydrographs showing trends and fluctuations of water levels in well Palm Beach 88 and departures from monthly normal precipitation at West Palm Beach, 1960-64.

The Biscayne aquifer is the chief source of water supply in southern Palm Beach, Broward, and Dade counties. Figure 39 shows the trends and fluctuations of end-of-month water levels in well Palm Beach 88 and rainfall data at West Palm Beach. Fluctuations of water levels for several selected wells are shown in figures 40 and 41 .

\section{FT. LAUDERDALE AREA}

The Ft. Lauderdale area includes the populous coastal part of Broward County, extending from the Deerfield-Boca Raton area in the northern part of Broward County, to the Hollywood area in the southern part of the county. Long-term downward trends of water levels in the Biscayne aquifer in, and adjacent to, the Ft. Lauderdale area are shown by the hydrograph of well Broward S329 at Ft. Lauderdale (fig. 41).

The Biscayne aquifer contains salty water in areas adjacent 

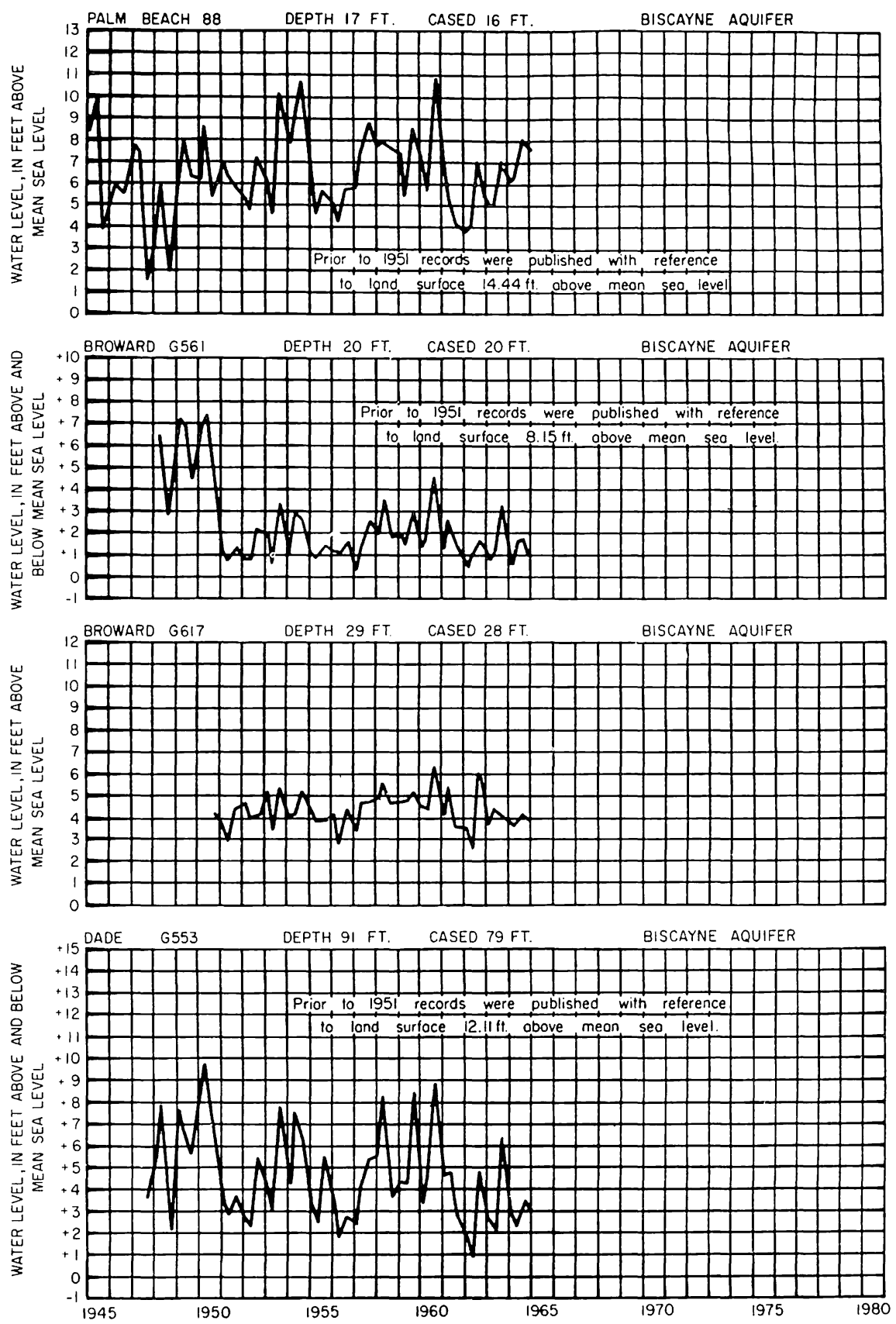

Figure 40. Hydrographs showing trends and fluctuations of water levels in wells Palm Beach 88 near West Palm Beach, Broward G561 and G617 near Ft. Lauderdale, and Dade G553 near Miami. 

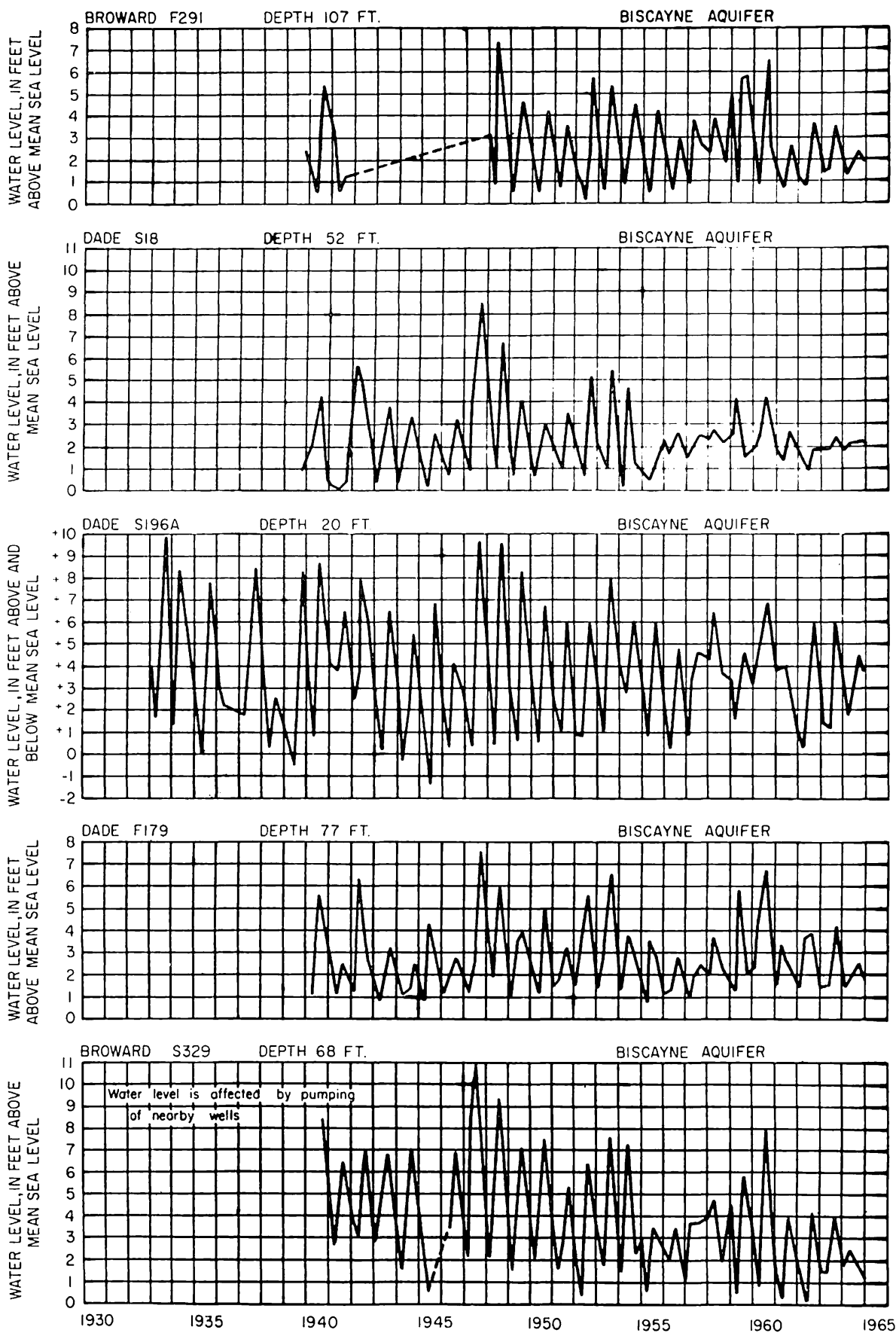

Figure 41. Hydrographs showing trends and fluctuations of water levels in wells Broward F291 at Hollywood, Dade S18 near Miami, Dade S196A near Homestead, Dade F179 at Miami, and Broward S329 near Ft. Lauderdale. 

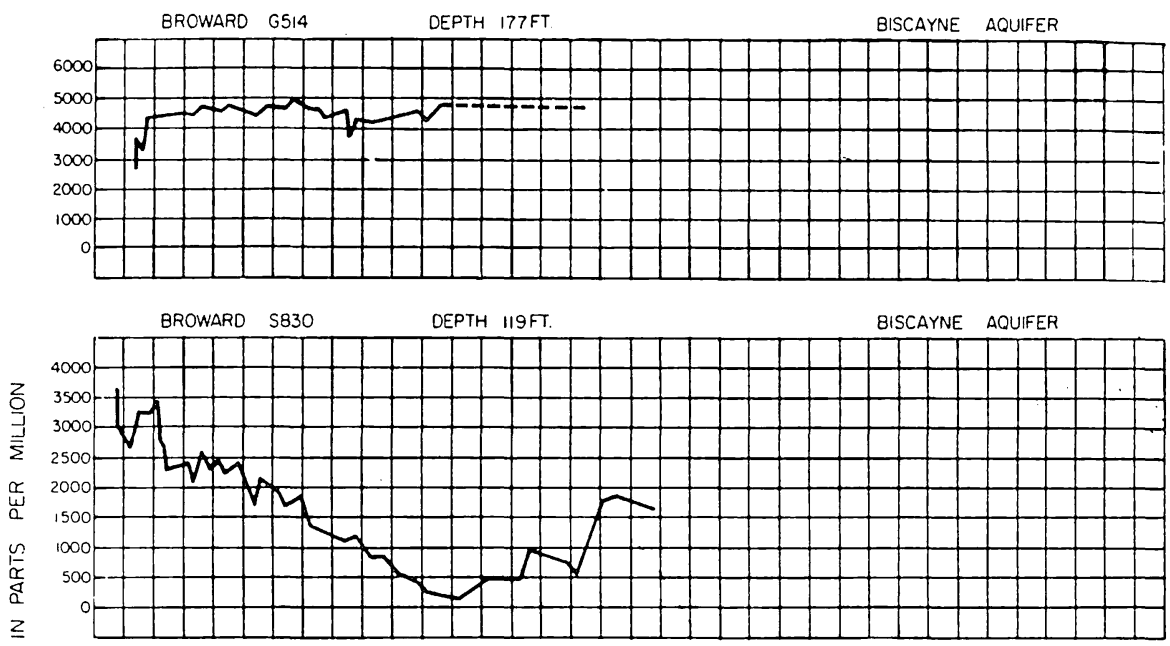

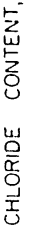
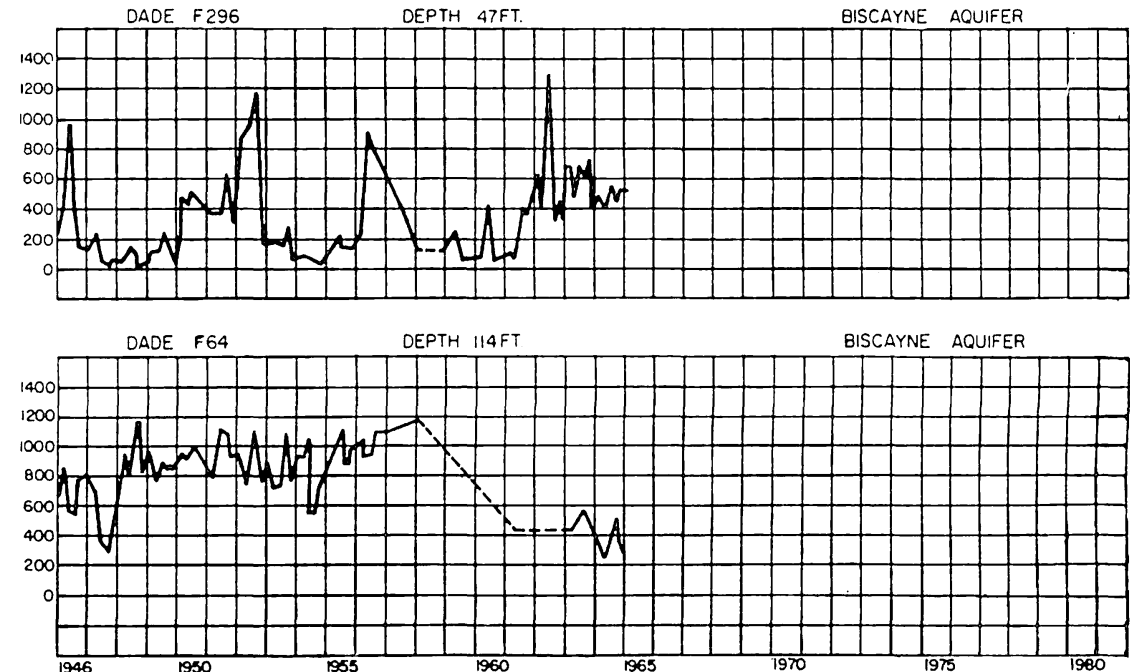

Figure 42. Hydrographs showing changes in chloride content of water in wells Broward G514 and S830 near Ft. Lauderdale, and Dade F296 and F64 near Miami.

to the coast and along tidal canals. Figure 42 shows graphs of the chloride content of water in wells Broward G514 and S830 in the vicinity of the Ft. Lauderdale Dixie well field and in wells Dade F296 and F64 in North Miami Beach and Miami. The chloride content in well Broward S830 declined from about $3700 \mathrm{ppm}$ in 1947 to the lowest chloride content of record in 1958. The chloride content increased from the low of 1958 to nearly $2000 \mathrm{ppm}$ in 1963. 
The Miami area includes Broward and Dade counties and is the most populous area in the State. The principal source of water supply is the Biscayne aquifer, the extent of which is shown on figure 1.

The locations of selected observation wells in the Miami area for which hydrographs are given, are shown by figure 34 .

Water-level observations were made as early as 1933 near Homestead in well Dade S196A. Long-term record of water-level fluctuations at Homestead are shown in figure 41. Figure 43 shows trends of water levels and rainfall recorded at Homestead Experimental Station 1960-64.

Except for the relatively narrow coastal strip, most of the Miami area is occupied by the Everglades. Fluctuations of groundwater levels in the Everglades are shown by hydrographs of wells Dade G72, G596, G618, and G620, figures 44 and 45 .

Fluctuations of ground-water levels in the Biscayne aquifer in the vicinity of Miami are illustrated by hydrographs of wells Dade G10 about 5 miles west of Miami, Dade S19 at Miami Springs (fig. 44), and well Dade F179 at Miami (fig. 41). The water level in well Dade $\mathrm{S} 19$ is affected by pumping in the municipal well field of the City of Miami.

In the Miami area, as in other coastal areas, the presence of salty water in an aquifer is signalled by high chloride content of the ground water. The seaward reaches of the Biscayne Aquifer contain sea water and some encroachment of sea water into the aquifer has occurred through the years. Water control measures have checked the encroachment and as a result a freshening of the ground water has occurred in some areas.

Generally, chloride content of ground water decreased in the Miami area and in southern coastal Dade County during 1963-64. Figure 42 shows fluctuations of chloride content of ground water in the Biscayne aquifer in the Miami area. Chloride content of ground water in well Dade F64 in Miami decreased to the lowest of record since 1947. During 1963-64, chlorides ranged from 250 to $550 \mathrm{ppm}$ in this well. Chloride content of ground water in the Biscayne aquifer ranged from 400 to $700 \mathrm{ppm}$ in well F296 on the coast north of Miami. Chloride content was generally lower during 1963-64 than during 1962. Near the eastern edge of the Miami well field area in Miami Springs, chloride content decreased from 900 

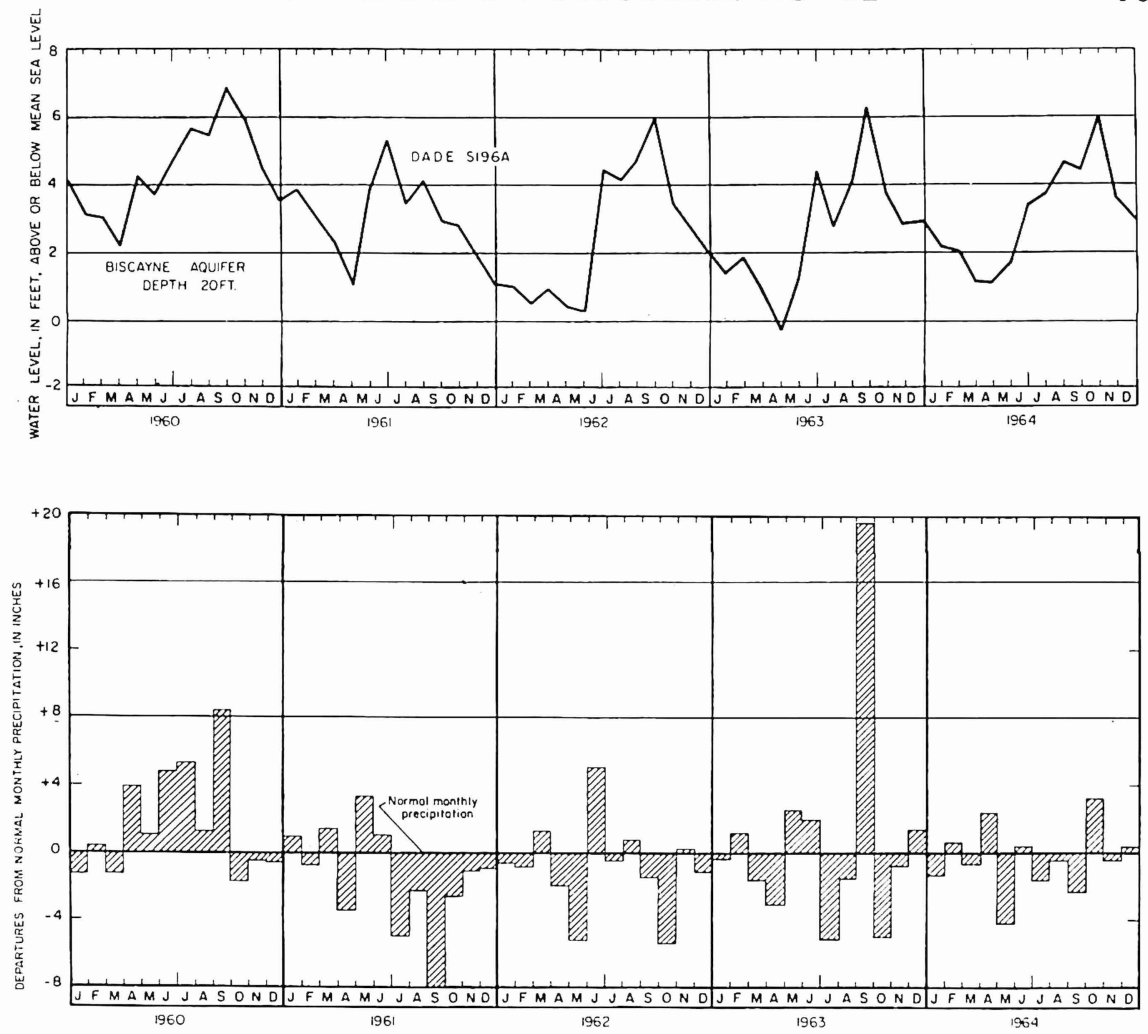

Figure 43. Hydrographs showing trends and fluctuations of water levels in well Dade S196A, and departures from monthly normal precipitation at Homestead Experimental Station, 1960-64.

ppm in 1946 to $100 \mathrm{ppm}$ in 1962 in well Dade G354. During 1963-64, chloride content decreased to the lowest of record in this well.

In southern coastal Dade County, chloride content of ground water in the Biscayne aquifer generally decreased or remained at low concentrations in several areas during 1963-64. Chloride content decreased to less than $500 \mathrm{ppm}$ in well Dade S529 on the coast and that of well Dade G212 southeast of Homestead remained at less than 200 ppm during 1963-64. In sharp contrast, the chloride content in well Dade G469 near the coast south of Miami increased from about $20 \mathrm{ppm}$ in 1961 to about $8600 \mathrm{ppm}$ in 1964 as a result of new canal construction in that area.

In the Miami area, as in other coastal areas, the contamination of the Biscayne aquifer by the encroachment of salty water is an ever-present problem. Through intensive practice of water control the problem of prevention of salt-water contamination has been solved. In many areas where contamination existed the situation 

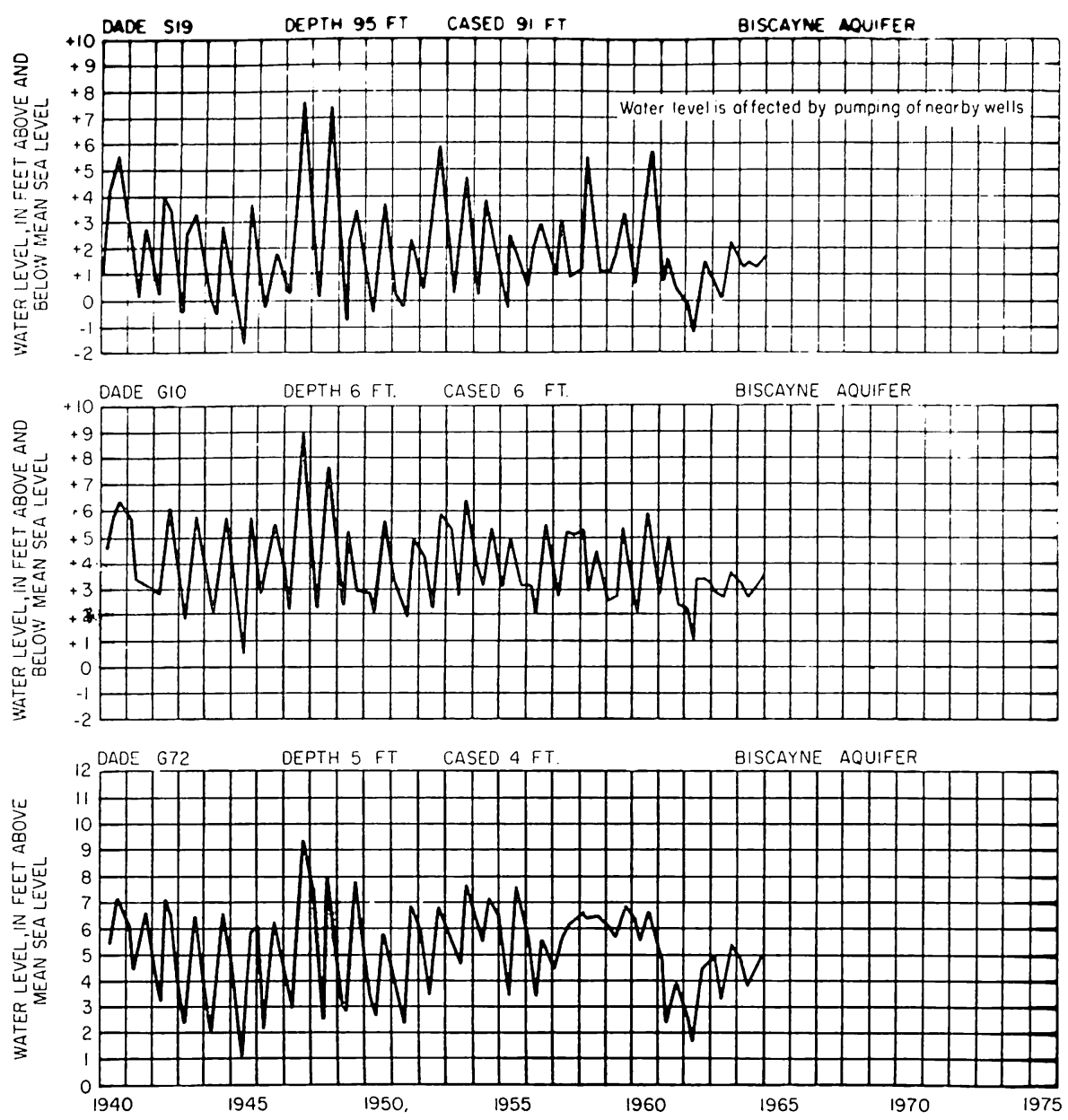

Figure 44. Hydrographs showing trends and fluctuations of water levels in wells Dade S19 and G10 near Miami, and Dade G72 northwest of Opalocka.

has been alleviated by water control. The effectiveness of the method of control is illustrated in figure 46 by the chloride graphs of wells Dade G212, G354, and S529. 

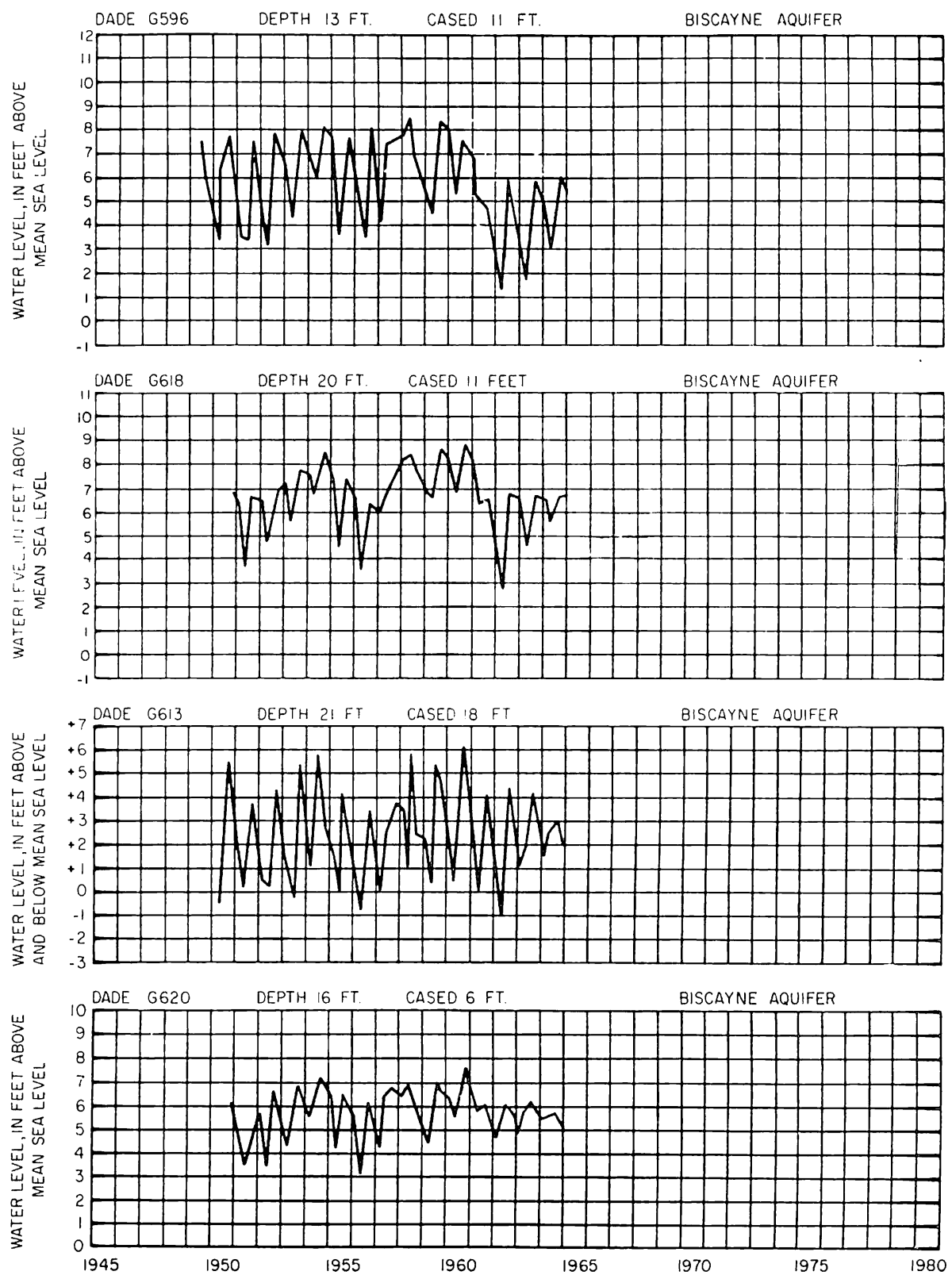

Figure 45. Hydrographs showing trends and fluctuations of water levels in wells Dade G596, G618, G613, and G620 in central Dade County. 

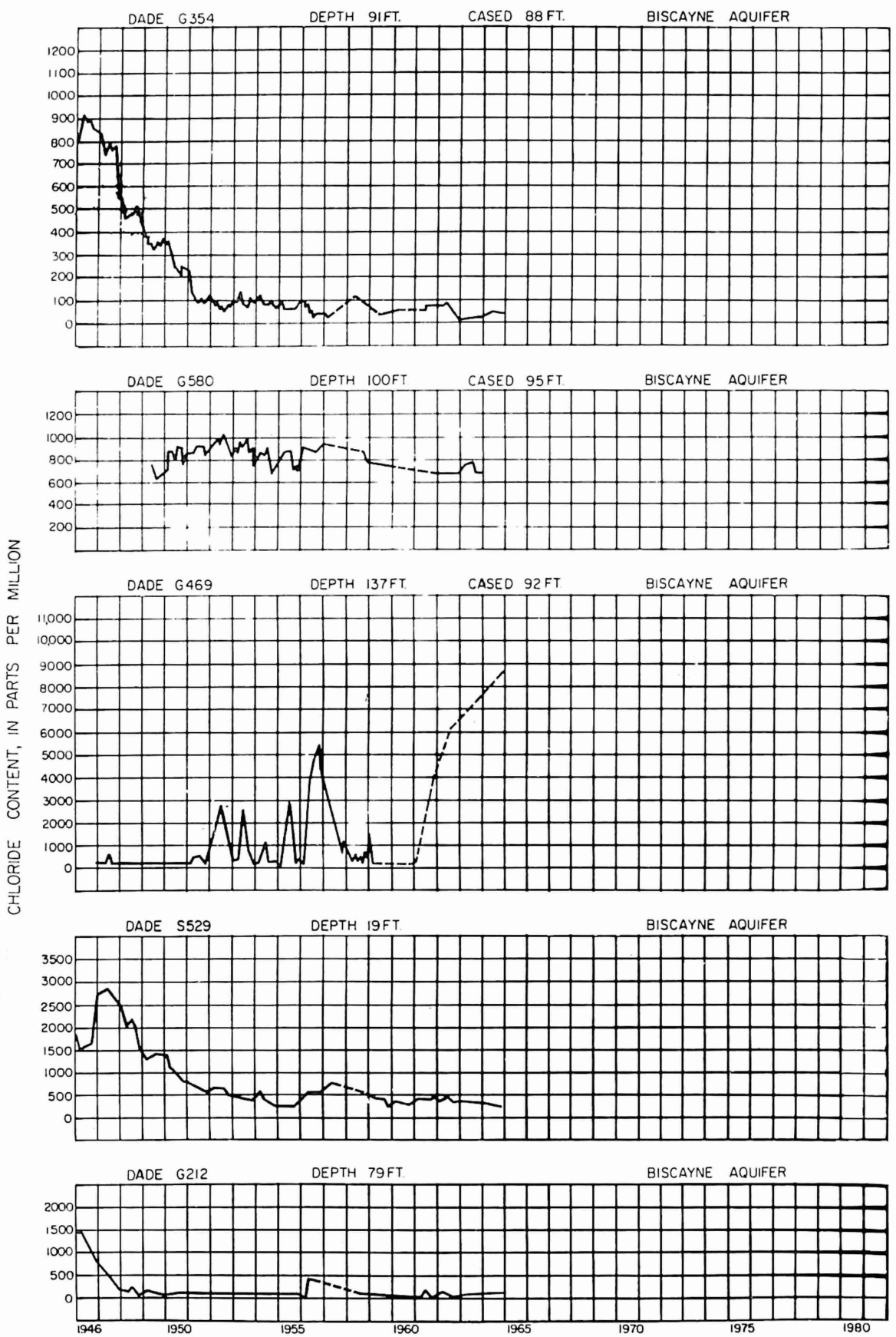

Figure 46. Hydrographs showing changes in chloride content of water in wells Dade G354 and G580 near Miami and Dade G469, S529, and G212 in southeastern Dade County. 




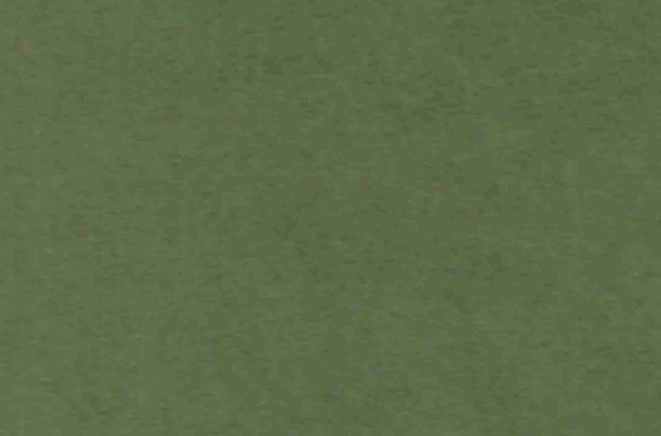

